The submitted manuscript has been created by

the University of Chicago as Operator of

Argonne National Laboratory ("Argonne") under

Contract No. W-31-109-ENG-38 with the U.S.

Department of Energy. The U.S. Government

retains for itself, and others acting on its behalf, a

paid-up, nonexclusive, irrevocable worldwide

license in said article to reproduce, prepare

derivative works, distribute copies to the public,

and perform publicly and display publicly, by or

on behalf of the Government. 


\title{
Letter of Intent
}

\section{to build an Off-axis Detector}

\author{
to study $\nu_{\mu} \rightarrow \nu_{e}$ oscillations
}

with the NuMI Neutrino Beam

Version 6.0

July 17, 2002

\begin{abstract}
Argonne - Athens - Berkeley - Boston - Caltech - Chicago College de France - Fermilab - Harvard - ITEP - Lebedev UC-London - LSU - MIT - MSU - Minnesota-Duluth - Minnesota-Minneapolis TUM-München - NIU - Ohio-Athens - Oxford - Pittsburgh - Princeton Rochester - Rutherford - São Paulo - Stanford - Stony Brook - Sussex Texas-Austin - TMU-Tokyo - Tufts - UCLA - Virginia Tech - York-Toronto
\end{abstract}


D. Ayres, G. Drake, M. Goodman, V. Guarino, T. Joffe-Minor, D Reyna,

R. Talaga, J. Thron

Argonne National Laboratory, IL

N. Giokaris, G. Tzanakos

University of Athens, Athens, Greece

E. Kearns, C. Walter

Boston University, Boston, MA

K. Heeger, K-B. Luk

University of California, Berkeley, CA

B. Choudhary, D. Michael

Caltech, Pasadena, $C A$

E. Blucher

University of Chicago, Chicago, IL

T. Patzak

College de France, Paris, France

D. Carey, S. Childress, J. Cooper, S. Geer, R. Hatcher, D. Harris, J. Hylen, P. Kasper, P. Lucas, V. Makeev, A. Marchionni, N. Mokhov, J .Nelson, A. Para ${ }^{\dagger}$, S. Parke, S. Pordes, R. Ray, P. Shanahan, V. Scarpino, P. Spentzouris

Fermilab, Batavia, IL

G.J. Feldman, A. Lebedev, R. Lee, M.D. Messier, S.-M. Seun

Harvard University, Cambridge, $M A$

I. Trostin

ITEP, Moscow, Rusia

V. Ryabov

Lebedev Institute, Mosow, Russia

J. Thomas

University College London, London, UK

R. Imlay, W. Metcalf, R. Svoboda

Louisiana State University, Baton Rouge, LA 


\author{
K. Scholberg \\ MIT, Cambridge, MA \\ C. Bromberg, R. Richards \\ Michigan State University, East Lansing, MI
}

A. Habig

University of Minnesota, Duluth, MI

P. Border, T. Chase, D. DeMuth, K. Heller, S. Kasahara, M. Marshak, L. Mualem, N. Pearson, E. Peterson, K. Ruddick, J. Urheim University of Minnesota, Minneapolis, MN

P. Huber, M. Lindner, W.Winter

Technische Universität München, München, Germany

C. Albright

Northern Illinois University, DeKalb, IL

C.R. Brune, D.S. Carman, S.M. Grimes, K.H. Hicks, A.K Opper

Ohio University, Athens, $\mathrm{OH}$

G.D. Barr, J.H. Cobb, A. de Santo, N. Tagg, A. Weber, K. Zuber

Oxford University, Oxford, UK

D. Naples, V. Paolone

University of Pittsburgh, Pittsburgh, PA

K. T. McDonald

Princeton University, Princeton, NJ

A. Bodek, H. Budd, P. deBarbaro, G. Ginther, S. Manly, K. McFarland, W. Sakumoto, P. Slattery, M. Zielinski

University of Rochester, Rochester, NY

P. Litchfield, G. Pearce, D. Petyt

Rutherford Appleton Laboratory, Chilton, Didcot, UK

H. Nunokawa

Universidade Estadual de Paulista, São Paulo, Brazil

S. Avvakumov, C. Cueva, G. Irwin, H. Kang, S. Wojcicki

Stanford University, Stanford, CA 
R. Shrock

State University of New York, Stony Brook, NY

P. Harris

University of Sussex, Brighton, UK

S. Kopp, K. Lang

University of Texas, Austin, TX

H. Minakata

Tokyo Metropolitan University, Tokyo, Japan

H. Gallagher, T. Kafka, A. Mann, J. Schneps

Tufts University, Boston, MA

D. Cline, K. Lee

UCLA, Los Angeles, CA

C. Hagner, N. Morgan

Virginia Tech, Blacksburg, VT

S. Menary

York University, Toronto, Canada

$\dagger$ Contact person 


\section{Contents}

$\begin{array}{ll}\text { Executive summary } & 6\end{array}$

1 Motivation $1-8$

2 The Role of the MINOS Experiment $\quad 2-14$

3 Possible Evolution of Neutrino Physics $\quad 3-19$

4 Off-Axis NuMI Neutrino Beam $\quad \mathbf{4 - 2 4}$

4.1 NuMI Off-Axis Neutrino Fluxes . . . . . . . . . . . . . . . . . 4-26

4.2 Backgrounds . . . . . . . . . . . . . . . . . . . . . 4-29

4.3 Antineutrino Beams . . . . . . . . . . . . . . . . . 4-29

4.4 Proton Economics . . . . . . . . . . . . . . . . . 4-32

$5 \quad \nu_{\mu} \rightarrow \nu_{e}$ Oscillation Appearance Experiment $\quad$ 5-34

5.1 Statement of the challenge . . . . . . . . . . . . . 5-34

5.2 Possible experimental approaches . . . . . . . . . . . . 5-35

5.3 Background measurements and estimates; systematics . . . . . . 5-36

5.4 Physics Potential of the NuMI Off-axis Beam . . . . . . . . . 5-38

5.5 Comparison with JHF Phase I . . . . . . . . . . . . 5-41

6 Future Evolution of the Off-Axis Neutrino Program 6-43

$\begin{array}{lll}7 & \text { Detector } & \text { 7-46 }\end{array}$

8 Detector Sites, Physics Considerations $\quad 8-48$

9 Cost and Schedule, Milestones $\quad \mathbf{9 - 5 0}$

9.1 Cost . . . . . . . . . . . . . . . . . 9-50

9.2 Schedule . . . . . . . . . . . . . . . . . . 9-51

9.3 Milestones . . . . . . . . . . . . . . . . . . . 9-51

References . . . . . . . . . . . . . . . . . 9-54 
A Possible Detector Technologies A-1

A.1 Low Z Tracking Calorimeter . . . . . . . . . . . . . . A-1

A.1.1 Low energy neutrino interactions in low $\mathrm{Z}$ calorimeter . . A-2

A.1.2 Electron identification . . . . . . . . . . . . A-2

A.1.3 Energy resolution . . . . . . . . . . . . . . A A-6

A.1.4 A specific detector example: LoDen - LOw DENsity calorimeter . . . . . . . . . . . . . A

A.1.5 Glass RPC Chambers . . . . . . . . . . . . . . A-9

A.1.6 Readout Electronics . . . . . . . . . . . . . . . A-11

A.1.7 Ancillary Systems . . . . . . . . . . . . . . . A-15

A.1.7.1 Gas System . . . . . . . . . . . A-15

A.1.7.2 HV . . . . . . . . . . A A-16

A.1.8 Modular Detector Design . . . . . . . . . . . . A-17

A.1.9 Absorber Construction and Composition . . . . . . . . . A-19

A.1.9.1 Containers and Loose Fill Materials . . . . . . . A-19

A.1.9.2 Molded Particleboard . . . . . . . . . . . . A-20

A.1.9.3 External Framework and Self Supporting Struc-

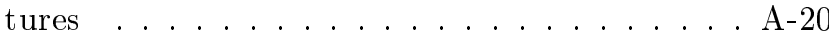

A.1.10 Liquid Scintillator Option . . . . . . . . . . . . A-22

A.1.10.1 Support Structure . . . . . . . . . . . . A-22

A.1.10.2 Signal Collection . . . . . . . . . . . . . A-23

A.1.10.3 Support . . . . . . . . . . . . . . . A-23

A.1.10.4 Readout . . . . . . . . . . . . . . A-23

A.1.10.5 Cost Estimate . . . . . . . . . . . . A-24

A.1.11 Backgrounds in a surface detector . . . . . . . . A-25

A.1.11.1 Cosmic ray rates in the detector . . . . . . A A-25

A.1.11.2 Tertiary particle production by muons interacting in the environment outside the detector . . . A-25

A.1.11.3 Particle production by unseen muons inside the detector . . . . . . . . . . . A-26

A.1.11.4 Gamma rays . . . . . . . . . . . . A-26

A.1.11.5 Measurement of cosmic ray backgrounds . . . . A-27

A.2 Water Cherenkov option . . . . . . . . . . . A-28

A.3 Liquid Argon TPC . . . . . . . . . . . . . . . . . A-33

B Possible Sites for a NuMI Off-Axis Detector B-40

B.1 Criteria for Site Selection . . . . . . . . . . . . . B-40

B.2 Potential Sites . . . . . . . . . . . . . . B-41

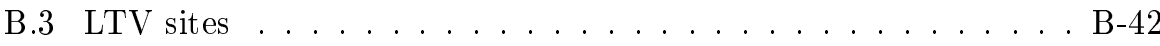


I apologize for this letter being so long but I had no time to make it shorter. Blaise Pascal

\section{Executive summary}

The question of neutrino masses is of fundamental importance. Neutrino oscillations seem to be the only tool available to us to unravel the pattern of neutrino masses and, perhaps, shed some light on the origin of masses in general.

The NuMI neutrino beam line and the MINOS experiment represent a major investment of US High Energy Physics in the area of neutrino physics. The forthcoming results could decisively establish neutrino oscillations as the underlying physics mechanism for the atmospheric $\nu_{\mu}$ deficit and provide a precise measurement of the corresponding oscillation parameters, $\Delta m_{32}^{2}$ and $\sin ^{2} 2 \theta_{23}$.

This, however, is just a beginning of a long journey into uncharted territories. The key to these new territories is the detection of $\nu_{\mu} \rightarrow \nu_{e}$ oscillations associated with the atmospheric $\nu_{\mu}$ deficit, controlled by the little known mixing angle $\sin ^{2} 2 \theta_{13}$. A precise measurement of the amplitude of these oscillations will enable a determination of the pattern of the mass hierarchy of neutrinos. If the solar neutrino experiments determine that the value $\Delta m_{12}^{2}$ is in the range of $10^{-5}-10^{-4} \mathrm{eV}^{2}$ then the measurement of the $\mathrm{CP}$ violation in the neutrino sector may well be within our reach.

The full potential of the NuMI neutrino beam can be exploited by complementing the MINOS detector, under construction, with a new detector(s) placed at some off-axis position and collecting data in parallel with MINOS. The first phase of the proposed program includes a new detector, optimized for $\nu_{e}$ detection, with a fiducial mass of the order of $20 \mathrm{kton}$ and exposed to neutrino and

antineutrino beams. In a five year run its sensitivity to the $\nu_{\mu} \rightarrow \nu_{e}$ oscillations will be at least a factor of ten beyond the current limit.

The future direction of the program will depend on the results of this first phase, but it is very likely that it will be a combination of a significant increase of the neutrino beam intensity via an upgraded proton source and an increase of the detector mass by a factor of five or so. Depending on the circumstances, the goals of Phase II may be a further increase of the sensitivity of a search for $\nu_{\mu} \rightarrow \nu_{e}$ oscillations, or, perhaps, a measurement of the CP violating phase $\delta$ in the lepton sector. 
This document is organized as follows:

- Chapter 1 summarizes the physics motivation for the proposed experiment

- Chapter 2 briefly reviews the role of the MINOS experiment in the investigations of neutrino oscillations

- Chapter 3 outlines the possible evolution of the neutrino program in the US and world-wide

- Chapter 4 discusses the properties of the NuMI neutrino beam, especially at the off-axis positions

- Chapter 5 discusses experimental considerations for a $\nu_{\mu} \rightarrow \nu_{e}$ oscillation experiment

- Chapter 6 discusses possible detectors. Details of specific detector designs and various technical aspects are described in the Appendix A.

- Chapter 7 addresses the issues of possible sites for the new experiment. Details of a specific site: the LTV mine are described in the Appendix B.

- Chapter 8 discusses possible scenarios of the evolution of the neutrino program at Fermilab

- Chapter 9 outlines a possible schedule and milestones 


\section{Chapter 1}

\section{Motivation}

The SuperKamiokande[1] experiment has recently provided very strong evidence that the muon neutrino undergoes flavor changing transitions. These transitions are seen for neutrinos whose path length divided by energy $(\mathrm{L} / \mathrm{E})$ is greater than $\sim 10^{2} \mathrm{~km} / \mathrm{GeV}$. SuperK also has some supporting evidence that these muon neutrinos are transformed primarily into tau neutrinos. Although the SuperKamiokande detector has some sensitivity to flavor transitions of electron neutrinos their data provides no evidence that electron neutrinos are involved in these transitions. In fact, the Chooz [2] reactor experiment provides a tighter constraint on the upper limit on the probability of electron neutrino flavor transitions of $5 \%$, at the values of L/E for which SuperKamiokande sees muon neutrino flavor transitions. This leaves open the interesting and important question: What is the role of the electron neutrino in flavor transitions at these values of L/E? A measurement or stringent limit on the probability of $\nu_{\mu} \rightarrow \nu_{e}$ for such values of $\mathrm{L} / \mathrm{E}\left(10^{2}\right.$ to $\left.10^{3} \mathrm{~km} / \mathrm{GeV}\right)$ is an important step in understand these neutrino flavor transitions in atmospheric neutrinos. As the NuMI beam is primarily a $\nu_{\mu}$ beam, the observation of $\nu_{e}$ appearance would address this question directly. This is the primary goal of the experiment described by this letter of intent.

The SNO[3] experiment has recently reported large transitions of solar electron neutrinos to muon and/or tau neutrinos. The onset of these transitions occurs for an $\mathrm{L} / \mathrm{E}$ which is a least one order of magnitude larger than the $\mathrm{L} / \mathrm{E}$ for which SuperKamiokande sees flavor transitions in atmospheric muon neutrinos. If these transitions occur near the lower allowed values of $\mathrm{L} / \mathrm{E}$ then the transition probability $\nu_{\mu} \rightarrow \nu_{e}$ measured by an experiment in the NuMI beam can have some sensitivity to the flavor transitions associated with solar neutrinos.

The LSND[4] experiment has reported small muon antineutrino to electron antineutrino transitions for values of $\mathrm{L} / \mathrm{E}$ which are less than two orders of magnitude smaller than the transitions seen in atmospheric neutrinos. However this transition probability is very small, compared to that observed in atmospheric and solar neutrinos, on the order of $0.3 \%$. If this result is confirmed by the up 
coming mini-Boone [5] experiment, this could be an important background for a measurement of $\nu_{\mu} \rightarrow \nu_{e}$ transitions at the larger values of $\mathrm{L} / \mathrm{E}$ associated with atmospheric neutrinos.

To explain the above phenomena, extensions to the Standard Model are required. The simplest and most widely accepted extension is to allow the neutrinos to have masses and mixings such that the above phenomena are explained by neutrino oscillations. The masses and mixing of the neutrinos in these extensions would be the low energy remnant of some yet to be determined high energy physics. Thus, neutrino masses and mixing provide a unique window on physics that is inaccessible to current or near future collider experiments. One popular theory is the so called "seesaw" scenario where the active left handed neutrinos seesaw off their heavier right handed (sterile) partners leaving three very light Majorana neutrinos. It is already clear that the masses and mixings in the neutrino sector are very different than the masses and mixing in the quark sector and that a detailed understanding of the neutrino masses and mixings will be important in differentiating fermion mass theories. Also, they may provide the key to advancing our theoretical understanding of this fundamental question.

If the neutrinos have masses and mixings then the neutrino mass eigenstates, $\nu_{i}=\left(\nu_{1}, \nu_{2}, \nu_{3}, \ldots\right)$ with masses $m_{i}=\left(m_{1}, m_{2}, m_{3}, \ldots\right)$ are related to the flavor eigenstates, $\nu_{\alpha}=\left(\nu_{e}, \nu_{\mu}, \nu_{\tau}, \ldots\right)$ by a matrix $U_{\nu}$,

$$
\left|\nu_{\alpha}\right\rangle=\sum_{i}\left(U_{\nu}\right)_{\alpha i}\left|\nu_{i}\right\rangle
$$

The charged weak current is given by

$$
J_{\lambda}=\bar{\nu}_{L} U \gamma_{\lambda} \ell_{L}
$$

where $\ell=(e, \mu, \tau)$ is the vector of charged lepton mass eigenstates and $U=$ $U_{\nu} U_{\ell}^{\dagger}$ with $U_{\ell}$ being the mixing matrix for chargad leptons in analogy with Eq. 1.1. In the absence of light sterile neutrinos, the $3 \times 3$ lepton mixing matrix $U$ is unitary. Lepton flavor mixing was first discussed (for the $2 \times 2$ case) by Maki, Nakagawa, and Sakata.

If we restrict the light neutrino sector to the three known active flavors and set aside the LSND results ${ }^{1}$ then the unitary matrix MNS matrix, $U$, can be written as

$$
U_{\alpha i}=\left(\begin{array}{ccc}
c_{13} c_{12} & c_{13} s_{12} & s_{13} e^{-i \delta} \\
-c_{23} s_{12}-s_{13} s_{23} c_{12} e^{i \delta} & c_{23} c_{12}-s_{13} s_{23} s_{12} e^{i \delta} & c_{13} s_{23} \\
s_{23} s_{12}-s_{13} c_{23} c_{12} e^{i \delta} & -s_{23} c_{12}-s_{13} c_{23} s_{12} e^{i \delta} & c_{13} c_{23}
\end{array}\right)
$$

where $c_{j k} \equiv \cos \theta_{j k}$ and $s_{j k} \equiv \sin \theta_{j k}$.

\footnotetext{
${ }^{1}$ In the $3+1$ neutrino mass hierarchy the LSND result can be accommodated as a perturbation on the pure active 3 neutrino hierarchy. The $2+2$ mass hierarchy would require major modifications.
} 
With this labeling the atmospheric neutrinos oscillations are primarily determined by $\theta_{23}$ and $\Delta m_{32}^{2}$ whereas the solar neutrino oscillations depend on $\theta_{12}$ and $\Delta m_{21}^{2}$, where $\Delta m_{i j}^{2}=m_{i}^{2}-m_{j}^{2}$. From SuperKamiokande we already have some knowledge of $\left|\Delta m_{32}^{2}\right|=(1.4-4.0) \times 10^{-3} \mathrm{eV}^{2}$ and $\theta_{23} \approx \pi / 4$. SNO and the other solar neutrino experiments indicate $\Delta m_{21}^{2}=+(2-10) \times 10^{-5} \mathrm{eV}^{2}$ and $\theta_{12} \approx \pi / 6$. Furthermore, Chooz (and SuperK) provide us with a limit on $\sin ^{2}\left(2 \theta_{13}\right)$. The Chooz limit is dependent on the input value used for $\left|\Delta m_{a t m}^{2}\right|$; for the current central value $2.5 \times 10^{-3} \mathrm{eV}^{2}$, this is $\sin ^{2}\left(2 \theta_{13}\right)<0.11$, while for $\left|\Delta m_{a t m}^{2}\right|=2.0 \times 10^{-3} \mathrm{eV}^{2}$, it is $\sin ^{2}\left(2 \theta_{13}\right)<0.18$ [2]. Thus the proposed long-baseline neutrino oscillation experiment to search for $\nu_{\mu} \rightarrow \nu_{e}$ will be able to search over a substantial range below this upper bound.

The appearance probability of $\nu_{e}$ in a $\nu_{\mu}$ beam in vacuum is given, to leading order, by

$$
P_{v a c}\left(\nu_{\mu} \rightarrow \nu_{e}\right)=\sin ^{2} \theta_{23} \sin ^{2} 2 \theta_{13} \sin ^{2} \Delta_{a t m}
$$

where $\Delta_{a t m} \approx 1.27\left(\frac{\Delta m_{32}^{2} L}{E}\right)$. If the experiment is performed at the peak of this probability, that is, when $\Delta_{a t m}=\frac{\pi}{2}+n \pi$, then

$$
P_{v a c}\left(\nu_{\mu} \rightarrow \nu_{e}\right) \approx \frac{1}{2} \sin ^{2} 2 \theta_{13} .
$$

The first peak occurs at neutrino energy,

$$
E=1.8 \mathrm{GeV}\left(\frac{\Delta m_{32}^{2}}{3 \times 10^{-3} \mathrm{eV}^{2}}\right)\left(\frac{L}{732 \mathrm{~km}}\right) .
$$

Using the constraint from Chooz of $\sin ^{2} 2 \theta_{13}<0.1$ gives a maximum appearance probability of $5 \%$. To be effective any $\nu_{e}$ appearance experiment has to aim to exclude or convincingly see a signal at least an order of magnitude below this $5 \%$ limit.

The neutrinos in the NuMI beam propagate through the Earth and matter induced contribution to the propagation amplitude are non-negligible. These matter effects have opposite sign for neutrinos and anti-neutrinos and for the normal versus inverted neutrino mass hierarchies. The matter effects can be thus used to distinguish the two possible three neutrino mass hierarchies, see Fig. 1.1. If the experiment is performed at the first peak in the oscillation, as above, the matter effects are primarily a function of the energy of the neutrino beam and the transition probability in matter can be approximated by

$$
P_{\text {mat }}\left(\nu_{\mu} \rightarrow \nu_{e}\right) \approx\left(1 \pm 2 \frac{E}{E_{R}}\right) P_{v a c}\left(\nu_{\mu} \rightarrow \nu_{e}\right)
$$

where $E_{R}$ is the matter resonance energy associated with the atmospheric $\Delta m^{2}$, that is

$$
E_{R}=\frac{\Delta m_{32}^{2}}{2 \sqrt{2} G_{F} N_{e}}=13 \mathrm{GeV}\left(\frac{\Delta \mathrm{m}_{32}^{2}}{3 \times 10^{-3} \mathrm{eV}^{2}}\right)\left(\frac{1.5 \mathrm{gcm}^{-3}}{\mathrm{Y}_{\mathrm{e}} \rho}\right) .
$$




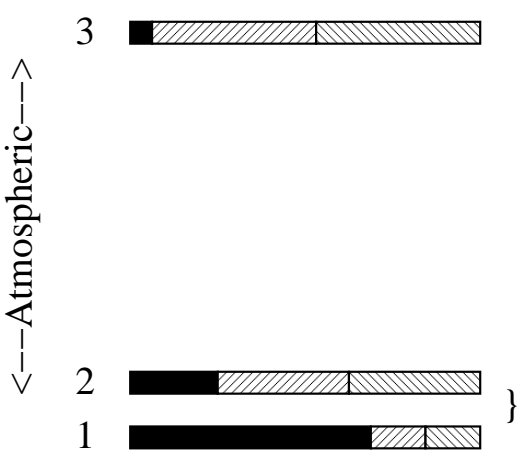

NORMAL

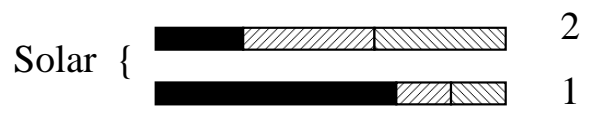

\section{INVERTED}

Figure 1.1: The two allowed three-neutrino mass squared spectrums that account for the oscillations of solar and atmospheric neutrinos. The normal spectrum has $\Delta m_{32}^{2}>0$ and the inverted has $\Delta m_{32}^{2}<0$. The $\nu_{e}$ fraction of each mass eigenstate is indicated by the solid region whereas the $\nu_{\mu}\left(\nu_{\tau}\right)$ fraction is indicated by the right-leaning (left-leaning) hatching. The $\nu_{e}$ fraction in the mass eigenstate labeled, 3 , has been enhanced for clarity.

where $N_{e}$ is the electron number density in the earth, $\rho$ is the matter density and $Y_{e}$ is the electron fraction.

For the normal hierarchy, matter effects enhance (suppress) the transition probability for neutrinos (anti-neutrinos) and vice versa for the inverted hierarchy. For a $2 \mathrm{GeV}$ neutrino energy, matter effects give a $30 \%$ enhancement or suppression in the transition probability.

If the solution to the solar neutrino puzzle is determined to be neutrino oscillations with the parameters in the "Large Mixing Angle" (LMA) region then the $\nu_{\mu} \rightarrow \nu_{e}$ transition probability is sensitive to sub-leading effects and in particular to the $\mathrm{CP}$ violating phase $\delta$.

In vacuum the shift in the transition probability associated with the $\mathrm{CP}$ violating phase is given by

$$
\Delta P_{\delta}\left(\nu_{\mu} \rightarrow \nu_{e}\right) \approx \frac{1}{2} \cos \delta J_{r} \Delta_{\odot} \sin 2 \Delta_{a t m} \pm \sin \delta J_{r} \Delta_{\odot} \sin ^{2} \Delta_{a t m}
$$

where the plus (minus) sign is for neutrinos (anti-neutrinos), $J_{r}=\sin 2 \theta_{12} \sin 2 \theta_{23} \sin 2 \theta_{13} \cos \theta_{13}$ and

$$
\Delta_{\odot}=1.27 \frac{\delta m_{\odot}^{2} L}{E}=\frac{\delta m_{\odot}^{2}}{\delta m_{a t m}^{2}} \Delta_{a t m} \approx \frac{1}{60} \Delta_{a t m} .
$$

In the maximum of oscillations at the atmospheric $\Delta m^{2}$ scale the shift in the 
transition probability dependent on $\delta$ is of order

$$
\left|\Delta P_{\delta}\left(\nu_{\mu} \rightarrow \nu_{e}\right)\right| \sim 0.5 \% \sqrt{\frac{\sin ^{2} 2 \theta_{13}}{0.05}}\left(\frac{\delta m_{\odot}^{2} \sin 2 \theta_{12}}{5 \times 10^{-5} e V^{2}}\right) .
$$

This shift is smaller but of similar size to the shift associated with matter effects.

The full transition probability, in vacuum, is then given by [6]:

$$
\begin{aligned}
P\left(\nu_{\mu} \rightarrow \nu_{e}\right) & =2 \sin \left(2 \theta_{13}\right) s_{23} c_{13} s_{12}\left(s_{12} s_{23} s_{13}-c_{12} c_{23} c_{\delta}\right) \sin ^{2} \phi_{32}+ \\
& +2 \sin \left(2 \theta_{13}\right) s_{23} c_{13} c_{12}\left(c_{12} s_{23} s_{13}+s_{12} c_{23} c_{\delta}\right) \sin ^{2} \phi_{31}- \\
& -2 \sin \left(2 \theta_{12}\right) c_{13}^{2}\left[s_{12} c_{12}\left(s_{13}^{2} s_{23}^{2}-c_{23}^{2}\right)+s_{13} s_{23} c_{23}\left(s_{12}^{2}-c_{12}^{2}\right) c_{\delta}\right] \sin ^{2} \phi_{21} \\
& +\frac{1}{2} \sin \left(2 \theta_{12}\right) \sin \left(2 \theta_{13}\right) \sin \left(2 \theta_{23}\right) c_{13} s_{\delta}\left[\sin \phi_{32} \cos \phi_{32}\right. \\
& \left.-\sin \phi_{31} \cos \phi_{31}+\sin \phi_{21} \cos \phi_{21}\right]
\end{aligned}
$$

where $\phi_{i j}=\Delta m_{i j}^{2} L /(4 E)$.

A useful and instructive way to present the combined effects of matter and sub-leading terms is in the bi-probability plots of $P\left(\nu_{\mu} \rightarrow \nu_{e}\right)$ versus $P\left(\bar{\nu}_{\mu} \rightarrow \bar{\nu}_{e}\right)$, invented by Minakata and Nunokawa [7]. Fig. 1.2 shows an example of such a plot for a NuMI case. At the larger values of $\sin ^{2} 2 \theta_{13}$ the ellipses associated with the two possible mass hierarchies separate in matter, whereas they are approximately degenerate in vacuum. There is also a significant sensitivity to the CP violating phase, $\delta$. It is the sensitivity to the sign of $\Delta m_{32}^{2}$ and the $\mathrm{CP}$ violating phase in these plots which allows for the determination of these parameters in a sufficiently accurate experiment. For a single experiment there can be a degeneracy in the determined parameters but this degeneracy can be broken by further experimentation.

A high precision measurement of $\nu_{\mu} \rightarrow \nu_{\mu}$ at the atmospheric $\Delta m^{2}$ can also be used to determine the difference from maximal mixing in $\theta_{23}$. This difference is a measure of the breaking of a $\nu_{\mu} \leftrightarrow \nu_{\tau}$ symmetry at some high energy scale. Since matter effects are suppressed in the channel $\nu_{\mu} \rightarrow \nu_{\mu}$ compared to $\nu_{\mu} \rightarrow \nu_{e}$, a comparison of $\nu_{\mu} \rightarrow \nu_{\mu}$ to $\bar{\nu}_{\mu} \rightarrow \bar{\nu}_{\mu}$ is a sensitive test of CPT in the neutrino sector.

In summary the important measurements that could be made by the proposed experiment are

- Observation of $\nu_{\mu} \rightarrow \nu_{e}$ at an L/E of in the range of $10^{2}$ to $10^{3} \mathrm{~km} / \mathrm{GeV}$ which would determine the $\nu_{e}$ role in atmospheric neutrino flavor transitions. In the neutrino oscillation scenario this is a measure of $\sin ^{2} 2 \theta_{13}$.

- Matter effects can be used to distinguish the two mass hierarchies and therefore determine the sign of $\Delta m_{32}^{2}$. 

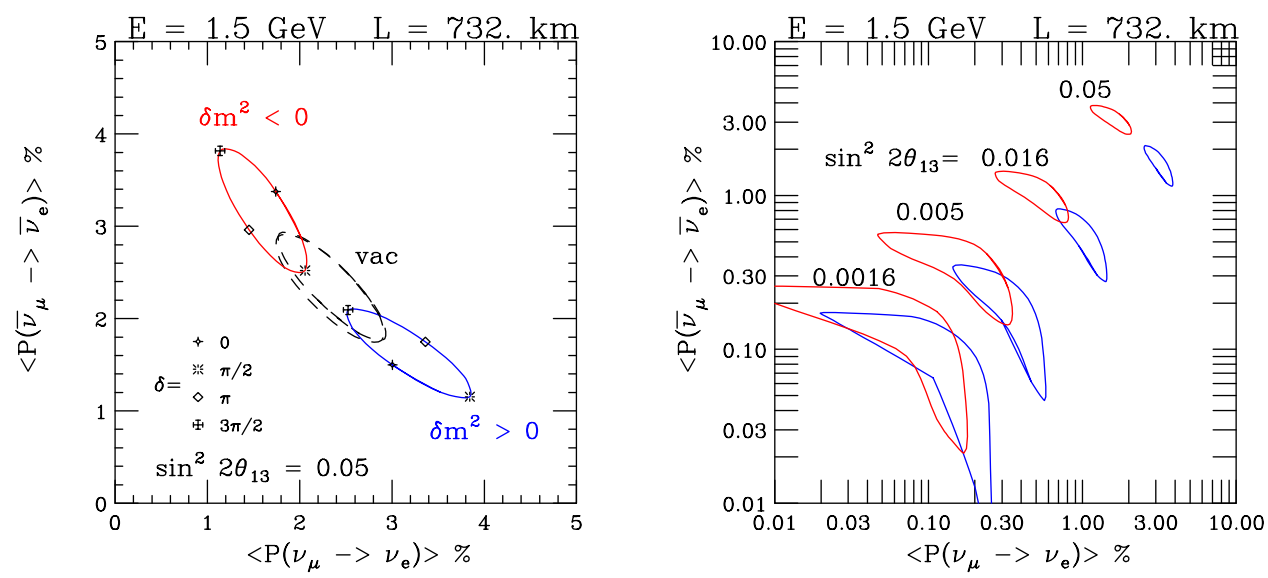

Figure 1.2: The bi-probability plots $P\left(\nu_{\mu} \rightarrow \nu_{e}\right)$ versus $P\left(\bar{\nu}_{\mu} \rightarrow \bar{\nu}_{e}\right)$ assuming a constant matter density of $\rho=3.0 \mathrm{gcm}^{-3}$ for an L/E of $500 \mathrm{~km} / \mathrm{GeV}$. The mixing parameters are fixed to be $\left|\Delta m_{31}^{2}\right|=3 \times 10^{-3} \mathrm{eV}^{2}, \sin ^{2} 2 \theta_{23}=1.0$, $\Delta m_{21}^{2}=+5 \times 10^{-5} \mathrm{eV}^{2}, \sin ^{2} 2 \theta_{12}=0.8$ with the labeled values of $\sin ^{2} 2 \theta_{13}$ and $\delta$.

- For the Large Mixing Angle solution to the solar neutrino puzzle there is sensitivity to the CP violating phase in the channel $\nu_{\mu} \rightarrow \nu_{e}$.

- Precision measurements in $\nu_{\mu} \rightarrow \nu_{\mu}$ channel can measure how close $\theta_{23}$ is to $\pi / 4$, that is maximal mixing. A comparison of $\nu_{\mu} \rightarrow \nu_{\mu}$ to $\bar{\nu}_{\mu} \rightarrow \bar{\nu}_{\mu}$ is a sensitive test of CPT violation since matter effects are suppressed in this channel.

Thus, there is a very rich neutrino physics program to be explored in a $\nu_{e}$ appearance experiment using the NuMI beam. Details of experimental and beam possibilities will be explored in subsequent sections. 


\section{Chapter 2}

\section{The Role of the MINOS Experiment}

The zenith angle distribution of atmospheric $\nu_{\mu}$ interactions observed in the SuperKamiokande experiment gives a very strong indication of neutrino oscillations. A $50 \%$ deficit of upcoming neutrino flux suggests that the mixing angle is very large. A fit to the angular distribution yields the information on the corresponding mass difference $\Delta m_{23}^{2}$ in the range $1.4-4.0 \times 10^{-3} \mathrm{eV}^{2}$. This result is in a good agreement with the initial results of the subsequent accelerator-based long baseline oscillation experiment K2K[8]. Further results from SuperK indicate that the dominant oscillation mode is $\nu_{\mu} \rightarrow \nu_{\tau}$, although a significant admixture of the oscillations into a sterile neutrino is not excluded[9].

The Fermilab Main Injector neutrino beam (NuMI)[10] and the long baseline neutrino oscillations experiment MINOS[11] were proposed in early 90 's to study the 'atmospheric neutrino anomaly' indicated by the results of the IMB[12] and Kamiokande[13] water Cherenkov experiments. An intense neutrino beam is produced from decays of pions and kaons produced by $120 \mathrm{GeV}$ protons interacting with a carbon target. Two parabolic horns focus the produced mesons into a nearly parallel secondary beam of particles which decay inside a 675 meter long evacuated decay pipe.

The MINOS experiment consists of two functionally identical detectors; magnetized iron and solid scintillator calorimeters. The near detector is located at Fermilab $250 \mathrm{~m}$ downstream of the end of the decay pipe. The far detector is located in a Soudan mine at a distance of $735 \mathrm{~km}$ from Fermilab.

Charged current $\nu_{\mu}$ interactions will be recognized in the MINOS detectors via the presence of a long penetrating muon track. Muon momenta will be measured from range with an accuracy of $\sim 6 \%$ for stopping tracks and from bending in the toroidal magnetic field with an accuracy of $\sim 12 \%$. The ironscintillator sandwich provides a calorimetric measurement of the energy of the hadronic final state with the resolution $\Delta E / E \approx 60 \% / \sqrt{E}$. The parent neutrino energy is calculated as a sum of the muon and hadronic final state energies. For 
a neutrino energy around $5 \mathrm{GeV}$ the typical resolution is of the order of $\sim 20 \%$.

Charged current $\nu_{\mu}$ interactions observed in the near MINOS detector will provide a basis for the prediction of the neutrino spectrum expected in the far detector. Owing to the finite size of the decay volume the neutrino spectra in these two detectors are not identical, but the difference is small and can be corrected for. The systematic error on the predicted neutrino spectrum in the far detector is expected to be smaller than $4-5 \%$. An additional systematic cross check of the understanding of the neutrino beam will be provided by precise measurement of the pion and kaon production spectra in the experiment E907, which should enable reliable prediction of the neutrino spectrum in the near detector.

The ratio of the number of charged current $\nu_{\mu}$ interactions observed in the far MINOS detector to the number expected from the spectrum observed at the near detector as a function of the neutrino energy will determine the $\nu_{\mu}$ survival probability at a distance of $L=735 \mathrm{~km}$ as shown in Fig. 2.1. The functional shape of the observed depletion, if any, will be a direct test of the oscillation hypothesis as an underlying physics mechanism. The energy range available to MINOS is well suited to distinguish the oscillation hypothesis from other ad-hoc models put forward to describe the SuperK atmospheric results.

If the observed survival probability will follow the expected $\sin ^{2} \Delta m^{2} L / E$ behavior then the position of the minimum will yield the information on the $\Delta m^{2}$, whereas a deviation of the minimum from one will measure the mixing angle $\sin ^{2} 2 \theta_{23}$. Precise values of the parameters will be determined from a fit to the distribution, after unfolding experimental resolution. Fig. 2.2 shows the expected precision of such a determination.

The MINOS detector is designed for a $\nu_{\mu}$ disappearance experiment and the detector design is optimized for the detection, identification and measurement of $\nu_{\mu}$ charged current interactions. At the same time, it offers a considerable potential for a search for $\nu_{\mu} \rightarrow \nu_{e}$ oscillations. The longitudinal sampling of the MINOS calorimeter, $2.5 \mathrm{~cm}$ of iron, represents $1.5 X_{0}$ whereas the transverse granularity of the readout $4 \mathrm{~cm}$, is of the order of the Moliere radius. Electrons produced in $\nu_{e}$ charged current interactions produce a characteristic energy deposition with small spatial extent and high energy density. This pattern is quite different from the pattern produced by charged hadrons, but indistinguishable from the energy deposition of a neutral pion.

Several studies have demonstrated that MINOS detector can identify $\nu_{e}$ charged current interactions[14] with efficiency of the order of $25 \%$ while providing a rejection against neutral current background by a factor of 65 . This will allow the MINOS experiment to detect $\nu_{\mu} \rightarrow \nu_{e}$ oscillations, if they occur at the level of few per cent, or set a limit on $\left|U_{e 3}\right|^{2}$ of the order of 0.01 . This would represent a factor of 3 improvement over the current limit from the $\mathrm{CHOOZ}$ experiment for the central value of $\Delta m_{\text {atmos }}^{2}$ and even bigger improvement for lower values of $\Delta m_{\text {atmos }}^{2}$ still allowed by SuperK and other experiments. Table 2.1 shows a composition of the expected background sample in the MINOS detector for an exposure corresponding to $8 \times 10^{20} \mathrm{POT}$ in the low energy beam. 


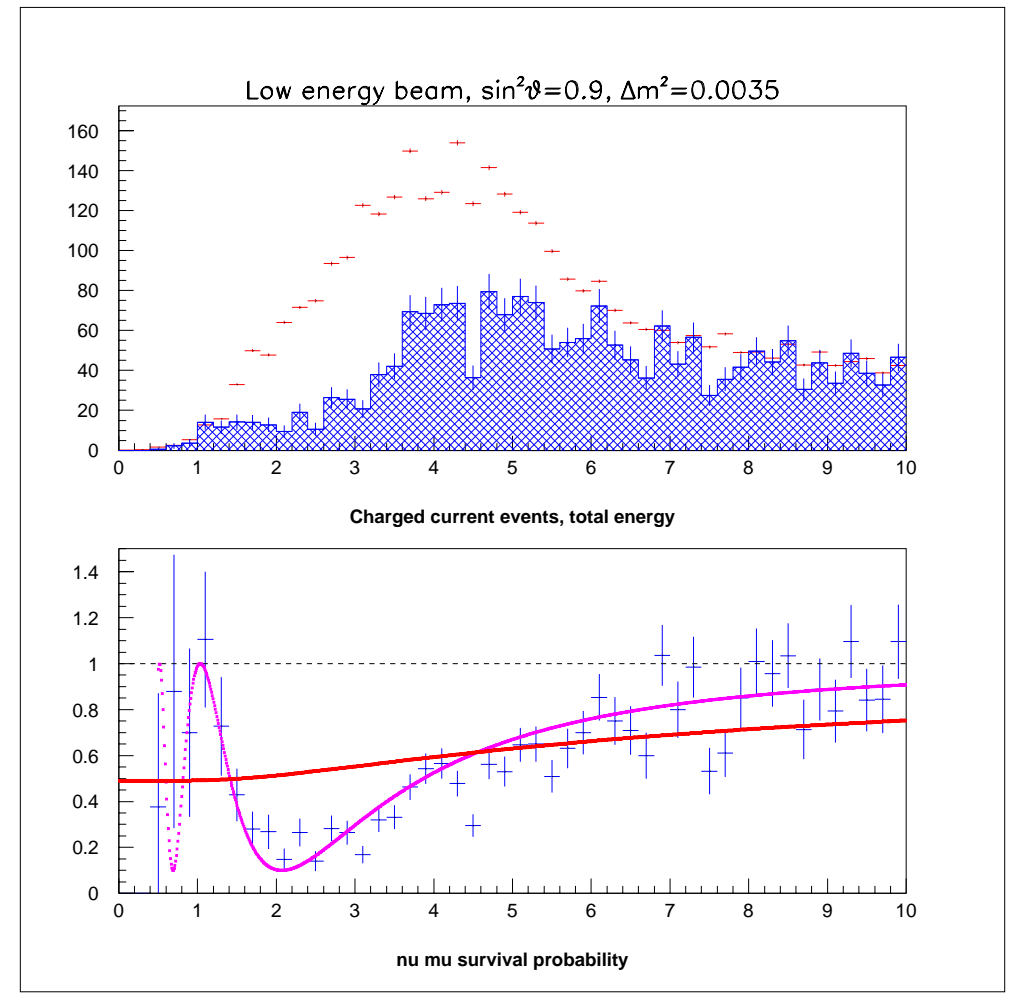

Figure 2.1: Top: expected spectrum of $\nu_{\mu}$ charged current events in the far MINOS detector for $8 \times 10^{20}$ protons and the observed spectrum in case of oscillations with $\Delta m_{32}^{2}=0.0035 \mathrm{eV}^{2}$ and $\sin ^{2} 2 \theta_{23}=0.9$, shaded histogram. Bottom: ratio of the observed and the expected energy distributions, i.e. $\nu_{\mu}$ survival probability, as a function of the neutrino energy. Magenta curve is the shape expected for the neutrino oscillations, red curve is the expectation of the neutrino decay model consistent with the SuperK data.

Relatively high background levels, exceeding the unavoidable intrinsic $\nu_{e}$ component of the beam are partly related to the relatively poor granularity of the MINOS detector. A significant factor is due to the mismatch between the neutrino spectrum of the NuMI beam and the oscillation probability: a significant fraction of neutrinos present in the beam do not contribute to the signal, whereas it produces background events. Improvement of the $\nu_{\mu} \rightarrow \nu_{e}$ oscillations limit with the exposure time will be very slow and it will be limited by the systematic uncertainties in the background estimate.

To attain higher sensitivity to the $\nu_{\mu} \rightarrow \nu_{e}$ oscillations the following steps, 


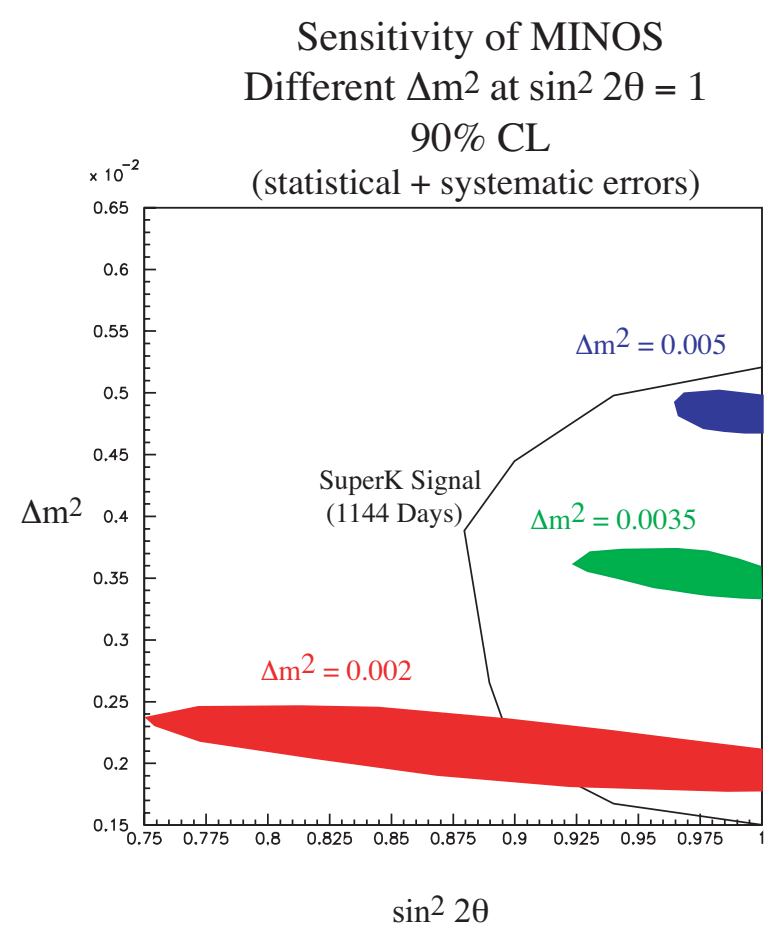

MINOS Approved (4.0E20/yr, 2 years, Low-Energy)

Feldman/Cousins Frequentist $\quad$ (R. Bernstein)

Figure 2.2: Expected precision of the determination of the oscillations parameters, $\Delta m_{32}^{2}$ and $\sin ^{2} 2 \theta_{23}$ with low energy beam for different values of $\Delta m_{32}^{2}$. Horizontal axis is $\sin ^{2} 2 \theta_{23}$, vertical axis is $\Delta m_{32}^{2}$ in $\mathrm{eV}^{2}$.

with respect to the MINOS experiment, are desirable:

- reduce the $\mathrm{NC}$ background contribution by a better match of the neutrino beam spectrum to the oscillation probability curve

- reduce/eliminate the background due to $\nu_{\mu} \rightarrow \nu_{\tau}$ oscillations by reducing the neutrino flux above the $\tau$ production threshold

- improve efficiency for the signal detection and reduce the background from $\mathrm{NC}$ interaction by an optimization of the detector for the $\nu_{e}$ detection and identification

- improve statistics by an increase of the numbers of protons on the neutrino target and/or an increase of the detector size (preferably both) 


\begin{tabular}{|c|c|c|c|c|c|}
\hline signal & beam $\nu_{e}$ & $\nu_{\mu} \mathrm{CC}$ & $\nu_{\mu} \rightarrow \nu_{\tau}$ & $\mathrm{NC}<10 \mathrm{GeV}$ & $\mathrm{NC}>10 \mathrm{GeV}$ \\
\hline 8.5 & 5.6 & 3.9 & 3.0 & 15.7 & 11.5 \\
\hline
\end{tabular}

Table 2.1: Number of the expected signal $\nu_{e}$ events, with $\left|U_{e 3}\right|^{2}=0.01$, and contributions to the background samples for $8 \times 10^{20}$ POT exposure of the MINOS detector in the low energy beam. 


\section{Chapter 3}

\section{Possible Evolution of Neutrino Physics}

While the preceding chapters gave the motivation for the proposed neutrino oscillation experiment solely from the physics point of view, it may be useful to put this endeavor into the broader context of the research programme of the USA, and even of the world-wide programme.

In the USA, a strong tradition of successful experimentation with accelerator neutrinos exists at Fermilab, which has culminated with the high-precision results from CCFRR and $\mathrm{NuTeV}$ on the nucleon structure functions and on the electroweak mixing angle. With the enthusiasm for the prospects offered by the newly discovered neutrino oscillations, MINOS and MiniBooNE will take over and guarantee interesting research and important results in the next five to ten years. Yet, the development in neutrino physics has proven fast, many new initiatives are being discussed also in Europe and Japan. It is important for the USA, and in particular for Fermilab, to plan the future in accelerator neutrino physics beyond MINOS and MiniBooNE now.

With a view to complying with scarce resources the concurrent use of the NuMI beam for a second 'off-axis' detector appears as optimal choice, combining an effective use of existing facilities with both a significant and far-reaching extension of the physics agenda and a relatively modest expenditure. While this program is expected to face some healthy competition from overseas activities, it is fair to say that by and large it fits very well into a largely complementary world-wide effort to advance our understanding of the physics of neutrino oscillations. 
The first outstanding issue is pinning down the oscillation parameters of solar neutrinos. The recent spectacular results from SNO[15] (located in the Sudbury mine, Canada) have finally confirmed that the solar neutrino deficit is not of astrophysical origin, but due to the oscillation of electron-neutrinos into other active neutrino flavors. Remaining ambiguities between different domains of oscillation parameters are expected to be resolved within a few months by the KamLAND[16] reactor neutrino experiment (located in the Kamioka mine, Japan), and subsequently confirmed by the BOREXINO[17] experiment (located in the Gran Sasso Laboratory, Italy). From then on, the challenge will be to measure the relevant mixing parameters $\Delta m_{12}^{2}$ and $\sin ^{2} 2 \theta_{12}$ as precisely as possible, a program to which accelerator neutrinos are not expected to contribute in the foreseeable future.

The main drive to measure the oscillation parameters of atmospheric neutrinos, $\Delta m_{23}^{2}$ and $\sin ^{2} 2 \theta_{23}$, is expected to come from MINOS. MiniBooNE is expected to rule out the evidence from LSND, or else a new and equally complicated and interesting situation will have to be faced, which is not anticipated here. The second outstanding issue will be to confirm a non-zero value of the third mixing parameter in a scheme with three and only three active neutrino flavors, $\sin ^{2} 2 \theta_{13}$, or else reduce its current upper bound of $\sim 0.1$ to as low a value as possible. In this area, parallel to the ideas put forward in this LoI, several initiatives are currently under discussion also in Europe and Japan, albeit with grossly varying chances of realization.

Table 3 attempts to summarize these initiatives and compare their physics objectives. The table also gives order-of-magnitude prices and an estimate of the earliest time of realization. It should be understood that this assessment constitutes at best an educated guess and can in no way be taken as an commitment of the proponents themselves. The entry 'F2S' of the table refers to the experiment proposed in this letter. The other entries in the table are now discussed in turn:

- An Expression of Interest was recently put forth to look for $\nu_{\mu} \rightarrow \nu_{e}$ oscillations using the CERN neutrino beam aimed at Gran Sasso Laboratory[19], currently under construction, with an off-axis Cherenkov detector located deep underwater in the Gulf of Taranto, $1200 \mathrm{~km}$ from CERN[18]. The offaxis angle of about $2 \mathrm{deg}$ is such that the corresponding neutrino energy of $0.8 \mathrm{GeV}$ would place the second oscillation maximum at this location. The detector would be movable and would take data at several baselines. This initiative, is listed in the tables as 'C2GT'. The CNGS beam would have to be tuned to a much lower energy than currently being planned for the Gran Sasso program. This would imply either a change in the current plans or a delay till 2011 or later when the OPERA experiment is scheduled to be finished. 
- In Japan, a $50 \mathrm{GeV}$ proton accelerator with very high intensity, the Japanese Hadron Facility (JHF)[20], has been approved and is under construction. This facility is due to be completed around 2007 and has a first-stage design beam power of $0.77 \mathrm{MW}$. There are now rather detailed plans to construct a neutrino beam[20], most likely an off-axis beam at 2 deg with an energy centered around $0.7 \mathrm{GeV}$, aimed at the Super-K detector $295 \mathrm{~km}$ away. Even though the neutrino program at the JHF is not officially approved as yet it is anticipated that the formal approval will be obtained within a year. This proposed experiment is listed as 'JHF2K' in the table. A more detailed comparison of this program with the proposed NuMI program is given elsewhere in this document. The JHF program envisions also a second phase some time later. The accelerator itself would have its intensity upgraded to $4.0 \mathrm{MW}$. In addition, plans are being formulated to construct a new water Cherenkov detector in the megaton range, Hyper-K, near the Super-K detector so that the same neutrino beam could be exploited by both detectors. The program based on such a detector with a higher intensity accelerator should be able to address the issues of $\mathrm{CP}$ violation if $\theta_{13}$ is not too low. This second phase of the Japanese program is termed 'JHF2K II' in the table.

- There has been a discussion at CERN about building a superconducting proton linac re-using the LEP RF cavities. It would deliver a very high flux of protons of $2.2 \mathrm{GeV}$ kinetic energy[21]. Such an accelerator could produce an intense wide-band neutrino beam with an average neutrino energy of $300 \mathrm{MeV}$. In parallel, the idea was put forth to construct a large water Cherenkov detector of Super-K type in the Fréjus tunnel, utilizing the opportunity of doing that relatively cheaply while other construction was proceeding in that tunnel. The energy of the proposed beam and the CERN Fréjus distance would be a good match to search for the $\nu_{\mu} \rightarrow \nu_{e}$ oscillations at the first maximum of oscillations. Both of these efforts are still in the design and discussion stage, they require a considerable financial expenditure and face therefore major hurdles arising from the very tight financial situation at CERN which is imposed by the priority which is given to the LHC program. They are termed ' $\mathrm{C} 2 \mathrm{~F}$ ' in the table.

- The novel idea of a 'Beta-Beam' was recently put forth to create pure $\nu_{e}$ and $\bar{\nu}_{e}$ beams from decays of radioactive nuclei, ${ }^{18} \mathrm{Ne}$ and ${ }^{6} \mathrm{He}[22]$. The nuclei would have to be accelerated to a $\gamma$ around 75 and would give wellcollimated wide-band neutrinos in the $300 \mathrm{MeV}$ range. The plan would be to aim this beam at the water Cherenkov detector in the Fréjus tunnel, discussed above. This initiative is indicated as ' $\mathrm{C} 2 \mathrm{~F}+\mathrm{BB}$ ' in the table.

Both a new detector and a new storage ring-accelerator with long straight sections would have to be constructed to make such a program a reality. While interesting in its own right, the realization of such a project appears unrealistic because of the very high expenditure associated with it.

- The ultimate challenge in the physics of neutrino mixing is to determine 
the existence and size of leptonic CP-violation. It is universally agreed that the "neutrino factory[23] based on novel muon storage rings with long straight sections gives the best access to this potentially very important phenomenon. It goes almost without saying that any other aspect of the oscillation $\nu_{\mu} \rightarrow \nu_{e}$ which would be left open from earlier experiments, would be easily and decisively addressed by the neutrino factory.

Over the last several years there have been intensive studies of the physics potential of a high energy muon storage ring with energy up to $50 \mathrm{GeV}$ with long straight sections.

The beam of the neutrino factory, for stored $\mu^{+}$would consist of $\nu_{e}$ 's and $\bar{\nu}_{\mu}$ 's with a well-defined energy spectrum. The $\nu_{\mu} \rightarrow \nu_{e}$ oscillations would give a clean and experimentally well accessible signature of "wrong sign" muons.

The technical studies of such a machine identified a number of difficult challenges associated with obtaining the desired high intensity of the stored muon beams. The projected cost appears to be in the range of two billion dollars. Therefore, it is unlikely that there will be a significant movement towards construction of a neutrino factory unless and until $\theta_{13}$ is shown to be large enough to allow detailed studies of $\mathrm{CP}$ violation. Neutrino factories are entered as ' $\nu \mathrm{F}$ ' in the table.

The current best limits on $\nu_{\mu} \rightarrow \nu_{e}$ oscillations in the atmospheric $\Delta m^{2}$ range come from reactor experiments, CHOOZ[2] and Palo Verde[24]. They study a related process, i.e. disappearance of $\nu_{e}$ 's by measuring flux and energy spectrum of $\nu_{e}$ 's some distance away from the reactor and comparing it with the predicted one. Statistical and systematic errors contribute roughly equally in both of these experiments; thus improving the sensitivity will require advances on both of those fronts. Searching for a small effect in a disappearance experiment requires very good control of systematics.

Recently there has been a proposal for a new experiment to search for $\nu_{e}$ disappearance with a sensitivity roughly a factor of 8 better than the $\mathrm{CHOOZ}$ limit. This experiment[25], based on the Krasnoyarsk reactor, would utilize two identical detectors, 50 tons each, at two different locations. The detection method would be similar to the one used by the KamLAND and BOREXINO experiments.

It is not clear whether this proposal will be realized. The proponents plan on performing first a pilot experiment, with smaller detectors at shorter distances, to investigate the LSND effect. There is also a possibility that the Krasnoyarsk reactor will be decommissioned sometimes in the near future. 


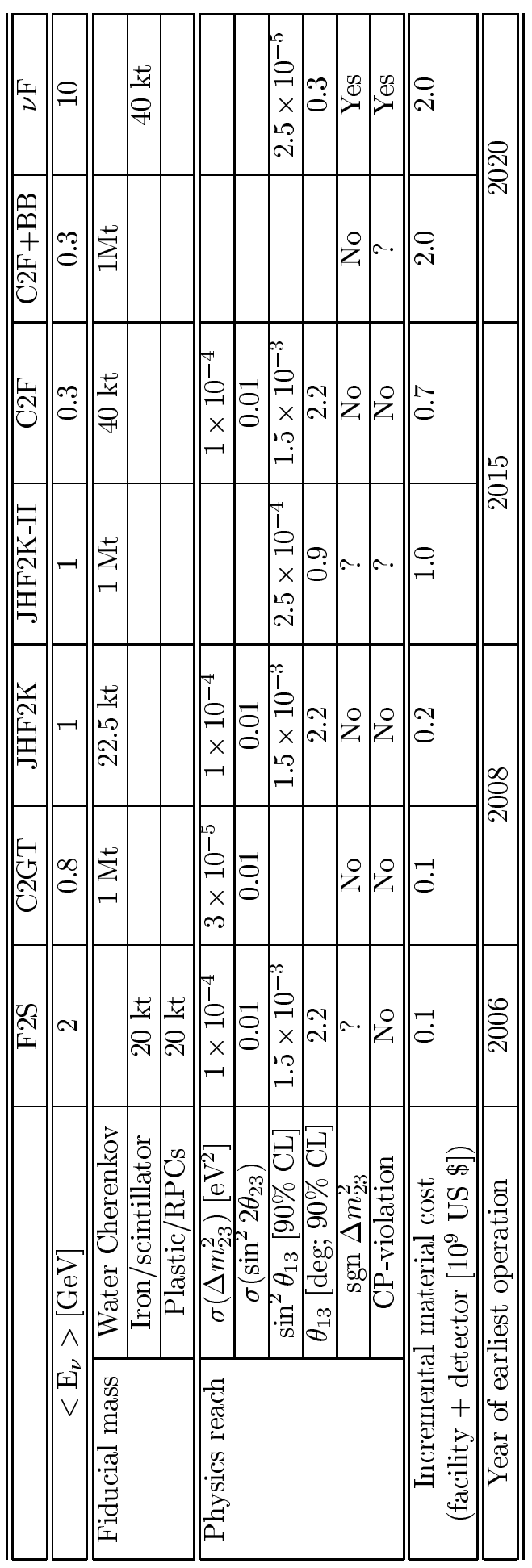




\section{Chapter 4}

\section{Off-Axis NuMI Neutrino Beam}

Construction of the NuMI neutrino beam is currently underway at Fermilab. This beam directs $120 \mathrm{GeV}$ protons from the Main Injector towards a $96 \mathrm{~cm}$ long graphite target. The secondary pions and kaons produced on this target are captured and focused by two parabolic magnetic horns pulsed at $200 \mathrm{kA}$. They decay in an evacuated region $675 \mathrm{~m}$ in length and $2 \mathrm{~m}$ in diameter to yield a very pure beam of muon neutrinos.

The unique feature of the NuMI neutrino beam is its flexibility. The neutrino energy spectrum can be selected as 'low', 'medium' or 'high energy' by adjusting the relative positions of the focusing horns and the target[10]. The neutrino beam energy can be also changed in a continuous fashion by moving the target with respect to the first horn[26]. The expected neutrino spectra at the MINOS far detector site are shown in Figure 4.1 for the NuMI low, medium, and high energy beam tunes.

The NuMI beam line is designed for a proton intensity of $4 \times 10^{13}$ protons per pulse every $1.9 \mathrm{sec}$. - roughly $0.4 \mathrm{MW}$. At the design intensity, NuMI should collect $3.7 \times 10^{20}$ protons per year, which will produce 470 neutrino interactions, in the absence of oscillations, per year per kt of detector mass at the far MINOS detector location in the low-energy beam configuration.

Excavation of the NuMI beam line is complete. Commissioning of the beam is expected to begin in the first months of 2005 . 

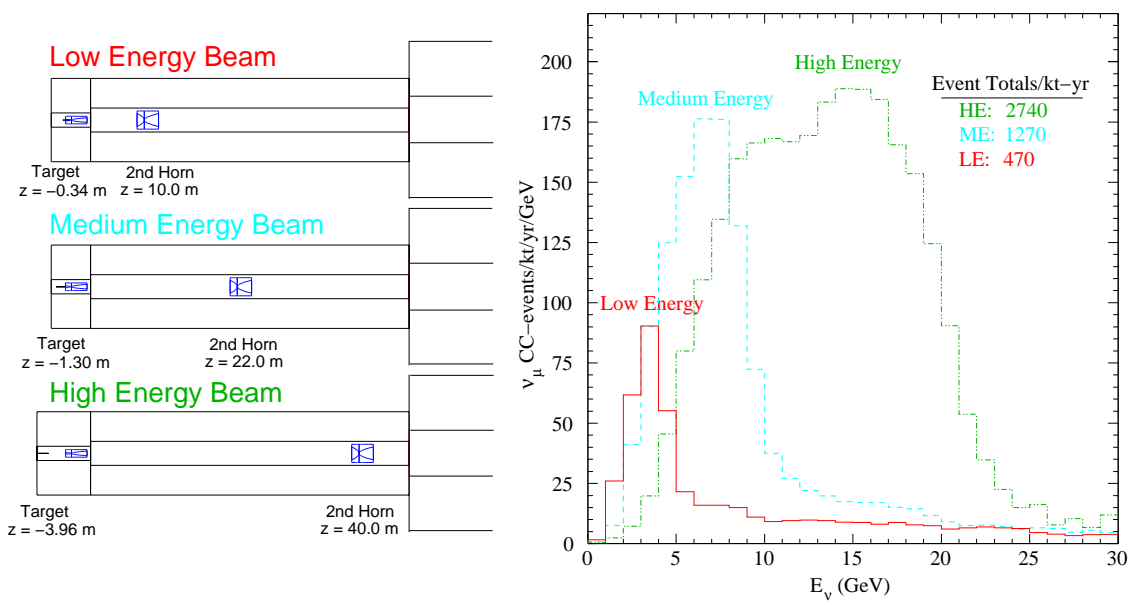

Figure 4.1: Left: The locations of the target and second horn for the NuMI low, medium, and high energy wide-band configurations. Right: Expected neutrino spectra at the MINOS far detector site in each of the NuMI beam tunes.
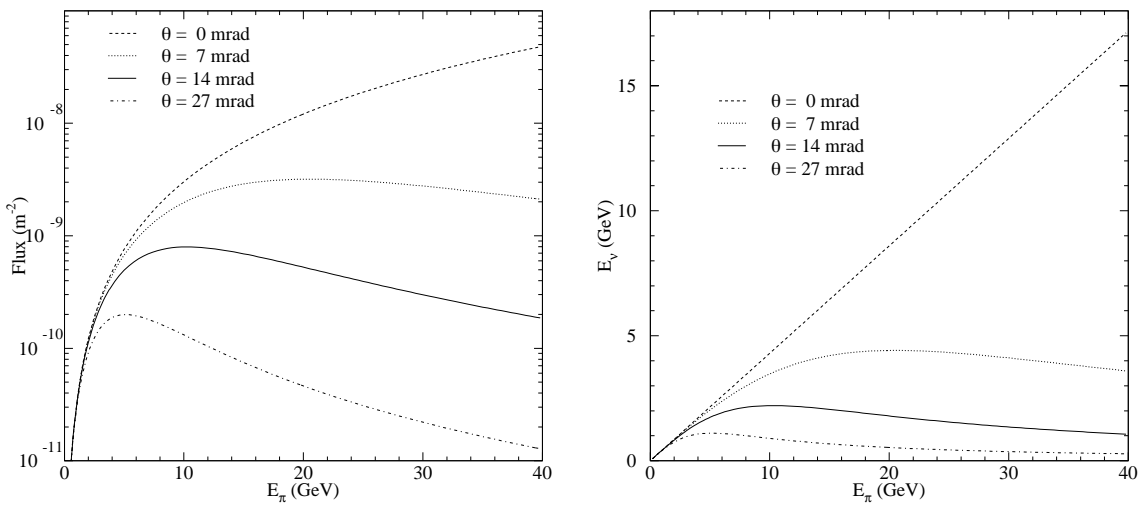

Figure 4.2: Left: The neutrino flux from a pion of energy $E_{\pi}$ as viewed from a site located at an angle $\theta$ from the beam axis. The flux has been normalized to a distance of $z=735 \mathrm{~km}$. Right: The energy of the neutrino produced at angle $\theta$ relative to the pion beam direction as a function of the pion energy. 

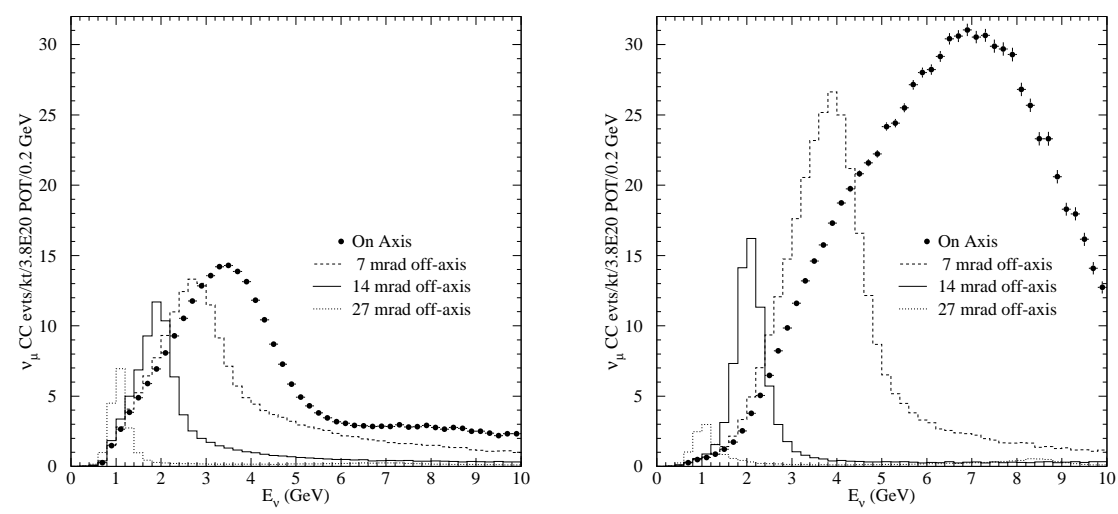

Figure 4.3: CC muon neutrino events rates expected under 'no oscillation' hypothesis at a distance of $735 \mathrm{~km}$ from Fermilab and at various transverse locations for the NuMI low-energy beam configuration (left) and medium-energy beam configuration(right).

\subsection{NuMI Off-Axis Neutrino Fluxes}

Due to the isotropic decays of the focused pions and kaons, the neutrino beam produced at NuMI will have broad angular distribution. For small angles, the flux and energy of a neutrino produced from the two body decay of a pion in flight are given in the lab frame by:

$$
\mathcal{F}=\left(\frac{2 \gamma}{1+\gamma^{2} \theta^{2}}\right)^{2} \frac{A}{4 \pi z^{2}}, E_{\nu}=\frac{0.43 E_{\pi}}{1+\gamma^{2} \theta^{2}},
$$

where $\theta$ is the angle between the pion direction and the neutrino direction, $E_{\pi}$ is the energy of the parent pion, and $m_{\pi}$ is the mass of the pion and $\gamma=E_{\pi} / m_{\pi}$. $A$ and $z$ are the detector cross-sectional area and distance from decay point. As can be seen in the Figure 4.2, the neutrino flux peaks in the forward direction for all values of $E_{\pi}$; this is why the MINOS detectors are placed on axis. As the angle to the beam direction increases, however, the relationship between the pion energy and neutrino energy flattens, with all pions yielding neutrinos of roughly the same energy.

It is possible to construct a nearly mono-energetic neutrino beam by viewing the NuMI beam at a location off the beam axis. This is an implementation of a concept developed for the proposed experiment E-889 at Brookhaven[27]. Because all pions contribute neutrinos of roughly the same energy, it is possible to compensate the reduction in the flux factor. Figure 4.3 shows the neutrino 

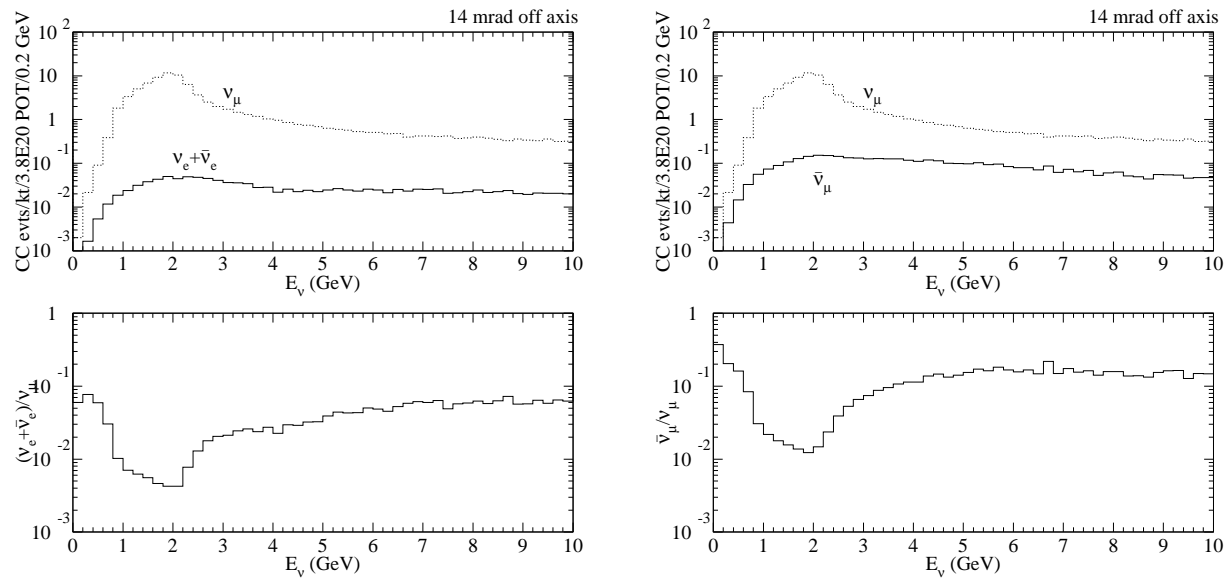

Figure 4.4: The expected energy distribution of the electron neutrino (left) and wrong-sign muon neutrino (right) components of the NuMI off-axis beams, low energy beam.

spectra of the NuMI neutrino beam at locations 5,10 , and $20 \mathrm{~km}$ (corresponding to the decay angles of 7,14 , and $21 \mathrm{mrad}$ ) off the NuMI beam axis at a distance of $735 \mathrm{~km}$ from the target[28]. As can be seen in Fig. 4.3, it is possible to increase the neutrino yield in the 1-2 $\mathrm{GeV}$ region by placing the detector off the beam axis, while at the same time reducing the high-energy component of the neutrino beam.

The off-axis beam intensity is higher, the beam energy spread is smaller and the high energy tail is smaller for the medium energy beam tune than for the low energy one. The intensity of the off-axis beam produced in the high energy beam configuration is reduced significantly in comparison with that of the medium energy setting due to the kinematical reduction of the flux.

The low energy peak of the off-axis beam spectrum is produced almost exclusively by decays of pions; hence the prediction of the neutrino flux is quite insensitive to the experimental uncertainties of the ratio of $K / \pi$ production cross sections. At the typical distance of $10 \mathrm{~km}$ from the nominal beam axis the resulting spectrum of the neutrino beam is much narrower than the expected maximum of the oscillation probability. The total number of events in the peak region can be predicted on the basis of the near MINOS detector with an accuracy better than $2 \%[29]$. 

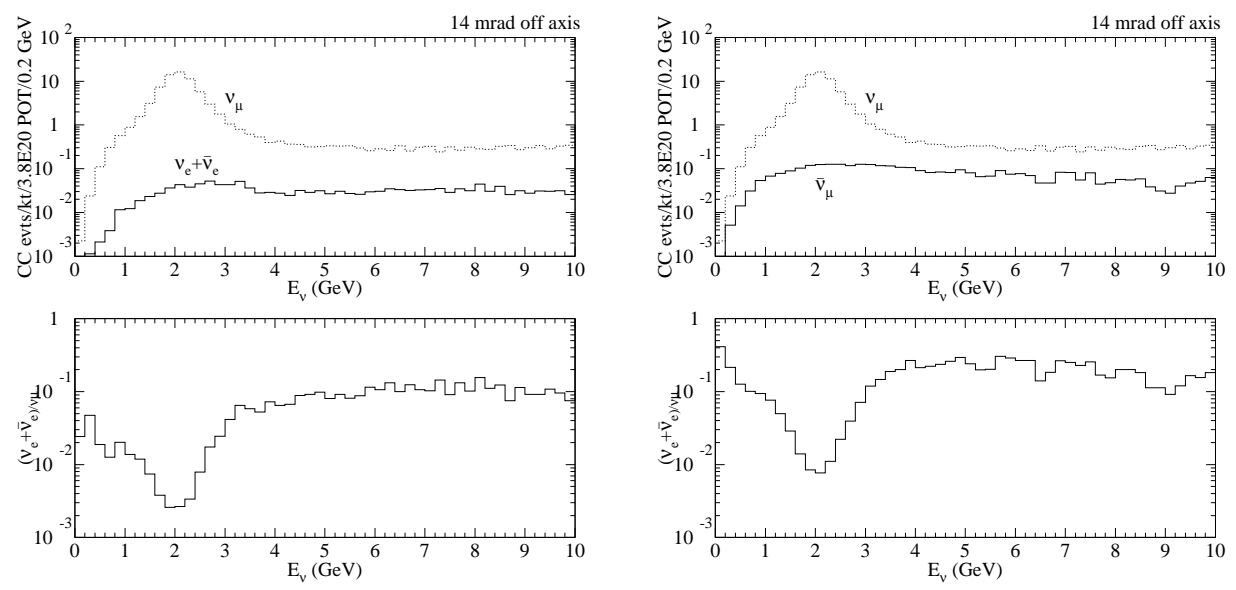

Figure 4.5: The expected energy distribution of the electron neutrino (left) and wrong-sign muon neutrino (right) components of the NuMI off-axis beams, medium energy beam.

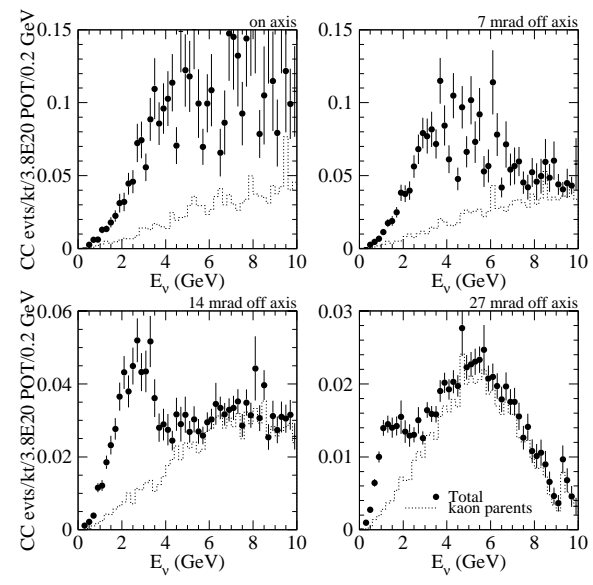

Figure 4.6: Spectra of the electron neutrino component of the beam at different off-axis angles. Points represent the total background and a histogram shows the component due to $K_{e 3}$ decays. 


\subsection{Backgrounds}

The neutrino event rates and energy distribution depend on the detector location,its distance from Fermilab, and the distance from the nominal beam axis, but their qualitative features are very similar. As an example, we show the expected composition of the off-axis NuMI neutrino fluxes in Figure 4.4 and 4.5 at a distance of $L=735 \mathrm{~km}$. The $\bar{\nu}_{\mu}$ component of the beam is due to the decays of high energy $\pi^{-}$traversing the opening of the magnetic horn and not being de-focused by the horn system. This 'wrong' sign beam component contributes roughly $1 \%$ of events at the peak of the neutrino flux. More importantly, the component of electron neutrinos is kept to roughly $0.5 \%$ in the peak of the neutrino flux. The $\nu_{e}$ component of the beam is due to $K_{e 3}$ decays and to the decays of tertiary muons. The $\nu_{e}$ 's from the $\mathrm{K}$ decays have much higher energies than the main peak of the $\nu_{\mu}$ 's, whereas the neutrinos from the $\mu$ decays populate the low energy part of the spectrum. Fig. 4.6 shows that $\sim 80 \%$ of the $\nu_{e}$ background at the peak of the $\nu_{\mu}$ flux distribution is due to muon decays. This component of the background should be well constrained by measurements of the muon fluxes and muon neutrino spectra at the Fermilab site. The systematic error of the extrapolation to the off-axis detector is of the order of $5-6 \%$.

It is worth noting that that the relative contribution of backgrounds is somewhat smaller for the medium energy beam setting than for the low energy one.

\subsection{Antineutrino Beams}

An antineutrino beam, in addition the neutrino beam, is an important tool for the investigation of the neutrino mass hierarchy and in the search for $\mathrm{CP}$ violation in the neutrino sector.

The direction of the current through the NuMI focusing horns can be reversed to produce an anti-neutrino beam rather than a neutrino beam. The expected anti-neutrino event rates are shown in Figures 4.7 and 4.8. The events rates in anti-neutrino mode are $\sim 30 \%$ of those expected when running in neutrino mode. Most of the rate reduction is due to the lower anti-neutrino cross-sections, as shown in Fig. 4.11, and the rest is related to the lower production of $\pi^{-}$relative to $\pi^{+}$in proton-nucleus interactions.

The compositions of the anti-neutrino beams are shown in Figures 4.9 and 4.10. Note that while the wrong-sign muon contamination is worse in anti-neutrino mode, the electron neutrino rates are still roughly $0.3-0.5 \%$ at the peak of the 

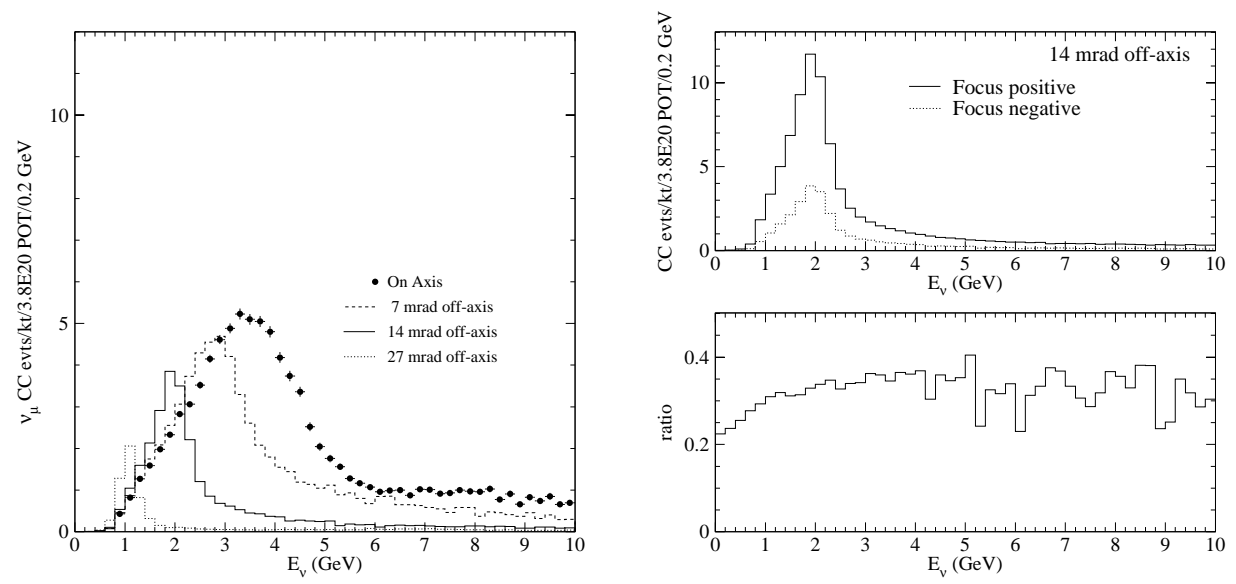

Figure 4.7: Left: The muon anti-neutrino event rates expected at the distance $L=735 \mathrm{~km}$ from Fermilab in the NuMI low energy beam with reversed horn currents at various transverse locations. Right: Comparison of the anti-neutrino event rates to those obtained when running in anti-neutrino mode.
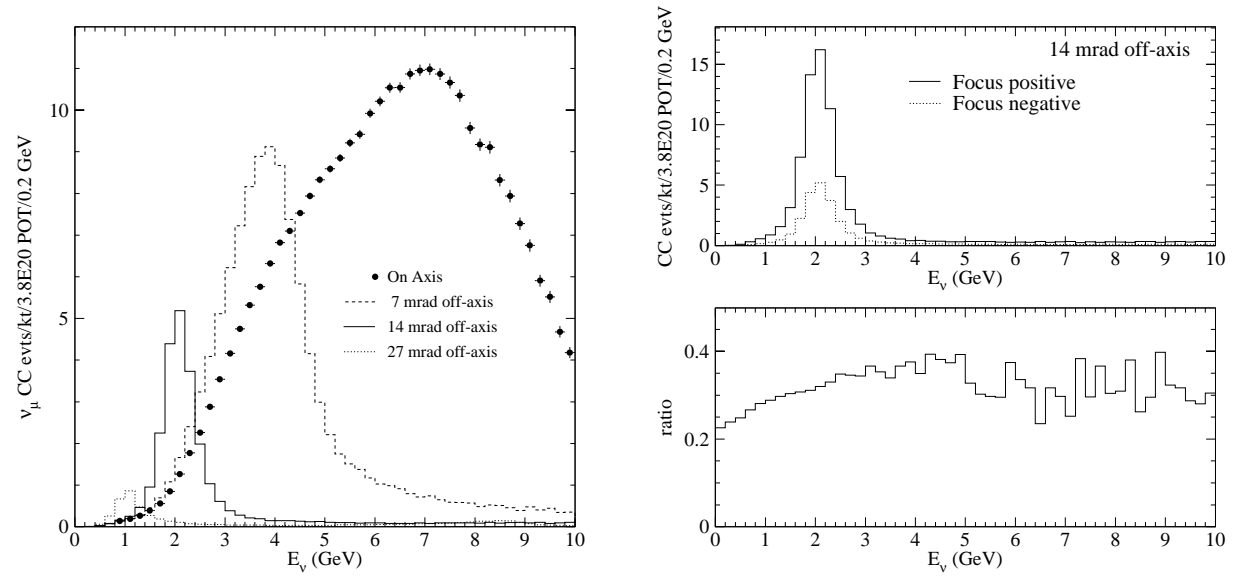

Figure 4.8: Left: The muon anti-neutrino event rates expected at the distance $L=735 \mathrm{~km}$ from Fermilab in the NuMI medium energy beam with reversed horn currents at various transverse locations. Right: Comparison of the antineutrino event rates to those obtained when running in anti-neutrino mode. 

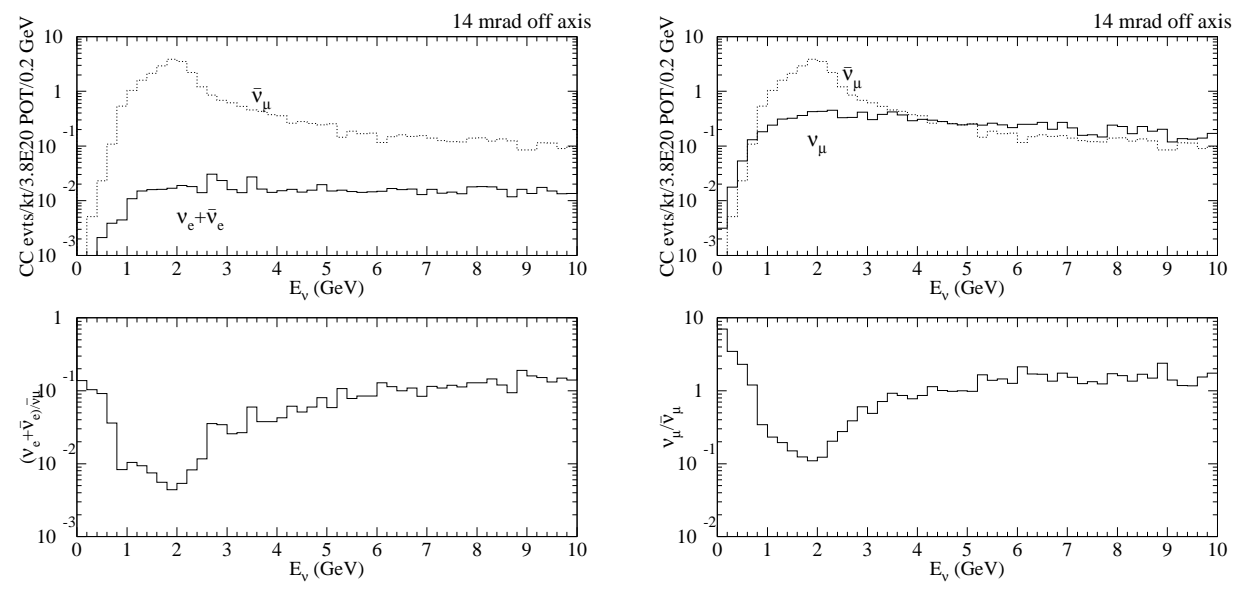

Figure 4.9: Composition of the off-axis antineutrino beam, low energy beam configuration. Left shows the electron neutrino component. Right shows the wrong-sign muon component.
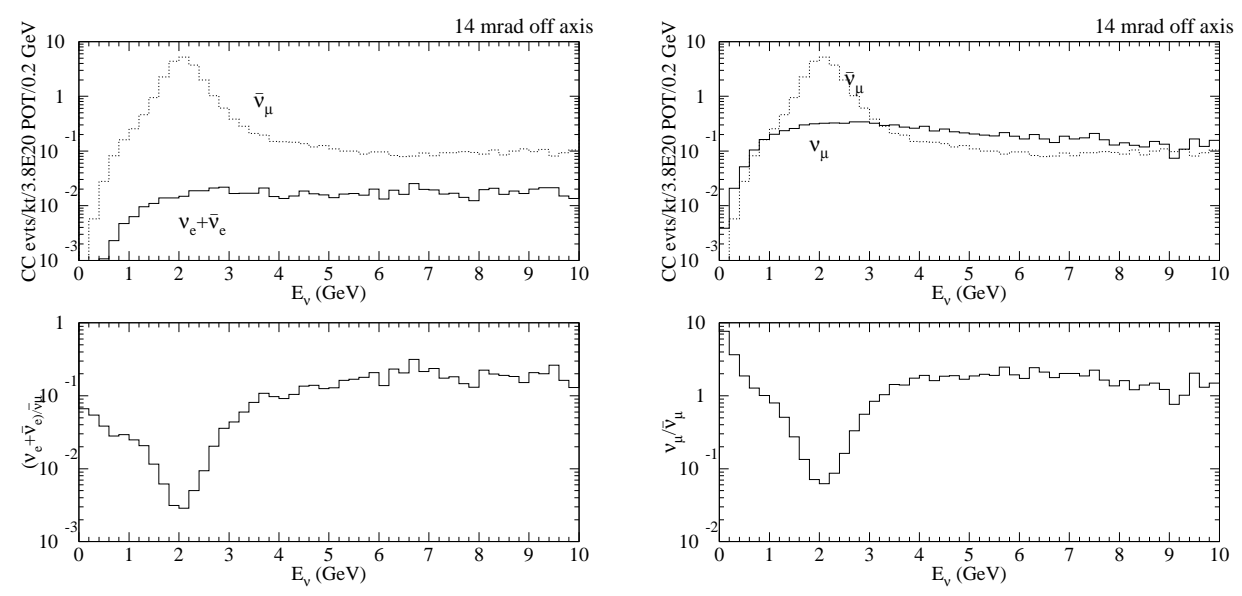

Figure 4.10: Composition of the off-axis antineutrino beam, medium energy beam configuration. Left shows the electron neutrino component. Right shows the wrong-sign muon component. 


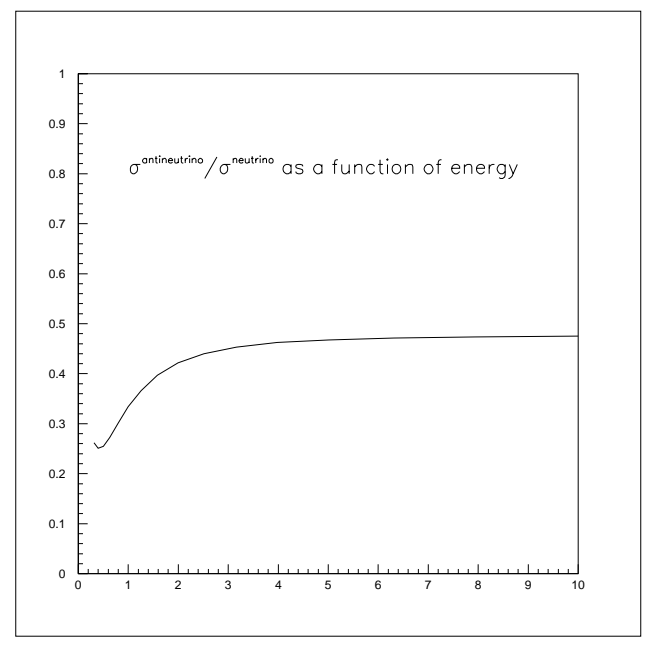

Figure 4.11: Ratio of antineutrino and neutrino CC cross sections as a function the the neutrino energy

neutrino spectrum.

\subsection{Proton Economics}

In its initial phase of operation NuMI will have to accomodate the $\bar{p}$ production cycle. Six consecutive batches of $8 \mathrm{GeV}$ protons from the Booster will be injected into the Main Injector to be accelerated to $120 \mathrm{GeV}$, with a cycle time of $1.9 \mathrm{~s}$. One batch will be extracted for $\bar{p}$ production, and the remaining five will be extracted onto the NuMI target in about $8 \mu s$.

At present, with the Booster running routinely at $4.5 \times 10^{12}$ protons/batch, the maximum intensity one can expect is limited to $2.3 \times 10^{13}$ protons/cycle. This would provide about $2 \times 10^{20}$ protons/year, assuming 5000 hours/year of operation.

There is no obvious path to increase the total proton flux, but several ideas are being actively investigated. Both the feasibility of Booster upgrades to increase the number of protons/batch and the possible gain provided by proton stacking techniques in the Main Injector (slip-stacking and RF barrier stacking), to increase the number of protons per Main injector cycle, are being currently evaluated. Another path would be an upgrade of the power supplies and the RF system of the Main Injector to allow shorter operation cycles down to $1 \mathrm{~s}$. 
A proton intensity working group has been jointly appointed by the Fermilab Directorate and the MINOS experiment to produce a roadmap to help maximize the integrated number of protons to be delivered onto the NuMI target. 


\section{Chapter 5}

\section{$\nu_{\mu} \rightarrow \nu_{e}$ Oscillation Appearance Experiment}

\subsection{Statement of the challenge}

The challenge for future experiments is to observe $\nu_{\mu} \rightarrow \nu_{e}$ oscillations in the atmospheric neutrino $\Delta m^{2}$ range down to the level of a few parts per mil. The CHOOZ experiment gives a limit on $\nu_{e}$ disappearance probability of about 0.1 . This translates into a limit on the $\nu_{e}$ appearance probability of 0.05 . MINOS is expected to improve this by a factor of $2-3$, setting the aforementioned scale of few parts per mil. There are no clear theoretical guidelines as to the most likely value of this parameter.

Charged current $\nu_{e}$ interactions can be identified by the presence of an electron in the final state. The experimental backgrounds to the $\nu_{\mu} \rightarrow \nu_{e}$ oscillation signals arise from two general sources. There are genuine events with electrons resulting from the intrinsic $\nu_{e}$ component in the beam and from $\tau$ decays in the charged current $\nu_{\tau}$ interactions from $\nu_{\mu} \rightarrow \nu_{\tau}$ oscillations. In addition there are potentially mis-identified $\mathrm{NC}$ events or high y $\nu_{\mu} \mathrm{CC}$ events where one or more $\pi^{0}$ 's in the final state masquerade as an electron.

The intrinsic $\nu_{e}$ 's in the beam come from $\mu$ decays and $K_{e 3}$ decays (both charged and neutral). They are of the order of $0.5-1.0 \%$ of $\nu_{\mu}$ 's, but can be reduced further by an appropriate energy cut. The $\tau$ decay contamination has a strong energy dependence; the easiest way to reduce it is to have a significant fraction of the $\nu_{\mu}$ flux below the $\tau$ production threshold. $K_{e 3}$ contamination is typically 
of the order of $1 / 5$ of the $\mu$ decay contamination in the experiments being considered, as shown in Fig. 4.6.

The experimental challenge can be separated into two parts:

- reducing these two backgrounds as much as possible

- measuring them as accurately as possible so that the principal ultimate uncertainty comes from the statistical fluctuations in the event sample of interest

The background from $\nu_{e}$ 's can only be reduced by good energy resolution since the $\nu_{e}$ 's from background sources have a broader energy spectrum than the potential signal. The NC background, however, can be reduced by a well designed detector. The challenge is to suppress it to a level comparable or lower than the intrinsic $\nu_{e}$ level without significantly degrading the signal detection efficiency.

\subsection{Possible experimental approaches}

A traditional challenge in high energy physics experiments has been how to separate out the electromagnetic component in a hadronic jet from the remaining hadrons. In the calorimetric method this was generally achieved by having a high $\mathrm{Z}$ electromagnetic calorimeter in front of the hadron section of the calorimeter. Clearly that technique is not suitable for electron $/ \pi^{0}$ separation. The latter has been traditionally done in open geometry experiments by using a water Cherenkov, a technique adopted by IMB, Kamiokande and SuperKamiokande. The other technology of choice has been the use of low $\mathrm{Z}$ calorimeters (eg CHARM II and BNL oscillation experiment) which allow identification of the electron by tracking.

In principle, at least, in a highly segmented detector electrons can be separated from $\pi^{0}$ 's by utilizing several experimental characteristics:

- finite separation between the vertex and conversion points of the $\gamma$ 's from the $\pi^{0}$,

- two electromagnetic showers (for $\pi^{0}$ ) vs one (for electrons),

- double pulse height right after conversion of a $\gamma$.

Success of the separation based on these criteria requires fine segmentation: longitudinally, less than a radiation length, $X_{0}$; transversely, finer than the 
spatial separation of the two $\gamma^{\prime}$ 's from the $\pi^{0}$ decay. The transverse segmentation also has to be such that individual tracks in the final state can be separated from each other.

Besides the need to distinguish electrons from $\pi^{0}$ 's, one must also distinguish electrons from hadrons. This is harder in a low $\mathrm{Z}$ material and relies on the absence of hadronic interactions (for electrons) and a generally broader track from electrons due to accompanying bremsstrahlung.

The other important characteristic of a good $\nu_{e}$ detector is its energy resolution. The way to reduce the intrinsic $\nu_{e}$ background is to utilize the fact that the events from $\nu_{\mu} \rightarrow \nu_{e}$ oscillations will have a sharp energy spectrum at a predictable energy in contrast to the backgrounds. This is a key feature of the narrow neutrino beam energy spectrum seen by an off-axis detector. $\nu_{e}$ 's from $\mu$ decays will be in roughly the same energy range as $\nu_{\mu}$ 's from $\pi$ decay (and hence oscillated $\nu_{e}$ 's) but have a much broader energy distribution. $K_{e 3}$ decay will typically give much higher energy neutrinos whereas the $\tau$ decay electrons will peak towards low energies. The shape and the level of backgrounds and possible signal are shown in Fig. 5.1.

\subsection{Background measurements and estimates; sys- tematics}

The backgrounds discussed above can be both estimated and measured. We discuss each background in turn.

a) $\nu_{e}$ 's from $\mu$ decay.

The $\nu_{\mu}$ flux at $0^{\circ}$ will be measured in the Near Detector located on axis. Muon flux over all angles can be estimated reliably from those measurements since only kinematics are involved. The $\nu_{e}$ rate and energy distribution from this source can be known with an accuracy of $5 \%$ [30]

b) $\nu_{e}$ 's from $\mathrm{K}$ decay.

This background is about a factor of 5 lower than the one from muon decays. It can be calculated with the basic limitation coming from uncertainties of $\pi / \mathrm{K}$ production cross section by $120 \mathrm{GeV}$ protons. In principle this uncertainty will be reduced by the measurements of the E907 experiment. Most of this background occurs at high $\nu_{e}$ energies, therefore an extrapolation to the region of interest can be performed.

c) Electrons from $\tau$ decays. 


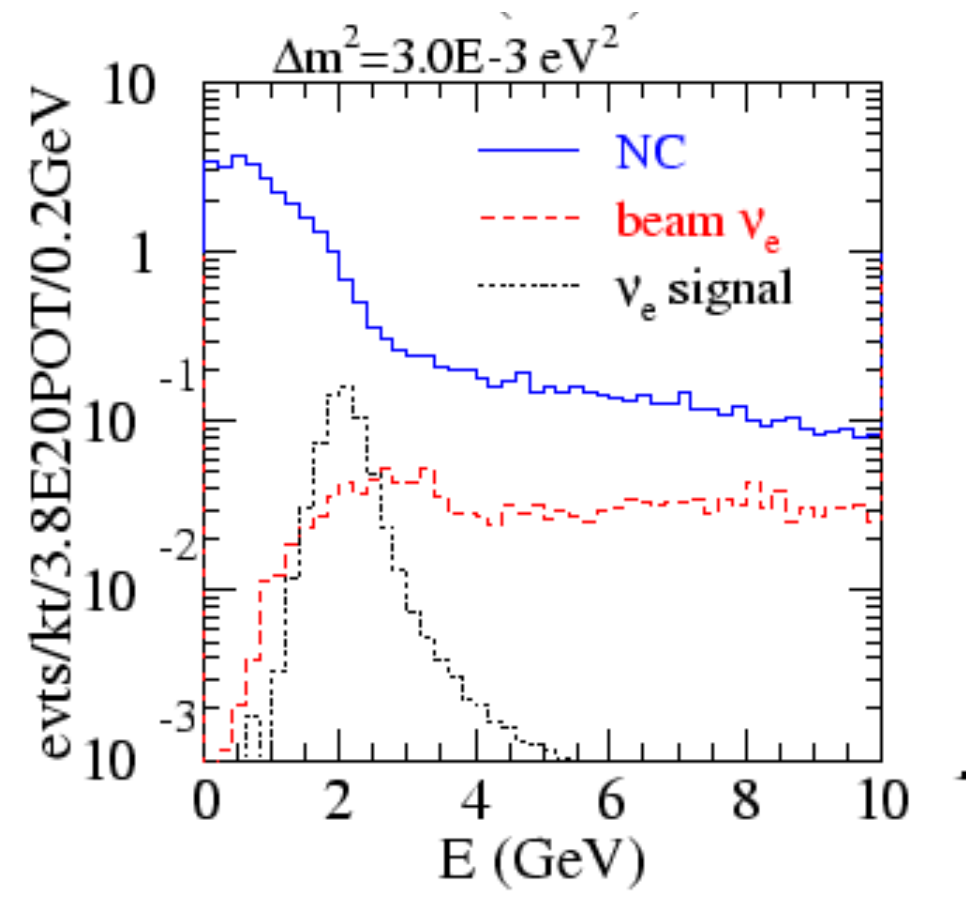

Figure 5.1: Energy distribution of the expected $\nu_{e}$ oscillation signal for $\left|U_{e 3}\right|^{2}=$ 0.01 (black histogram), intrinsic beam $\nu_{e}$ events (red histogram), and observed energy distribution of the $\mathrm{NC}$ events before rejection (blue histogram)

This background is comparable to but somewhat smaller than the one from $\mathrm{K}$ decays in our energy range. The $\nu_{\tau}$ rate can be reliably estimated from oscillation parameters and $\nu_{\mu}$ flux. There is some uncertainty in the production cross section and angular distributions but, because of the low overall rate, these uncertainties will have a negligible contribution to the overall systematic uncertainty on the total background.

d) $\mathrm{NC}$ events misidentified as $\nu_{e}$ interactions.

An a priori estimate of this background is somewhat more difficult than for the cases discussed above and it will need to be measured. Initial Monte Carlo calculations indicate that its contribution can be reduced to a level of the intrinsic $\nu_{e}$ background or better while maintaining the efficiency for $\nu_{e}$ identification at about $30-40 \%$. This source of background will probably give the dominant contribution to the systematic error.

A measurement of the NC background can be made using a near off-axis detector located at an angle similar to the one of the far detector. Such a detector is possible in the bypass tunnel downstream of the NuMI absorber 
where the neutrino spectrum would be similar to that in the far detector.

e) $\nu_{\mu} \mathrm{CC}$ events mis-identified as $\nu_{e}$ interactions.

Such a misidentification would require a failure to detect the muon, residual hadronic energy in the range of the expected oscillation signal, and misidentification of a pion as an electron. We note that the rate of the $\nu_{\mu}$ $\mathrm{CC}$ events is significantly reduced by the oscillations. Initial estimates indicate that this background is significantly lower than the NC background.

f) Hadrons misidentified as electrons.

There is now a significant amount of data on electron-muon (or pion) separation in water Cherenkov detectors with the conclusion that this background is insignificant for energies above few hundred MeV. A low $\mathrm{Z}$ detector would have to be exposed to hadrons and electrons in a test beam. Such an exposure will guide the design of the detector and allow one to develop an optimum algorithm for e/hadron separation.

In summary, the Monte Carlo studies to date indicate that for a detector in the tens of kton range the systematics associated with imperfect knowledge of the background levels can be kept sufficiently low so that the dominant uncertainty will be due to statistical fluctuations. More detailed studies with the detector of choice are needed, however, to confirm up this initial impression.

\subsection{Physics Potential of the NuMI Off-axis Beam}

At the present time there are a large number of unanswered questions in neutrino physics. Some of the most important ones that are relevant to the proposed program are:

- Is the LSND effect related to neutrino oscillations?

- Is the LMA solution to the solar neutrino problem the correct one?

- What is the value of $\theta_{13}$ ?

- What is the sign of the mass hierarchy?

- Is CP conserved in the neutrino sector?

- Is CPT conserved in the neutrino sector? 
It is highly likely that the first two questions will be answered by the time the experimental program described here will begin. The last 4 topics are the focus of the proposed program.

Our discussion below tacitly assumes that the answer to the first question is in the negative. If that assumption is incorrect, the neutrino community will have to rethink seriously the future program.

Regarding the second question, KamLAND should provide a definitive answer here should they see depletion of events. If they do not, and future NC data from SNO reinforce the support for the LMA solution, then very likely new and unexpected physics lurks in the neutrino sector. The argument for new experimental efforts becomes even stronger.

The experimental program being proposed here takes into account the need to maintain maximum flexibility as answers to the last four questions become slowly elucidated. We divide our program into two phases: the second phase will build on the results from Phase I as well as on the information provided by the potential JHF program. We can, however, start planning Phase I now.

At the present time two generic site possibilities appear to be front runners for the Phase I of the proposed experiment. The first one is at $712 \mathrm{~km}$, near the current Soudan experiment. The second site possibility is in the region in Canada between the southern and northern branches of the Trans Canadian Highway, between 850 and $980 \mathrm{~km}$. The near-Soudan site gives good sensitivity to $\nu_{e}$ appearance signal, on the assumption of $\Delta m_{32}^{2}=3 \times 10^{-3} \mathrm{eV}^{2}$. The further sites are better if $\Delta m^{2}$ is lower and offer larger matter and CP violation effects and thus a chance to obtain at least the first glance at these two phenomena.

The sensitivity of Phase I to observation of $\nu_{e}$ 's is hard to quantify because the size of the expected signal depends not only on the value of $\theta_{13}$ but also on the pattern of mass hierarchy (through matter effects) and the value of the $\mathrm{CP}$ violation phase as shown in Fig. 5.2. At low values of $\sin ^{2} 2 \theta_{13}$ there is an additional potentially important contribution to the uncertainties in the interpretation of the experimental results due to the uncertainty of the value of $\Delta m_{12}^{2}$. We try to give a general estimate by making a rough comparison with the JHF[20] proposal (see below, Tables 5.1 and 5.2). In Table 5.2 we have assumed a $20 \mathrm{kt}$ detector, $5 \mathrm{yr}$ experiment, $85 \%$ fiducial volume, $\mathrm{NC}$ background equal to the beam $\nu_{e}$ background and $4 \times 10^{20}$ protons delivered per year and no matter or CP effects. Because the matter effects are much more important at NuMI distances, NuMI sensitivity could increase by up to $30 \%$ at $712 \mathrm{~km}$ for normal mass hierarchy, or more at $900 \mathrm{~km}$. 

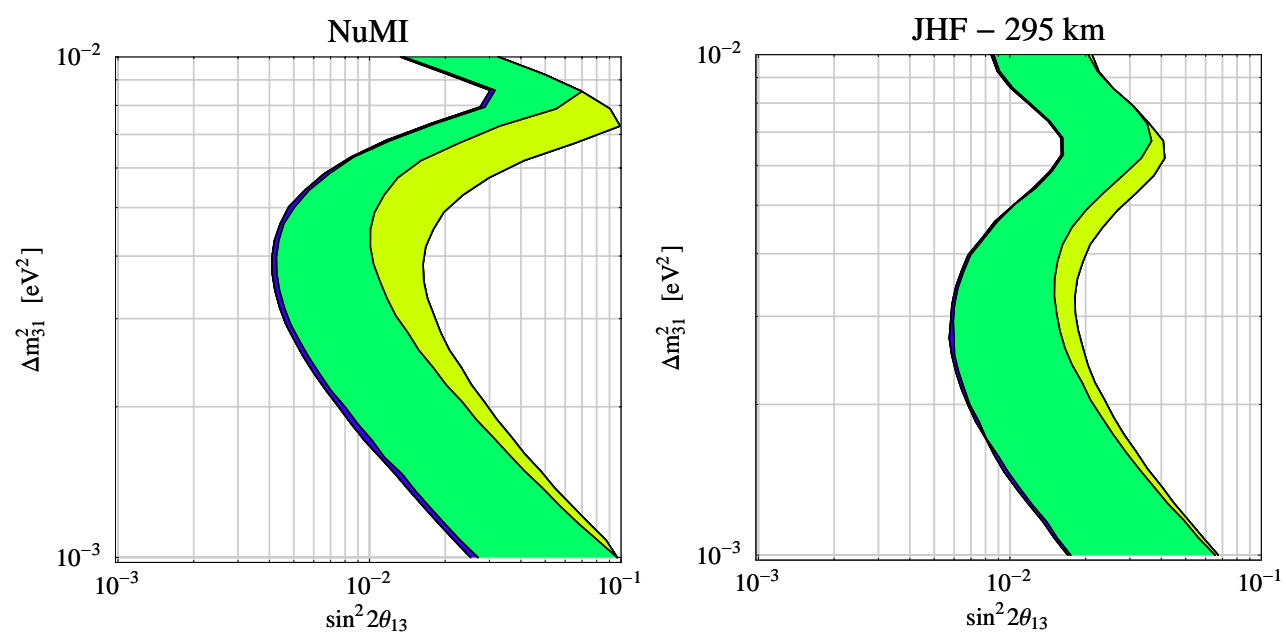

Figure 5.2: Interpretation of the observed $\nu_{\mu} \rightarrow \nu_{e}$ transition probability in terms of $\sin ^{2} 2 \theta_{13}$ for the $\operatorname{NuMI}(712 \mathrm{~km})$, left, and the JHF (right). The left edge is the limit implied by the statistical errors. The right edge of the blue region corresponds to a $10 \%$ error on the background subtraction. The green band represents uncertainty due to the value of $\delta$. The yellow band shows the impact of degeneracy between $\theta_{23}<\pi / 4$ and $\theta_{23}>\pi / 4$ solutions[31].

Fig. 5.3 shows the CP trajectory contours in the bi-probability space for $\nu_{e}$ and $\bar{\nu}_{e}$ appearance at $712 \mathrm{~km}$ and $950 \mathrm{~km}$ using optimized energies at those distances. We use the oscillation parameters of the solar neutrinos $\sin ^{2} 2 \theta_{12}=0.8$, $\Delta m_{12}^{2}=5 \times 10^{-5} \mathrm{eV}^{2}$ and values of $\sin ^{2} 2 \theta_{13}$ corresponding to an oscillation probability equal to $1 / 2$ and $1 / 5$ of the current CHOOZ limit. It is clear that given sufficient statistics the proposed experimental program would be very well positioned to identify the sign of the matter effect and obtain some information on $\mathrm{CP}$ violation, especially when one combines information from neutrino and antineutrino running. For comparison we show also the same contour plots, for identical oscillation parameters, for the proposed JHF program with $L=295 \mathrm{~km}$ and $E_{\nu}=0.8 \mathrm{GeV}$.

Studies of energy dependence of the disappearance of $\nu_{\mu}$ CC events can provide information about equivalence of $\Delta m_{32}^{2}$ for $\nu_{\mu}$ and $\bar{\nu}_{\mu}$ oscillations and thus validity of $\mathrm{CPT}$ in the neutrino sector. This comparative measurement will probably be dominated by systematic effects; no quantitative estimates of how well one can do have been performed to date but $1 \%$ accuracy does appear possible. 

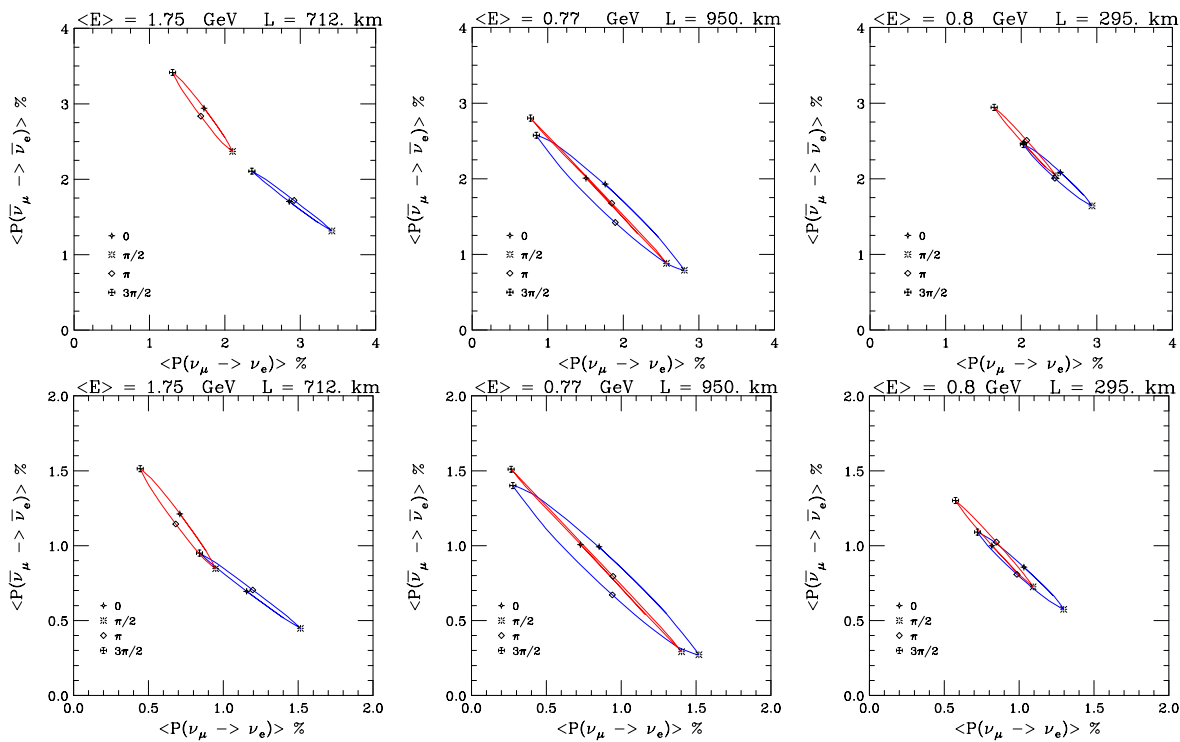

Figure 5.3: CP trajectory contours in the bi-probability space for $\nu_{e}$ and $\bar{\nu}_{e}$ appearance at $295 \mathrm{~km}, 712 \mathrm{~km}$ and $950 \mathrm{~km}$. Top row is for $\sin ^{2} 2 \theta_{13}=0.05$, bottom row is for $\sin ^{2} 2 \theta_{13}=0.02$. Symbols denote the expected values of probabilities for specific values of the CP phase.

\subsection{Comparison with JHF Phase I}

The phase I of the contemplated JHF program would utilize the proposed neutrino beam from the JHF accelerator, currently under construction, and the SuperKamiokande detector, to be reconstructed after the end of the K2K experiment. The source to the detector distance would be $295 \mathrm{~km}$ and the mean energy for the currently favored $2^{\circ}$ off-axis beam is $0.8 \mathrm{GeV}$, thus the $\mathrm{L} / \mathrm{E}$ ratio would be quite comparable to the value in the proposed NuMI program.

Similarly to the NuMI program it plans to achieve better than a factor of 10 improvement in $\sin ^{2} 2 \theta_{13}$ over the CHOOZ limit at a value of $\Delta m_{32}^{2}=3 \times 10^{-3}$, somewhat better at lower values and somewhat worse at higher values. The matter effects are much smaller than in NuMI case and it probably will not be possible to obtain any significant information on $\mathrm{CP}$ violation in this Phase. The most recent proposal does not indicate any plans to run in the $\bar{\nu}_{\mu}$ mode.

The sensitivity of the proposed JHF PhaseI to matter effects and CP violation is quantified in Fig. 5.3. The relative sensitivity of the two proposed programs to the $\nu_{e}$ appearance signal are compared in Tables 5.1 and 5.2. We use the same 


\begin{tabular}{|c|c|c|c|c|}
\hline & $\nu_{\mu} \mathrm{CC}$ & $\mathrm{NC}$ & Beam $\nu_{e}$ & Signal $\nu_{e}$ \\
\hline all & 10714 & 4080 & 292 & 302 \\
\hline after cuts & 1.8 & 9.3 & 11 & 123 \\
\hline
\end{tabular}

Table 5.1: Anticipated number of signal and background events in JHF OAB 2 deg beam

\begin{tabular}{|c|c|c|c|c|}
\hline & $\nu_{\mu} \mathrm{CC}$ & $\mathrm{NC}$ & Beam $\nu_{e}$ & Signal $\nu_{e}$ \\
\hline all & 12104 & 5696 & 295.4 & 293 \\
\hline after cuts & & 10.2 & 10.2 & 85.5 \\
\hline
\end{tabular}

Table 5.2: Anticipated number of signal and background events in the detector located $9 \mathrm{~km}$ off-axis at a distance of $712 \mathrm{~km}$ from Fermilab

parameters $\left(\Delta m_{32}^{2}=3 \times 10^{-3}\right.$ and $\sin ^{2} 2 \theta_{13}=0.1$, i.e. at the CHOOZ limit, and a 5 yr exposure for both proposed programs). We assume $4 \times 10^{20} \mathrm{POT} / \mathrm{yr}$ for NuMI and a $20 \mathrm{kt}$ detector with $85 \%$ fiducial volume. We use numbers from Table 2 of the JHF proposal. 


\section{Chapter 6}

\section{Future Evolution of the Off-Axis Neutrino Program}

In addition to searching for unexpected phenomena, such as CPT violation, the off-axis program strives to measure $\sin ^{2}\left(2 \theta_{13}\right)$, the order of the mass hierarchy, and the CP-violating phase $\delta$. It is rather unlikely that the Phase I program described in this proposal will be able to complete these objectives. In almost all scenarios there will be a strong argument to enhance the program by increasing the flux times detector mass and possibly by moving detectors or building additional detectors to obtain different values of $L$ and $E$.

There are three reasons for expecting the need to eventually expand the program:

a) The effects, particularly those due to $\mathrm{CP}$ violation, are quite likely be small, requiring greatly increased statistics. A reasonable expectation is that one would like to eventually increase the proton flux by a factor of four, as envisioned in the Proton Driver study, and to increase the detector mass by a factor of five.

b) Since the parameters scale differently with $L$ and $E$, different detector locations will be advantageous to measure them.

c) Different detector locations might be necessary to resolve ambiguities that can occur with a single detector location.

We will give a few examples to illustrate these points. The first goal of the Phase 
I program is to detect a positive signal for $\nu_{\mu} \rightarrow \nu_{e}$ oscillations. Such a signal should be seen if $\sin ^{2}\left(2 \theta_{13}\right)$ is greater than 0.01 . If Phase I is successful in this, then we can proceed to try to determine the parameters. If no significant signal is seen, then increased flux times detector mass will be necessary to continue the search for a non-zero value of $\sin ^{2}\left(2 \theta_{13}\right)$. Systematic errors need to be considered carefully to determine what additional subsidiary experiments are needed to control them.

Detection of a signal for $\nu_{\mu} \rightarrow \nu_{e}$ appearance will not in itself provide a measurement of $\sin ^{2}\left(2 \theta_{13}\right)$ since relatively large matter effects introduce a two-fold ambiguity in the measurement of $\sin ^{2}\left(2 \theta_{13}\right)$, in the absence of $\mathrm{CP}$-violating effects. In the presence of $\mathrm{CP}$-violating effects, there will be additional ambiguities caused by the value of $\delta$, unless the value of $\sin ^{2}\left(2 \theta_{13}\right)$ is relatively large, greater than about 0.02 .

To understand how we proceed to resolve ambiguities and measure the parameters, it is useful to review how these effects scale with distance, energy, and the small parameters. Since both effects are maximal at the peaks of the $\Delta m_{13}^{2} \approx$ $\Delta m_{23}^{2}$ oscillation, we assume that measurements will be done at odd-integer values of the oscillation phase. The practical values of $n \pi / 2$ are the first peak, $n=1$, and the second peak, $n=3$. This implies the a scaling in $E$ is equivalent to a scaling in $L / n$. In the NuMI region, the matter effects scale as $\theta_{13}^{2} E$ or $\theta_{13}^{2} L / n$. CP violating effects scale as $\theta_{13} \Delta m_{12}^{2} n$.

Consider the case of relatively large values of $\theta_{13}$ first. The matter effects, which scale as $\theta_{13}^{2}$ dominate the $\mathrm{CP}$-violating effects, which scale as $\theta_{13}$. In this regime, the mass hierarchy can be determined without ambiguity by the combination of neutrino and antineutrino runs. (See Fig. 1.2) For NuMI baselines, the matter effects are large. The double ratio

$$
\left[\frac{\sigma\left(\nu_{\mu} \rightarrow \nu_{e}\right)}{\sigma\left(\bar{\nu}_{\mu} \rightarrow \bar{\nu}_{e}\right)}\right]_{\text {normal }} /\left[\frac{\sigma\left(\nu_{\mu} \rightarrow \nu_{e}\right)}{\sigma\left(\bar{\nu}_{\mu} \rightarrow \bar{\nu}_{e}\right)}\right]_{\text {inverted }}
$$

is 1.7 for $L=712 \mathrm{~km}, 1.8$ for $L=850 \mathrm{~km}$, and 2.0 for $L=985 \mathrm{~km}$. Thus, once electron appearance has been seen, the value of $\theta_{13}$ and the mass hierarchy can be determined simultaneously. However, the necessary antineutrino running requires increased integrated flux times detector mass, since fewer antineutrinos are produced and their cross section is lower.

If $\sin ^{2}\left(2 \theta_{13}\right)<0.02$, then there can be ambiguities caused by the CP-violating phase $\delta$. To resolve these ambiguities, and to make a measurement of $\delta$ regardless of the value of $\sin ^{2}\left(2 \theta_{13}\right)$, it may be desirable to make measurements at the second oscillation maximum, $n=3$. The scaling laws indicate that at the second maximum the matter effects decrease by a factor of three and the CPviolating effects increase by a factor of three. (There is however, a decrease in both the flux (see Fig. 4.3 and the cross section, due to the reduced neutrino 
energy.) Since, in the off-axis beam, each detector location represents a single $L$ and a single $E$, this would require an additional far detector site. Water Cherenkov detectors might be more appropriate for the low energies (about 500 $\mathrm{MeV}$ ) of the second maximum. They have the great advantage that the cost of detectors scales as the area of the detector rather than as the mass.

Finally, it should be noted that there is a high degree of complementarity between a JHF program at $295 \mathrm{~km}$, and NuMI off-axis detectors at $712 \mathrm{~km}$ (first maximum) and $985 \mathrm{~km}$ (second maximum) as shown in the following table.

\begin{tabular}{|c|c|c|c|c|}
\hline Detector & $\begin{array}{c}L \\
(\mathrm{~km})\end{array}$ & $\begin{array}{c}E \\
(\mathrm{GeV})\end{array}$ & $\begin{array}{c}\text { Relative } \\
\text { matter effect }\end{array}$ & $\begin{array}{c}\text { Relative } \\
\text { CP effect }\end{array}$ \\
\hline \hline JHF & 295 & 0.6 & 1.0 & 1.0 \\
\hline NuMI Phase I & 712 & 1.4 & 2.9 & 1.0 \\
\hline NuMI Phase II & 985 & 0.7 & 1.1 & 3.0 \\
\hline
\end{tabular}

Measurements at both a $300 \mathrm{~km}$ and a 700 to $1000 \mathrm{~km}$ baseline will further aid in resolving ambiguities and checking consistency with the theory.

In summary, we expect that the investigation of the neutrino mass hierarchy and the parameters of the MNS matrix will be an extended and fruitful program. Arguably it is as or more important than the efforts now underway to measure the parameters and check the consistency of the CKM matrix, and should receive, with time, at least a similar level of support. We expect that it will be a major component of the Fermilab program in the LHC era. 


\section{Chapter 7}

\section{Detector}

An off-axis NuMI neutrino beam offers an unique opportunity to study $\nu_{\mu} \rightarrow \nu_{e}$ oscillations. There will be a very large number of $\nu_{\mu}$ 's oscillating away. Most of the resulting $\nu_{\tau}$ 's will be below the kinematical threshold for $\tau$ production hence a small admixture of $\nu_{e}$ 's should be detectable with as small background as possible.

To take full advantage of this opportunity it is necessary to construct a new detector capable of the detection and identification of the $\nu_{e}$ charged current interactions. Such a detector must meet several challenges:

- it must have fine granularity in order to identify the final state electrons

- it must have very large mass to provide maximal sensitivity to the oscillation amplitude

- it must have an acceptable cost per unit mass

- it must be able to operate on surface or under a small overburden, as there are no convenient underground locations

The detector should be optimized for the neutrino energy range of $1-3 \mathrm{GeV}$.

The ultimate sensitivity of the experiment, at the $90 \%$ C.L. will be determined by the condition $S \geq 1.26 \sqrt{B}$, where $S=\epsilon \times \#$ of signal events and $B=$ $\epsilon \times \#$ of $\nu_{e}+\eta \times N C$ with $\epsilon$ being the efficiency for the detection and identification of the $\nu_{e}$ interactions and $\eta$ being the mis-identification probability for $\mathrm{NC}$ interactions. The sensitivity of the experiment improves with the square root 
of the exposure time until it becomes limited by the the systematic error of the background. The latter is likely to be dominated by the error on the NC component of the background.

There appear to be three classes of a possible new detector:

- water Cherenkov. It is likely to offer the biggest advantage in terms of the mass of the detector but in the energy regime of interest at NuMI it appears to be dominated by the NC background, i.e. $\eta \times N C \gg \epsilon \times \nu_{e}$. The efficiency for the signal events is likely to be around $25 \%$.

- fine grained calorimeter. This class of detectors is characterized by the signal detection efficiency of the order of $35-40 \%$ while maintaining a balance between the intrinsic background and the NC-induced one, i.e. $\eta \times N C \sim \epsilon \times \nu_{e}$

- Liquid Argon TPC. This is probably the most challenging, but the most powerful detector, offering the highest signal efficiency $\epsilon \sim 90 \%$ and background level at the intrinsic $\nu_{e}$ component, $\eta \times N C \ll \epsilon \times \nu_{e}$

The NuMI neutrino beam will start operations in the early 2005. It is highly desirable that the Phase I of the proposed program is implemented as early as it is practically possible to yield the initial information on the $\nu_{e}$ appearance and to provide guidance for the future phases of the program. Such an accelerated time scale leaves no time for the extensive detector R\&D program, but fortunately none is necessary. Forty years of experience in constructing neutrino detectors combined with the impressive progress in the detector technology in the past decade offers several possible implementations for a detector of the 20 kton class.

Phase II of the program may require much large detector, perhaps in the range of 100-200 ktons. It may well be that the the future detector may be required to operate at the energies below $1 \mathrm{GeV}$, if the second oscillation maximum will offer the best sensitivity. It is not clear, at the present time, if the detector technology for the Phase II will be necessarily the same as the Phase I detector.

Studies of neutrino oscillations, especially in Phase II may require optimization of the detector location. Given the cost of the initial investment it may be advantageous to have a detector design allowing the relocation of the detector from one location to another, or, perhaps, reconfiguration of the detector into two or more smaller detectors located in different positions.

In Appendix A we describe several alternatives for a possible new detector to demonstrate that there are realistic conservative designs of affordable detectors as well as there are possible new alternatives. 


\section{Chapter 8}

\section{Detector Sites, Physics Considerations}

Progress in neutrino physics worldwide will be optimized if the future NuMI program is comparable in quality and as complementary as possible to the planned JHF program. The interpretation of any observation of $\nu_{e}$ 's due to oscillations will be difficult because of simultaneous contributions of $\theta_{13}$, matter effects, and $\mathrm{CP}$ phase, $\delta$. Several measurements with different conditions will be necessary to unravel the situation.

For a given baseline distance, $L$, different contributions to the $\nu_{\mu} \rightarrow \nu_{e}$ oscillation probability are principally a function of $L / E$. In our region of parameter space a contribution of matter effects grows with the distance $L$. The baseline in the JHF program is constrained to be relatively short, i.e. $295 \mathrm{~km}$. Thus the natural choice for NuMI would be to have as long a baseline as is consistent with the energies of Main Injector beams and availability of satisfactory sites.

It is convenient to discuss optimization of the detector position in terms of the oscillation phase $\phi=1.27 L \Delta m_{32}^{2} / E$. Generally, unless there are strong cancellations from different contributions, the maximum $\nu_{e}$ appearance signal will be obtained when $\phi=\pi / 2$. Due to $1 / L^{2}$ dependence of the signal $S$ and the background $B$, the optimum sensitivity, taken as a ratio of $S / \sqrt{B}$, will correspond to a distance L about $25 \%$ shorter. For $\Delta m_{32}^{2}=0.3 \mathrm{eV}^{2}$, this translates to an optimum transverse distance away from the beam axis of about $9 \mathrm{~km}$ (independent of $L$ ). This distance scales inversely with $\Delta m_{32}^{2}$.

The maximum matter effects occur at a larger value of the phase which trans- 


\begin{tabular}{|c|c|c|c|c|}
\hline Distance & 0.07 - matter & $0.07 \mathrm{CPV}$ & 0.1 - matter & $0.1 \mathrm{CPV}$ \\
\hline $295 \mathrm{~km}$ & 0.56 & 2.87 & 1.14 & 4.08 \\
\hline $712 \mathrm{~km}$ & 2.45 & 4.92 & 4.97 & 6.99 \\
\hline $848 \mathrm{~km}$ & 3.01 & 5.08 & 6.07 & 7.21 \\
\hline $985 \mathrm{~km}$ & 4.50 & 5.76 & 9.12 & 8.18 \\
\hline
\end{tabular}

Table 8.1: Potential contributions, in parts per thousands, to oscillation probability from matter effects and $\mathrm{CP}$ violation

lates into an equivalent transverse distance of $13-15 \mathrm{~km}$. In addition, as mentioned above, they grow with $\mathrm{L}$. CP violation effects also generally increase with increasing $\phi$. The NuMI neutrino beam comes to the surface at the distance $L=747 \mathrm{~km}$ from Fermilab. A transverse displacement from the beam axis can be achieved either by going further or closer, along the nominal beam direction, effectively taking the off-axis beam in the vertical plane or by going sideways at a baseline corresponding to the nominal $0^{\circ}$ distance, or a combination of both.

An important issue is the change in sensitivity as we move away from the optimum distance. This defines how precisely we need to know $\Delta m_{32}^{2}$ before choosing the site. This change is relatively small if we try to optimize the size of the total appearance signal, and a transverse displacement from the optimum (at a fixed L) can be as large as $\pm 2 \mathrm{~km}$. The sensitivity is greater (by roughly a factor of 2) if one tries to maximize a contribution of the matter effects. These comments should be viewed as qualitative statements since due to the coherent nature of all contributions, a more rigorous statement can be made only in the context of specifying values of all the relevant parameters.

Another issue which needs to be considered is the variation of total $\nu_{\mu}$ flux as a function of distance $L$ and mean beam energy, controlled by the distance from the beam axis. The flux will fall off with $L$ as $1 / L^{2}$. For $120 \mathrm{GeV}$ proton energy and off-axis angles corresponding to neutrino energy range $1-2 \mathrm{GeV}$, the flux times cross section drops a little faster than $E^{2}$. Thus we gain slightly in event rate, for the same phase $\phi$, as we go to larger distances, but that effect is sufficiently small so as not to be very important.

To give an idea of the relative magnitude of potential contributions due to matter effects and CP violation in different sites, we present those contributions, in parts per thousand addition to the $\nu_{e}$ appearance probability, in Table 8.1. We show them for two values of $\theta_{13}, 0.07$ and 0.1 , corresponding roughly to a factor of 5 and 2.5 below the current CHOOZ limit, and for $\Delta m_{32}^{2}=2.5 \times 10^{-3} \mathrm{eV}^{2}$, the currently best fit to the K2K and SuperKamiokande data. 


\section{Chapter 9}

\section{Cost and Schedule, Milestones}

\subsection{Cost}

Given the status of the project it is not possible to have a credible cost estimate. It appears, though, that the main cost driver will be the construction of the active detectors. There are several possible detector designs using mature and well understood technologies, thus making a cost of the active detectors reasonably well predictable. These costs are in the range of $\$ 15-20 M$ for a 20 kton class detector. Engineering issues associated with construction of a very large and massive detector are very challenging. Costs associated with the construction of the absorber, support structures, experimental halls etc. cannot be very well known before the engineering studies are completed. We aim at the design of the Phase I of the experiment for a cost not exceeding $\$ 50-100 M$.

The second phase of the program may involve upgrades to the accelerator complex of Fermilab. These upgrades are subject of a dedicated studies [32]. It is likely that the Phase II detectors will require a significant increase in the fiducial mass (a factor of 5 or so) and, perhaps, in the detector capabilities. It is not possible to give a reliable cost estimate of these detectors, but it appears likely that this phase will require investment of the order of $\$ 250-300 M$. 


\subsection{Schedule}

NuMI beam line will start delivering neutrinos, on- and off-axis, at the beginning of the calendar year 2005. While it is not possible to complete a construction of a significant fraction of a large detector in such a short time frame, it is important to point out that a timely construction of a new detector would maximize the scientific output of the NuMI facility. It is important to note that a modular detector design may enable a start of the data taking long before the coentruction is completed.

Proposed Phase I detectors utilize off-the-shelf technology, not requiring timeconsuming $\mathrm{R} \& \mathrm{D}$ process. Detailed engineering studies are necessary, though, before the final approval of the experiment. Modular design of the experiment may enable parallel construction at several locations, it may simplify the final assembly as well.

It appears, therefore, that the schedule of a possible experiment will be driven by the approval process and by the availability of the construction funds. We hope for an approval process to proceed efficiently enough to provide construction funds starting in FY2005. We also hope that the construction of the detector may start earlier through forward-funding from universities and/or from foreign sources.

\subsection{Milestones}

2002 Summer submission of a Letter of Intent

2002 Fall/winter detector design work, measurement of the cosmic ray background

2003 Spring experimental verification of the level of cosmic ray-induced background

2003 Summer Proposal to the Fermilab PAC, NSF

2003-2004 construction of prototypes,

2003 Fall Far site selection

2004 Summer electron and hadron test beam studies of the detector prototypes

2004 Reviews, validation,

2004 Summer final approval 
2004 Fall start construction of the far detector building and facility

2005 Construction and installation of the Near Off-Axis detector

2005 Summer Far detector building complete

2005 Fall Near off-axis detector starts taking data

2005-2006-2007 construction of the Far Off-axis detector

2006 Summer start taking data with the initial 5 kton detector

2007-2012 Phase I of the program

We envisage that the Phase II of the program will require an increased useful mass of the neutrino detector by a factor of five or so. It can be achieved through construction of more detector modules of the same design as in the Phase I or by a construction of a diferent detector with much higher detection efficiency (like Liquid Argon TPC) or much larger mass (like water Cherenkov). To be in a position to select the most apropriate strategy for the Phase II it is imperative that adequate funding for the required $R \& D$ process is available as early as possible. 


\section{Acknowledgments}

Many people have contributed to the ideas and concepts presenetd here. Their contributions are gratefully acknowledged.

Special thanks go to Friederich Dydak for his contribution to the chapter on the evolution of neutrino physics.

Achim Geisser, Tommaso Tabarelli de Fatis and Carlo Gustavino were the source of inspiration for the LoDen detector concept.

Dan Marlow was a source of advice on the RPC chambers and has contributed a section on the gas and HV systems.

Marc Mengel made it possible to overcome all annoying problems with forcing Postscript files into a Latex document. 


\section{Bibliography}

[1] Kamiokande Collaboration, Y. Fukuda et al., Phys. Lett. B335 (1994) 237; Super-Kamiokande Collaboration, Y. Fukuda et al., Phys. Rev. Lett. 81 (1998) 1562; S. Fukuda et al., ibid. 85 (2000) 3999.

[2] CHOOZ collaboration, M. Apollonio et al., Phys. Lett. B 466415.

[3] SNO Collaboration, Q. R. Ahmad et al., Phys. Rev. Lett. 87 (2001) 071301.

[4] LSND Collaboration, C. Athanassopoulos at al., Phys. Rev. C 55, (1997) 2079

[5] E. Church et al. [BooNe Collaboration], Booster: BooNE," FERMILAB-P0898.

[6] V. Barger, S. Geer, R. Raja, K. Whisnant, Phys. Rev. D63, 113011 (2001), I. Mocioiu and R. Shrock, JHEP 0110, 050 (2001)

[7] H. Minakata and H. Nunokawa, JHEP 0110 (2001) 001 [hep-ph/0108085].

[8] K2K Collaboration, S. H. Ahn et al., Phys. Lett. B 511 (2001) 178

[9] S. Fukuda et al. [Super-Kamiokande Collaboration], Phys. Rev. Lett. 85, 3999 (2000) [arXiv:hep-ex/0009001].

[10] The NuMI Facility, Technical Design Report, Version 1.0, October 1998

[11] The MINOS Detectors, Technical Design Report, NuMI-L-337, October 1998

[12] IMB Collaboration, R. Becker-Szendy et al Phys. Rev. Lett. 66 (1991) 2561

[13] Y. Fukuda et al, Phys. Lett. B 335 (1994) 237 
[14] M. Diwan, M. Messier, B. Viren, L. Wai, A study of $\nu_{\mu} \rightarrow \nu_{e}$ sensitivity in MINOS, NuMI-NOTE-SIM-0714

D. Petyt, Nu-mu to Nu-e in MINOS, NuMI-NOTE-SIM-0576

A. Para, Electron ID and transverse granularity, NuMI-NOTE-SIM-0284

[15] Q. R. Ahmad et al. [SNO Collaboration], arXiv:nucl-ex/0204008.

[16] L. De Braeckeleer [KamLAND Collaboration], Nucl. Phys. Proc. Suppl. 87, 312 (2000).

[17] S. Bonetti et al. [Borexino Collaboration], Nucl. Phys. Proc. Suppl. 28A, 486 (1992).

[18] Expression of Interest in an experiment at the CNGS to measure $\theta_{13}$, http://home.cern.ch/dydak/oscexp.ps

[19] K. Elsener et al, The CERN Neutrino beam to Gran Sasso, CERN 98-02, INFN/AE-98/05

[20] Y. Itow et al, The JHF-Kamioka Project, hep-ex/0106019

[21] J.J. Gomez Cadenaz et al Physics Potential of Very Intense Conventional Neutrino Beams, hep-ph/0105297

M. Vretenar, A high-intensity $\mathrm{H}^{-}$Linac at CERN based on LEP-2 ccavities, CERN/PS 2000-059 (NUFACT Note 040)

[22] P. Zucchelli, CERN-EP/2001-056, hep-ex/0107221

[23] there is a huge number of papers on neutrino factories covering the machine and experimental aspects as well as the physics potential. A dedicated series of conferences NuFactXX is covering the progress of the project. Proceedings of these conferences and the references cited therein give a good indication of the amount of effort devoted.

[24] F. Boehm et al., Phys. Rev. D 64, 112001 (2001) [arXiv:hep-ex/0107009].

[25] Y. Kozlov, L. Mikaelyan and V. Sinev, hep-ph/0109277

[26] M. Kostin et al Proposal for Continuously-Variable Beam Energy, NuMINOTE-BEAM-0783

[27] D. Beavis et al, Proposal of BNL AGS E-889 (1995)

[28] A. Para, M. Szleper, Neutrino Oscillation Experiments using Off-axis NuMI Beam, hep-ex/0110032

[29] M. Szleper, A. Para, Neutrino Spectrum at the Far Detector, hep$\mathrm{ex} / 0110001$

[30] M. Szleper, talk at the New Initiatives for the NuMI Neutrino Beam, May 2002 , 
[31] P. Huber, M. Lindner, W. Winter, TUM-HEP 464/02

[32] G. Barenboim et al, report in preparation

[33] the BELLE KLM detector group, Nuclear Instruments and Methods in Physics Research A 449 (2000) 112-124

see also Nuclear Instruments and Methods in Physics Research A 456 (2001) 109-112

[34] C. Gustavino et al. A glass resistive plate chambers for large experiments, Nuclear Instruments and Methods in Physics Research A 457 (2001) 558-563

[35] C. Hagner, presentation at the New Initiatives for the NuMI Neutrino Beam workshop.

[36] N. Y. Agafonova et al. [MONOLITH Collaboration], LNGS-P26-2000.

[37] Border et al, NIM A 463, 194-204 (2001)

[38] Particle Data Group

[39] W. N. Hess et al., (Phys. Rev 116,445(1959)

[40] D. H. Perkins, Soudan 2 Internal Note; PDK-445 (1990)

[41] K.Ruddick, 25th Int.C.R. Conf, Durban (1997)

[42] M. Messier, talk at the New Initiatives for the NuMI Neutrino Beam workshop, May 2002

[43] C. Mauger presentation at the NuInt2001 workshop, KEK, Dec. 2001 http://neutrino.kek.jp/nuint01/

[44] C. Rubbia, The Liquid Argon Time Projection Chamber: a New Concept for Neutrino Detector, CERN-EP/77-08 (1977).

[45] ICARUS home page: http://www.aquila.infn.it/icarus/

[46] The ICARUS Collaboration, Cloning of T600 Modules to Reach the Design Sensitive Mass, ICARUS-TM/01-09, http://pcnometh4.cern.ch/Proposals/t2400doc_lowres.pdf

[47] D.B. Cline, F. Sergiampietri, J.G. Learned, K.T. McDonald, LANNDD, A Massive Liquid Argon Detector for Proton Decay, Supernova and Solar Neutrino Studies, and a Neutrino Factory Detector (May 24, 2001), astro$\mathrm{ph} / 0105442$

[48] F. Sergiampietri, On the Possibility to Extrapolate Liquid Argon Technology to a Supermassive Detector for a Future Neutrino Factory, presented at NuFACT'01 (May 26, 2001), http://www.hep.princeton.edu/ñcdonald/nufact/sergiampietri_nufact01.pdf 
[49] F. Cavanna, O. Palamara for the ICARUS Collaboration, Possible Application of the ICARUS technology for Studies of Neutrino Interactions in the Intermediate Energy Range, ICARUS-TM/02-02,

http://www.aquila.infn.it/icarus/icarus_tm/icarus_02_02_NuInt01.pdf

[50] D. Harris, Comparison of Different Detectors with Same Beam, (Jan. 18, 2002),

http://muonstoragerings.cern.ch/NuWorkshop02/presentations/harris.pdf

[51] A. Bueno et al., Nucleon decay searches: study of nuclear effects and background, ICARUS-TM/01-04 (Oct. 9, 2001),

http://www.aquila.infn.it/icarus/icarus_tm/icarus_01_04.pdf

[52] Among a vast literature, see, for example, J.C. Pati, With Grand Unification Signals in, Can Proton Decay be Far Behind? (June 7, 2001), hep$\mathrm{ph} / 0106082$.

[53] See sec. 6.8 of [46].

[54] S. Ozaki et al., eds., Feasibility Study-II of a Muon-Based Neutrino Source (June 14, 2001), http://www.cap.bnl.gov/mumu/studyii/FS2-report.html

[55] A. Bueno, M. Campanelli and A. Rubbia, Neutrino factories: Detector concepts, presented at the IX Intl. Workshop on Neutrino Telescopes (Venice, Mar. 6-10, 2001), http://pcicarus7.ethz.ch/Talks_pdfs/venice2001.pdf

[56] M.V. Diwan et al., Proposal to Measure the Efficiency of Electron Charge Sign Determination up to $10 \mathrm{GeV}$ in a Magnetized Liquid Argon Detector ( $\mu L A N N D D$ ), submitted to BNL (April 12, 2002),

http://www.hep.princeton.edu/ñcdonald/nufact/bnl_loi/argonprop.pdf

[57] G. Mulholland, ELAN Detector Vessel Prliminary Study, (May 17, 2002), http://www.hep.princeton.edu/m̃cdonald/nufact/mulholland/ELAN_Proposal.pdf 


\section{Appendix A}

\section{Possible Detector Technologies}

\section{A.1 Low Z Tracking Calorimeter}

Identification of the final state electron in a calorimetric detector requires that the sampling frequency is high, of the order of $1 / 4-1 / 3$ of the radiation length $X_{0}$. Neutrino detectors must serve as a target and as a detector at the same time, hence their mass must be maximized. These two requirements lead to a conclusion that the absorber should be made out of a low $\mathrm{Z}$ material to maximize the mass of the detector while maintaining good sampling frequency. Low $\mathrm{Z}$ absorber will lead to a minimal number of the active detector planes, for a given total mass of a detector, hence it will minimize the cost of the detector.

A required transverse granularity of the detector is related to the local particles density on one hand and to the Moliere radius on the other hand. Hadron and electron showers develop over large volumes in a low density detectors, hence the requirements on the transverse granularity of the detector will be relatively modest.

A direct consequence of the large spatial extend of events is a necessity of a fiducial cut of the order of 1 meter away from the edges of the detector. This, in turns requires that the transverse size of the detector is as large as it is practically possible. As an example, with the 1 meter from edges fiducial cut only $81 \%$ of a total mass of a $20 \times 20 \mathrm{~m}^{2}$ detector is in the fiducial volume, for the $12 \times 12 \mathrm{~m}^{2}$ detector it is only $69 \%$. 


\section{A.1.1 Low energy neutrino interactions in low $\mathrm{Z}$ calorime- ter}

Neutrino interactions in the region of $1-3 \mathrm{GeV}$ involve very low multiplicity reactions: quasi-elastic scattering, resonance production, single pion production. Deep inelastic scattering sets on above $2 \mathrm{GeV}$ although multiplicities of the hadronic final states are still very low. $\nu_{e}$ charged current events are recognizable by the presence of an electron in the final state. The electron, on the other hand, will be characterized by the presence of additional hits, due to the onset of the electromagnetic shower, along its trajectory.

In such a low multiplicity environment a low $\mathrm{Z}$ tracking calorimeter will provide a valuable information on the topological properties of the event. This potential is illustrated in Fig. A.1 showing several examples of low energy $\nu_{e} \mathrm{CC}$ events are registered in a plastic calorimeter with $1 / 3 X_{0}$ sampling and with the transverse granularity of $3 \mathrm{~cm}$. Black circles denote the hit detector strips. Colored lines are indicating the initial directions of final state particles: red - electron, blue proton, green - charged pion, yellow - neutron and cyan - gamma.

Typical NC events are not very spectacular - they have just few hits from low energy final state hadrons. More important are the high $y$ events as they constitute potential background for the $\nu_{e}$ sample. Some example of such events are shown in Fig. A.2. Most of them do not represent a serious source of background, it is only the small fraction with relatively energetic $\pi^{0}$ which are problematic. To illustrate the qualitative difference between neutrino interactions at very low energies, in $2 \mathrm{GeV}$ region and at higher energies we show examples of $\nu_{e} \mathrm{CC}$ event and a typical $\mathrm{NC}$ event for neutrino energies of the order of $5 \mathrm{GeV}$ in Fig. A.3.

\section{A.1.2 Electron identification}

Charged current $\nu_{e}$ interactions are identified by the presence of an electron among the final state particles. There are several characteristic features of these events which can be used to differentiate them from the $\mathrm{NC}$ and $\nu_{\mu} \mathrm{CC}$ events:

- they do contain a long track

- the track is accompanied by additional hits due to the onset of an electromagnetic shower

- track energy is a significant fraction of the total energy of the event 

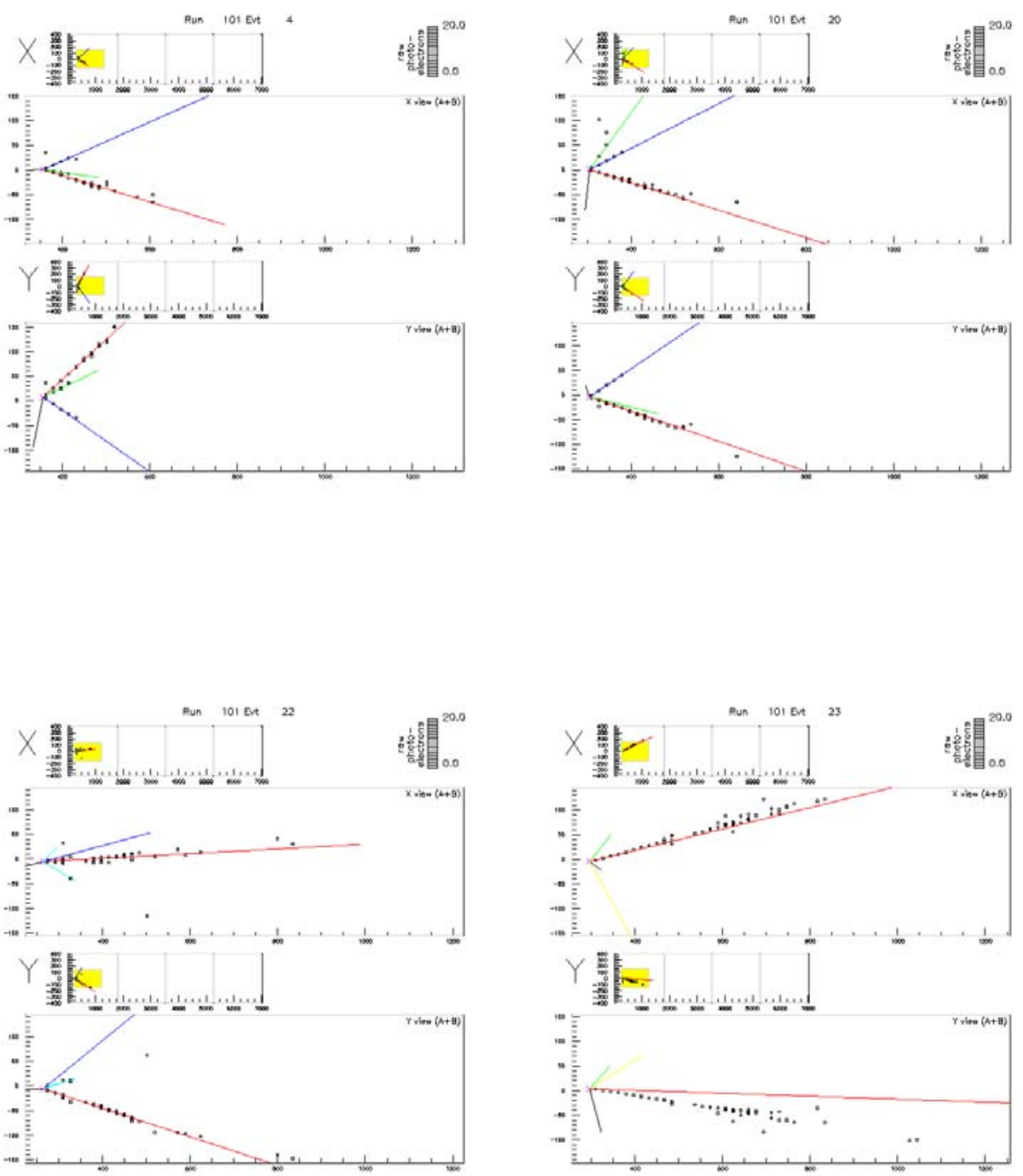

Figure A.1: Typical charged current $\nu_{e}$ interactions with energies $1-3 \mathrm{GeV}$ as detected in a fine grained calorimeter with the longitudinal sampling of $1 / 3 X_{0}$ and the transverse sampling of $3 \mathrm{~cm}$. 

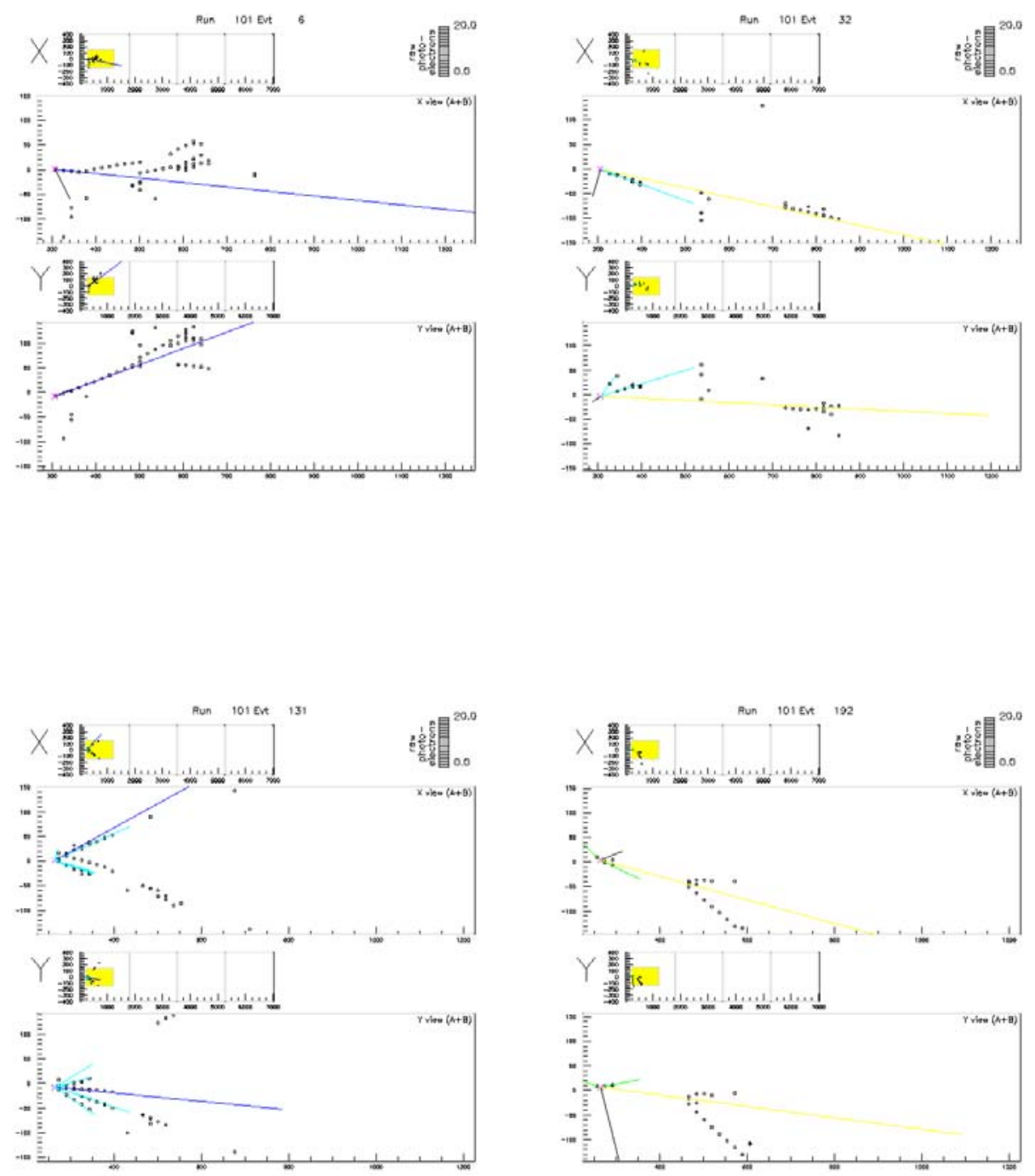

Figure A.2: Typical neutral current interactions with energies $1-3 \mathrm{GeV}$ as detected in a fine grained calorimeter with the longitudinal sampling of $1 / 3 X_{0}$ and the transverse sampling of $3 \mathrm{~cm}$. 

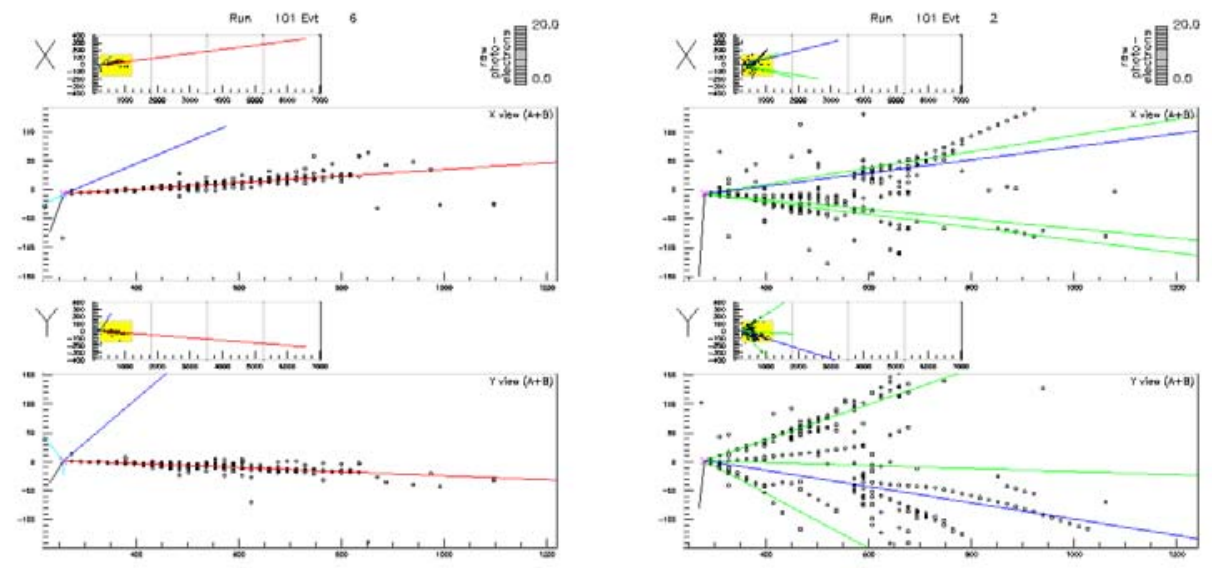

Figure A.3: Typical charged current and neutral current $\nu_{e}$ interactions with energies $\sim 5 \mathrm{GeV}$ as detected in a fine grained calorimeter with the longitudinal sampling of $1 / 3 X_{0}$ and the transverse sampling of $3 \mathrm{~cm}$.

- track direction is at a small or moderate angle to the neutrino beam direction

- the energy deposition at the very beginning of the track is consistent with a minimum ionizing particle

Several independent studies involving full GEANT simulation, realistic reconstruction and analysis of events in the fine grained calorimeter indicate that such a detector enables identification of the $\nu_{e} \mathrm{CC}$ interactions with a typical efficiency $\epsilon=0.35-0.4$ while keeping the contribution of NC contamination at the level of the intrinsic $\nu_{e}$ component of the beam. An example of the analysis chain is shown in Fig. A.4. These results serve as a basis of the estimates of the physics potential of the off-axis experiment presented in this letter. 


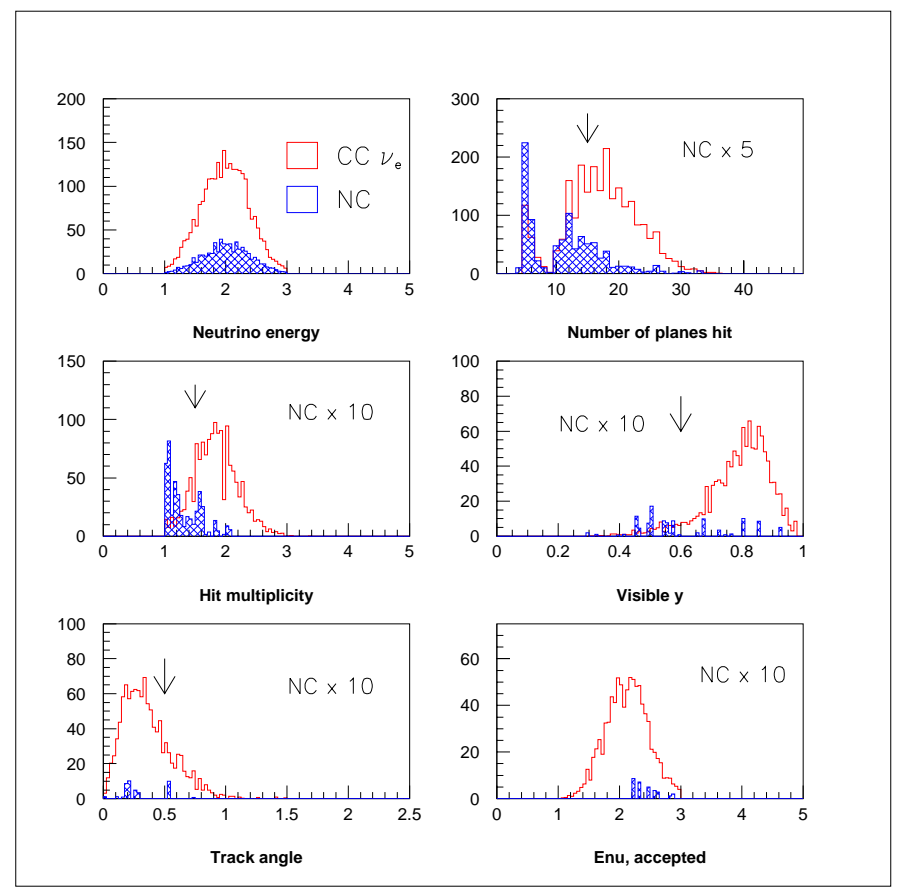

Figure A.4: Typical evolution of the events sample: open histogram for $\nu_{e} \mathrm{CC}$ events, shaded histogram is for the NC sample. From top left to bottom right: Neutrino energy, number of planes in the longest track of the event, average number of hits (per plane) in a road around the longest track, fraction of the event energy contained in the longest track, track angle with respect to the beam direction, neutrino energy of the events passing the cuts. Cut values are indicated by arrows.

\section{A.1.3 Energy resolution}

Energy resolution of the detector is an important parameter. It provides a significant rejection against the backgrounds, as the oscillation signal events are concentrated in a narrow range determined by the beam spectrum, whereas the backgrounds are characterized by a broad distribution of the observed energy.

Good sampling frequency ensures that the sampling fluctuations will be small, hence the energy resolution will be good. This is true even if the readout is purely digital, i.e. the energy is determined by a number of the hit detectors strips, irrespective of the deposited energy per strip.

The resulting energy resolution, $\Delta E / E \sim 16 \%$ is well matched to the off-axis 


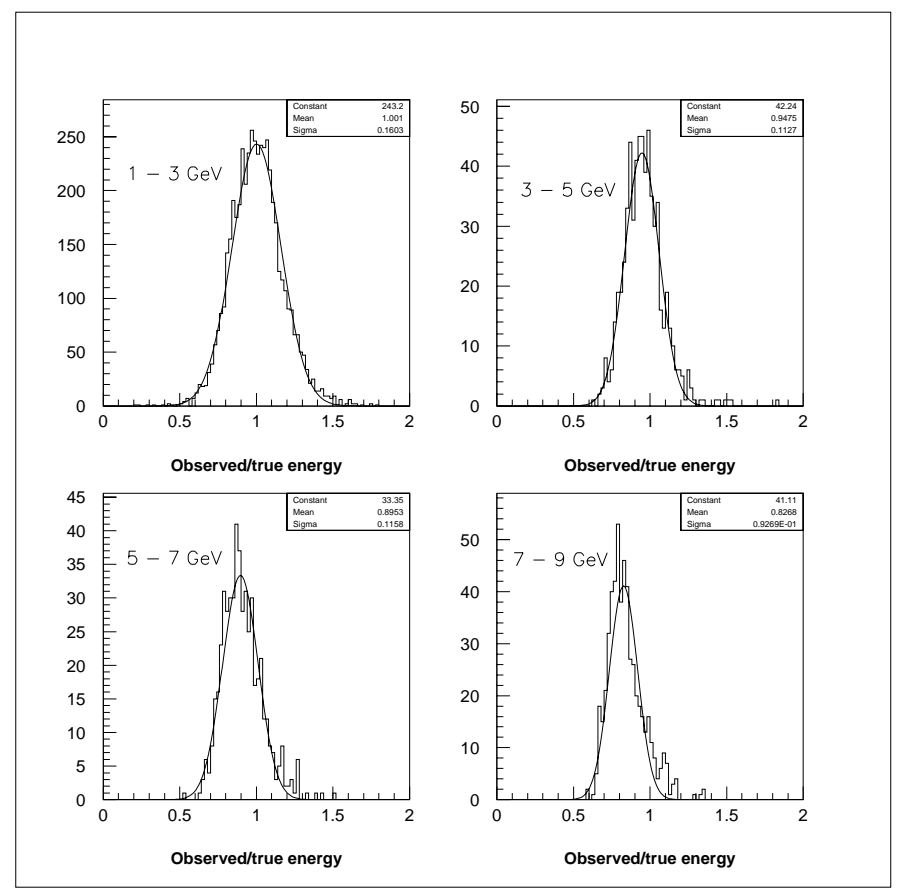

Figure A.5: Total neutrino energy resolution for low y $(y<0.5) \nu_{e}$ CC interactions for different neutrino energies

energy spread. Purely digital readout leads to a non-linearity of the response of the detector, reaching some $18 \%$ at $E_{\nu}=8 \mathrm{GeV}$. This feature, although irrelevant for the neutrino oscillations experiment, can be avoided by having an analog readout, for example in the liquid scintillator design.

\section{A.1.4 A specific detector example: LoDen - LOw DENsity calorimeter}

Neutrino experiments do not present significant challenges to the active detectors. The low cost and long term stability of operations are among the most important characteristics.

The principal challenge of the $\nu_{e}$ detector will be therefore in the engineering area: how to construct as large as possible and as cheap as possible low Z calorimeter. 


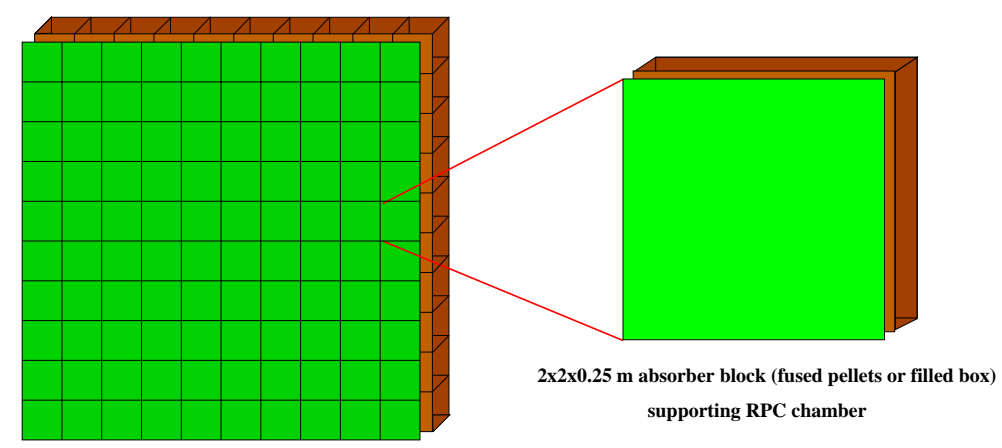

Figure A.6: Modular detector design: $20 \times 20 \mathrm{~m}^{2}$ planes constructed by stacking absorber modules, active detectors supported by the absorber.

The Loden detector consists of the alternating absorber planes, $1 / 3 X_{0}$ in thickness and Glass Resistive Plate Chambers. One detector plane represents a mass of about 70 tons hence 300 planes are necessary to attain the mass of $20 \mathrm{kton}$. Total area of the active detectors is $120,000 \mathrm{~m}^{2}$.

The choice of the Glass RPC as a an active detector is primarily dictated by the low unit cost of such detectors. These detectors have several other attractive features: they are robust and reliable. They allow physical decoupling of the active elements (spark gaps) from the readout elements (pick-up strips) making it relatively easy to construct a very large area detector with relatively few readout channels out of a large number of smaller, hence manageable, individual detectors. Glass RPC are read out via pick-up strips providing $x$ and $y$ coordinate in every plane. Optimal transverse granularity of the active detectors needs to be studies in details, but the initial investigations indicate that a strip readout with a strip width of $3 \mathrm{~cm}$ is adequate. Such a granularity leads to some $\sim 1400$ readout channels per plane, or 400, 000 channels in total.

Practical design of the detector needs to be validated by the detailed engineering studies. Preliminary calculations indicated that the absorber planes of dimensions approaching $20 \times 20 \mathrm{~m}^{2}$ can be constructed in a self-supporting manner, not requiring external support structures. To prevent potential buckling the absorber planes will be bolted around the periphery and along the center line into modules involving 20 planes.

Practical aspects of constructing very large but relatively thin detector walls must not be underestimated. It would be very desirable to develop a detector design based on a 'human' scale modules, say $2 \times 2 \mathrm{~m}^{2}$ which could be stacked to construct w final large detector plane, as illustrated in Fig. A.6. 


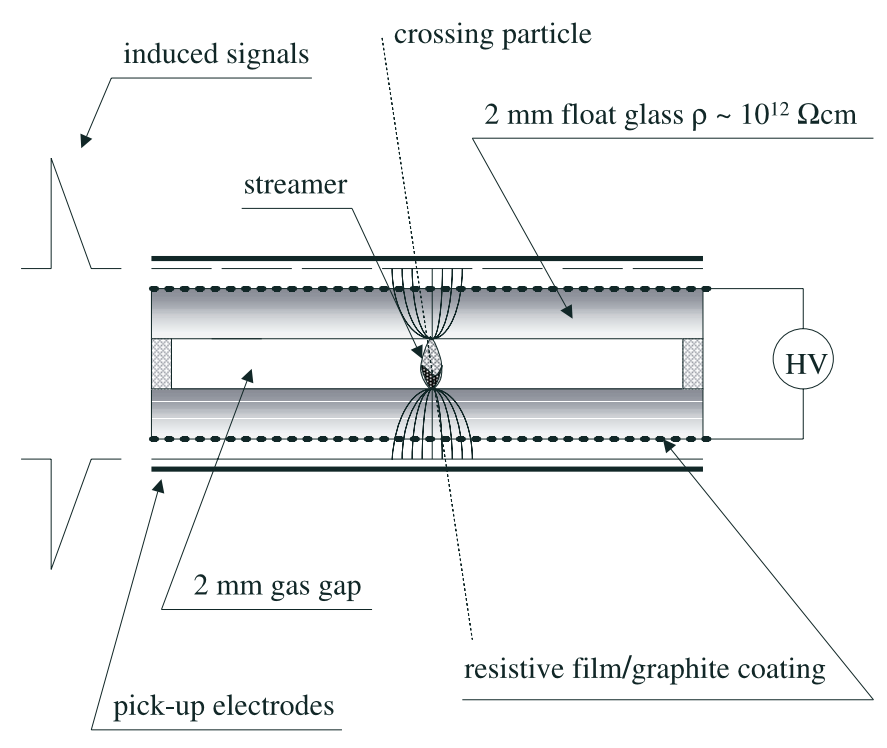

Figure A.7: Glass RPC detector principle

\section{A.1.5 Glass RPC Chambers}

Glass RPC chambers belong to a family of the Resistive Plate Chambers, but they utilize inexpensive commercial float glass of high resistivity $\left(10^{12} \Omega \mathrm{cm}\right)$ instead of the traditional bakelite.

The detector, shown in Fig. A.7, is composed of two parallel glass electrodes, $2 \mathrm{~mm}$ thick, kept $2 \mathrm{~mm}$ apart by appropriate spacers. The gap between electrodes is filled with a suitable non-flammable gas mixture. The resistive coating on the outer surfaces of the glass connected to the HV power supply creates a strong electric field $\sim 4 \div 5 \mathrm{kV} / \mathrm{mm}$ across the gap. An ionizing particle initiates a local discharge which induces a signal on external pickup strips. The induced pulses are typically $100-300 \mathrm{mV} / 50 \Omega$ with $\sim n s$ time resolution. Large signals of $100-200 p C$ allow for the possibility of a variety of cost saving options in the readout electronics.

This type of detector has been used successfully for muon and K-long detection in the BELLE experiment at KEK [33]. A single detector plane has an efficiency of typically $90-95 \%$. Double planes in the BELLE experiment were used to provide $99 \%$ efficiency. For this experiment we expect single planes to be adequate. 
The high resistivity of the glass and the quenching properties of the gas limit the discharge to a small area. This type of detector does not have the rate capability necessary for hadron colliders but, as demonstrated by BELLE, is more than adequate for this neutrino experiment. They are ideal for large area applications since the pickup strips can be configured as transmission lines covering many meters in length.

These detectors are relatively simple and inexpensive to construct. The typical cost of the detectors is in the range of $\$ 100-150 / \mathrm{m}^{2}$ of the detector [34, 35]. They have been shown to be stable and reliable in more than four years of operation at KEK.

These low cost detectors which can be configured to cover a large area. As an example, the Monolith [36] group has developed a design for the RPC planes as large as $15 \times 30 \mathrm{~m}^{2}$. This group also developed several possible industrial production techniques whicch may allow further cost savings. 


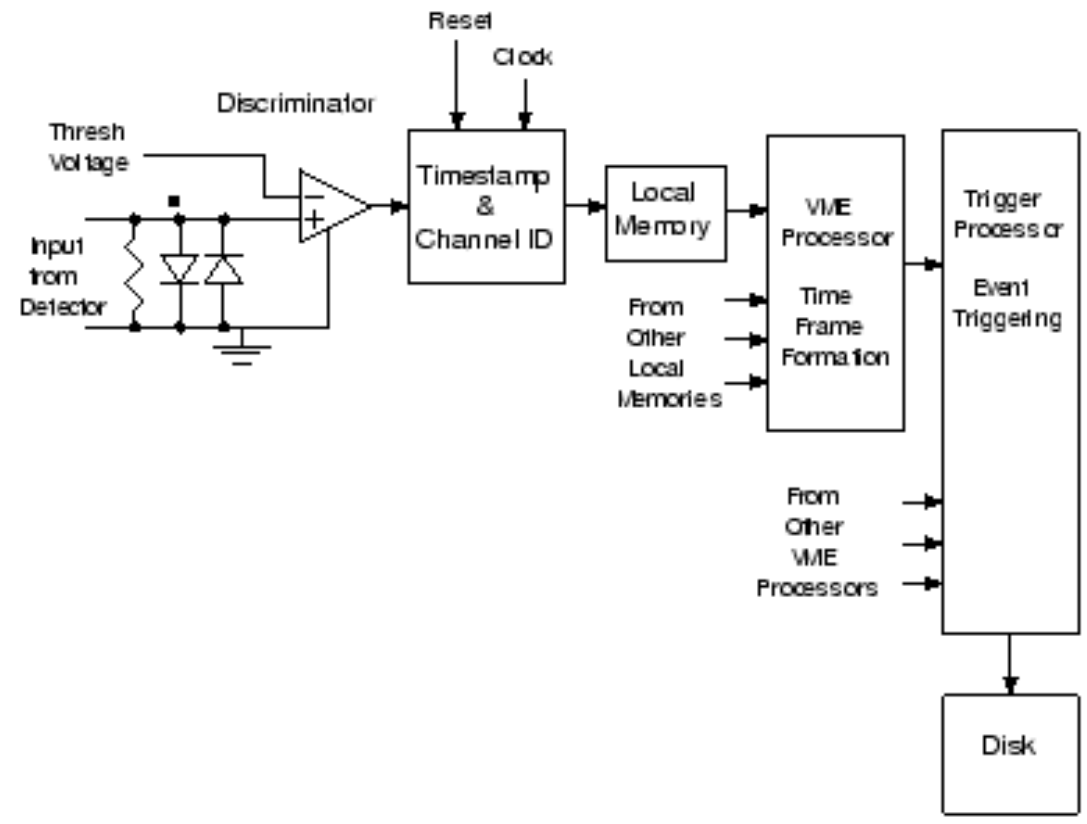

Figure A.8: Block diagram of the readout electronics

\section{A.1.6 Readout Electronics}

When operated in streamer mode, RPC detectors produce a large pulse in response to the ionization within the gas. The signals are large so that a significant voltage (100 mV or more) can be developed across a 50 or $100 \mathrm{ohm}$ resistor. Because the measurement of events requires only the observance of hits in the detector, it is sufficient to use a simple discriminator as the front-end electronics, without the need for additional amplification or signal processing.

To facilitate event reconstruction, the output of each discriminator would latch a time-stamp for the event. The time-stamp is formed using local counters, which receive a common clock and counter reset signal from a global timing system. In this way, all time-stamp counters across the detector are synchronized. When an RPC channel is hit, the data that is recorded is the value of the time-stamp counter. The time-stamp hits are stored in a local memory, and read out later. A block diagram is shown in Fig. A.8. The resolution of the time-stamp is determined by the clock speed, which might be $100 \mathrm{~ns}$ (10 MHz.) The number of bits in the counter is determined by the frequency of the counter reset, which might be $0.5 \mathrm{~Hz}$, corresponding to frequency of the NuMI beam spill. 
RPC ASIC

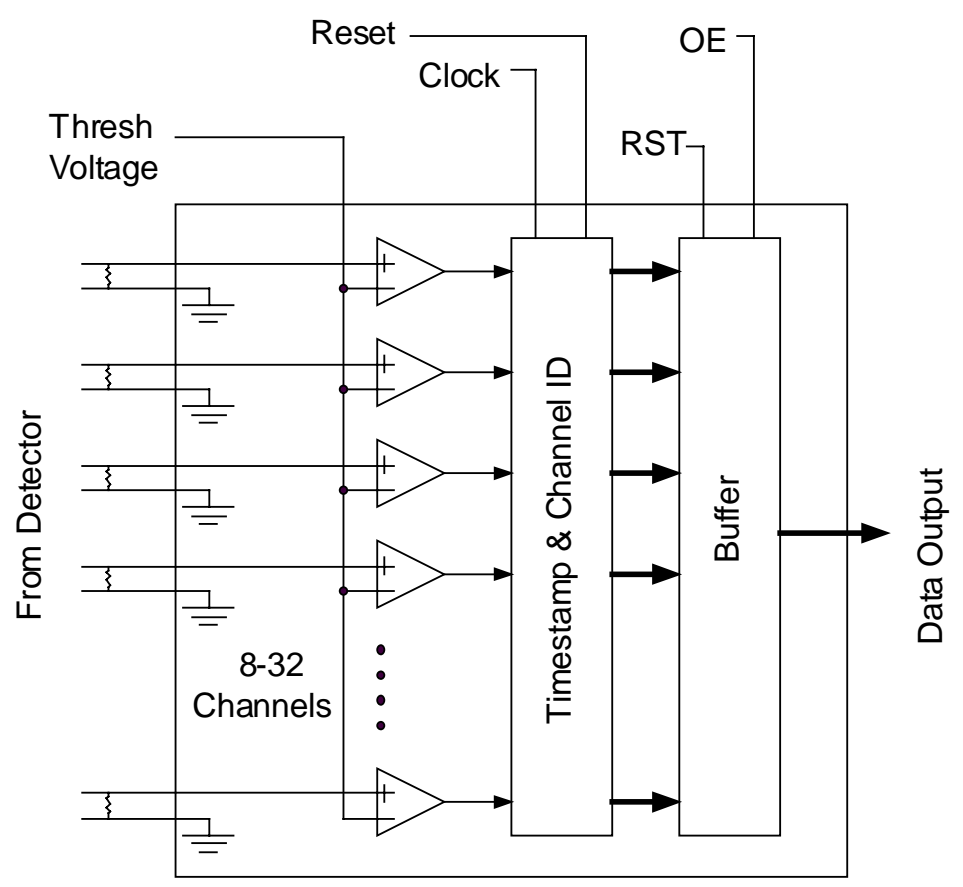

Figure A.9: ASIC for the front-end readout electronics

Because the data rate is low, it is envisaged that no trigger hardware is needed. Instead, the formation of a trigger and the analysis of events are done using a series of processors. This is similar to the data acquisition system of MINOS[11]. The initial sorting of hits by time-stamps is done using a VME-based processor in the front-end crate. The processor would form "time frames" using the timesorted data. The time frames are then sent to a trigger processor, which receives time frames from the entire detector. The trigger processor runs algorithms that look for tracks and discard noise hits. Those events that pass are either written to disk, or passed to another processor for further analysis.

A goal in the electronics development is to reduce the cost. Given the high channel count and relatively simple front-end configuration, it is practical to implement the functionality in a custom ASIC. The comparator and time-stamp functions are easily realized in silicon. The cost per channel for the ASIC would be $\$ 1.50$ for a 16 -channel device. A block diagram of the chip is shown in Fig. A.9.

The front end electronics would reside in racks situated close to the detector, as 


\section{Physical Configuration of Electronics}

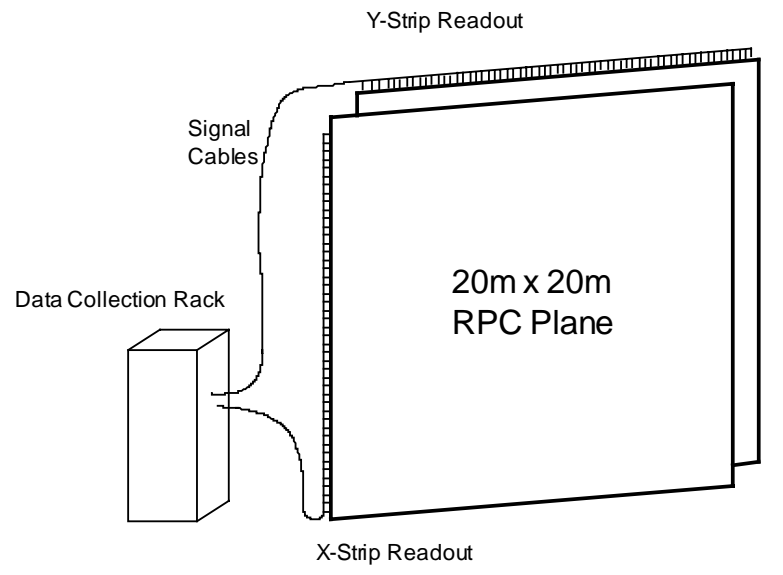

Figure A.10: Physical layout of the readout electronics

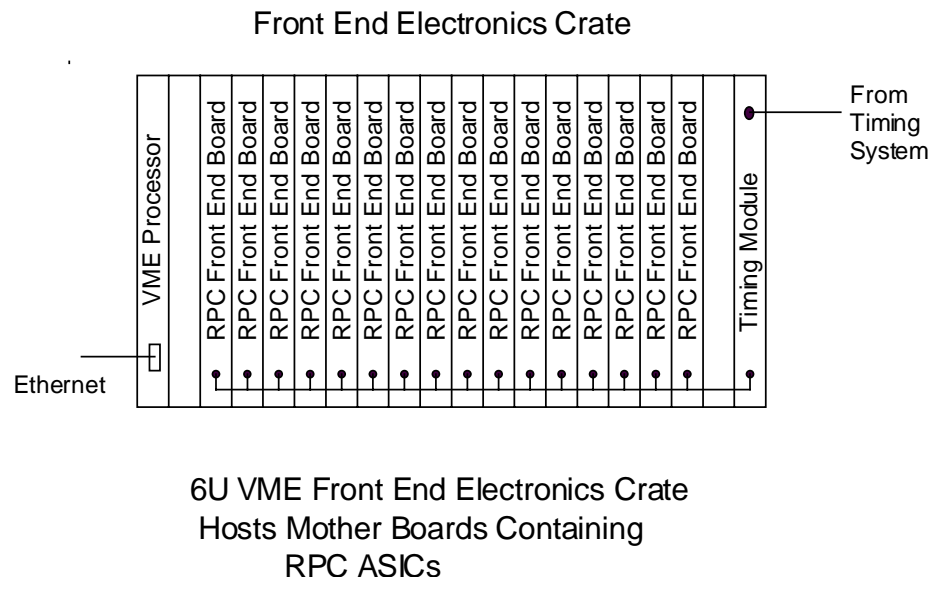

Figure A.11: Front end electronics crate

shown in Fig. A.10. The ASICs would be mounted on VME boards, providing easy access of the data to the front-end processor. Each front-end board would host 16 chips, servicing 256 detector channels. A VME crate would hold 16 such cards, servicing 4096 channels, as shown in Fig. A.11.

A total of 100 front crates would be needed for $400 \mathrm{~K}$ channels in the detector. 


\begin{tabular}{|c|c|c|c|}
\hline ITEM & $\begin{array}{l}\text { Unit } \\
\text { Cost }\end{array}$ & $\begin{array}{l}\text { Number } \\
\text { Needed }\end{array}$ & Subotal \\
\hline 16-Ch ASICs & $\$ 25$ & 25,000 & $\$ 625 \mathrm{~K}$ \\
\hline Front End Boards & $\$ 700$ & 1,600 & $\$ 1200 \mathrm{~K}$ \\
\hline $\begin{array}{l}\text { VME Crates, Power } \\
\& \text { Processors }\end{array}$ & $r, \$ 5,000$ & 100 & $\$ 500 \mathrm{~K}$ \\
\hline Trigger Processor & $\$ 20,000$ & 1 & $\$ 20 \mathrm{~K}$ \\
\hline Cables, Misc & $\$ 100$ & 1,600 & $\$ 160 \mathrm{~K}$ \\
\hline Total & $\$ 6.25$ & 400,000 & $\$ 2505 \mathrm{~K}$ \\
\hline
\end{tabular}

Figure A.12: Cost estimate of the front-end electronics

A preliminary cost estimate is shown in Fig. A.12. The total cost of the electronics is estimated to be $\$ 2.5 M$, or $\$ 6 /$ channel. 


\section{A.1.7 Ancillary Systems}

Assuming that the RPC mechanics and electronic readout are engineered to minimize the cost of their fabrication, it will be important to take care in the design of the ancillary systems, namely the gas mixing and distribution system and the HV system.

\section{A.1.7.1 Gas System}

The design of the gas system must address a number of challenges:

- the number of distribution channels will be large;

- the gas entering the chambers must be very dry;

- safety;

- cost of operation.

Although it may be possible to feed the gas in series through multiple gas volumes, there will be limits on this since the accumulated effect of the flow resistance of the chambers will eventually result in a pressure that exceeds the limit of the glue joints (the geometry of the RPCs is by its nature very weak with respect to internal overpressure). Moreover, experience with series connection of the Belle RPCs showed that the chambers near the end of the line were slow to achieve stable operation. Thus it will be important to minimize the perchannel cost of the distribution system and to develop a design that provides a balanced flow between channels without the need for extensive adjustment. To solve this problem, Belle used "flow resistors," which are $10 \mathrm{~cm}$ stainlesssteel tubes drilled down the center with sub-mm bores. These tubes are series inserted in the output of each channel and set the flow impedance of the channel. This provides a simple and economical way to balance the flow from channel to channel without need for tedious adjustment.

Keeping the gas dry (water vapor at the 100 ppm level or less) is very important to avoid formation of hydrofloric acid, which etches the glass, ultimately destroying the RPCs (Belle came very close to having this happen). Achieving the requisite dryness is reasonably simple provided copper tubing is used (plastic tubing is too permeable).

The importance of safety and economy are clear and will no doubt be the subject of extensive design studies. 


\section{A.1.7.2 HV}

Perhaps the most challenging aspect of the RPC high voltage system is the voltage across the gap must be in the neighborhood of $8 \mathrm{kV}$. Although it is possible to achieve this with a single ended supply, supplies capable of sourcing more than $5 \mathrm{kV}$ tend to be expensive on a per-channel basis. For that reason the Belle experimented employed a differential scheme, wherein the total bias of $8 \mathrm{kV}$ was achieved by using a pair of supplies operating at opposite polarities. A single $0-6 \mathrm{kV}$ supply was used on the positive side. Since these units were somewhat pricey, several RPCS were ganged onto a single supply. For the negative bias, independent (actually only quasi-independent) supplies were used on each channel. This allowed Belle to monitor the current on individual RPCs, which proved to be very important in the operation of the system, as discussed below.

Experience with previous RPC HV distribution systems indicates that it is essential to monitor the current drawn by the RPCs on a per channel basis (the dark current of an RPC is an excellent diagnostic of its overall health). The ability to switch a single chamber on or off is also useful, although perhaps not essential (one could always resort to simply unplugging the cables of misbehaving chambers). Still less important is the ability to adjust the $\mathrm{HV}$ on individual chambers.

Once again safety and cost will be important practical considerations. 


\section{A.1.8 Modular Detector Design}

One possibility for the detector assembly is to use pre-engineered intermodal containers as the basic modular unit and then replicate the unit many times to create a giant structure. Dry Freight containers are used for international exchange of over $90 \%$ of the world's manufactured goods and can be transported easily by truck, rail, and sea (therefore termed "intermodal"). These objects are in widespread use throughout the world and the existing mass production infrastructure offers a low cost solution for a low-density neutrino detector.

The basic intermodal container is a steel box $8 \mathrm{ft}$ wide by $8 \mathrm{ft} 6$ inches high by $20 \mathrm{ft}$ long with an empty weight of about $2,300 \mathrm{~kg}$ and a payload capacity of $22,000-28,000 \mathrm{~kg}$ depending on the manufacturer. These containers have internal volumes of $31-36 \mathrm{~m}^{3}$ and therefore can hold cargo with an average density of about $0.7-0.8 \mathrm{~g} / \mathrm{cc}$. The box has rails around the edges, a set of steel floor supports, a pair of doors at one end, and is typically covered with corrugated steel. Such a container is called a "Twenty-foot Equivalent Unit" or TEU. The International Standards Organization specification calls for the TEU box to be based around 4 corner posts that can each take a load of $86,400 \mathrm{~kg}$. This ISO specification means that the boxes can be stacked 9 high when fully loaded and they are routinely stacked this high in large ocean-going container ships. The cost of a TEU (in quantity) is in the range $\$ 1500-\$ 2500$. Millions of TEUs exist and hundreds of thousands of new ones are built each year.

A modular neutrino detector, $18 \mathrm{~m}$ by $20 \mathrm{~m}$ by $80 \mathrm{~m}$, could be assembled from about 800 such TEUs at a cost of approximately $\$ 1.6 M$. The resulting structure would be self-supporting and weather-tight. The empty containers by themselves would constitute about 1600 metric tons of steel. Off-the-shelf pre-engineered gantry cranes, lifting fixtures and many other attachments like box-to-box clamps and casters are available.

The small size of the basic TEU would allow construction of detector modules at many institutions around the country. The intermodal feature would allow easy shipment between institutions for different steps in the assembly process and the completed TEUs could easily be shipped to any site along the NuMI beamline. The modular structure allows for mobility of the detector if a different off-axis position becomes desirable part way through data collection, or if a different neutrino beam eventually becomes available from a superbeam or from a muon storage ring.

The concept does have two drawbacks. First, temperature control is not a built in feature of containers, but this can likely be solved using excess load capacity of the stacked TEUs as the supporting structure for an insulating shell. Secondly, when the TEU boxes are stacked, the floor supports carrying the load in each 
individual TEU mean that the assembled detector will have an un-instrumented area with an active detector to active detector vertical crack size of about 20 $\mathrm{cm}$. Both of these problems require further study. 


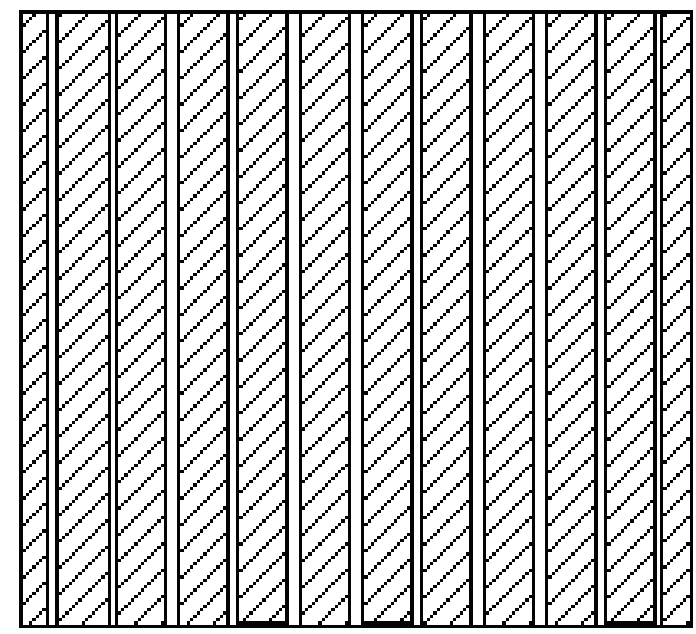

Figure A.13: Container $2.1 \times 2.3 \mathrm{~m}^{2}$ cross section filled with target slabs creating 10 active gaps, $5.4 \mathrm{~cm}$ wide.

\section{A.1.9 Absorber Construction and Composition}

Typical low Z absorber materials have a density of $0.7 \mathrm{~g} / \mathrm{cm}^{3}$, and a radiation length of about $64 \mathrm{~cm}$ (similar to a mixture of $70 \%$ polyethylene and $30 \%$ air) leading to absorber elements $16-20 \mathrm{~cm}$ thick.

Modular construction techniques include: standardized shipping containers filled with alternating layers of target and active detector, layers of target and active detector supported by an external framework, and free-standing structures of the target material with regularly spaced gaps for active detectors.

\section{A.1.9.1 Containers and Loose Fill Materials}

Standard shipping containers are inexpensive and strong enough to be stacked at least 9 containers high. The effects of unsampled target and voids in a container array are unlikely to cause serious problems but must be investigated. Using aluminum (instead of steel) containers may reduce the unsampled energy loss, and the inactive volume can used for service and signal connections between containers. The interior of a standard shipping container has the dimensions $(h \times w \times l) 2.1 \times 2.3 \times 5.8 \mathrm{~m}^{3}$. The interior $230 \mathrm{~cm}$ width can be divided, as shown in Fig. A.13, into 12 layers of target material $(10 \times 16 \mathrm{~cm}+2 \times 8 \mathrm{~cm})$ with 


\begin{tabular}{|c|c|c|}
\hline Loose Fill Material & Density $\left(\mathrm{g} / \mathrm{cm}^{3}\right)$ & Price $(\$ / \mathrm{lbs})$. \\
\hline Plastics (recycled) & $0.6-1.0$ & $0.10-0.30$ \\
\hline Shredded Tires & $0.4-0.8$ & 0.15 \\
\hline Walnut Shells & $0.6-0.7$ & 0.1 \\
\hline Cracked Corn & 0.7 & 0.05 \\
\hline
\end{tabular}

Table A.1: Typical Low Z Fill Materials

$10 \times 5.4 \mathrm{~cm}$ slots for active detectors. Assuming a target density of $0.7 \mathrm{~g} / \mathrm{cm}^{3}$, the container holds $15450 \mathrm{~kg}$ of target mass, well below the load limit.

Target layers can use a loose fill if contained in a cavity formed by (wood) walls. To prevent bulging, however, the cavity might require an internal bracing. Low $\mathrm{Z}$, loose fill materials include recycled petroleum based or agricultural products. Some typical loose fill materials are listed in Table A.1.9.1.

Recycled fill materials include plastics that are clean but can obtained only in relatively small batches with large variations in properties, and shredded tires which are quite stable and can be had in various particle sizes. The least expensive loose fill materials are agricultural products that include nutshells and cracked (and dried) corn. However, a polypropylene bag might be required to package these materials. Better yet, loose fill material can be molded into precast blocks, but add significantly to the cost.

\section{A.1.9.2 Molded Particleboard}

A standard building material called particleboard, e.g., Georgia Pacific's (GP) MicroFine Novoply, is likely the cheapest (about $\$ 0.123 /$ lbs.) commercially available low-Z molded product. Particle board has very good mechanical properties and can produced in $8^{\prime} \times 28^{\prime}(8.3 \mathrm{~m})$ sheets up to 1.44 " thick. The boards can be laminated into slabs of the appropriate thickness, and installed in the containers, as shown in Fig. A.13. This would form smooth and straight gaps for the active elements.

Particle board for a $20 \mathrm{kT}$ detector would cost $5.5 \mathrm{M} \$$.

\section{A.1.9.3 External Framework and Self Supporting Structures}

Particleboard, laminated into target slabs, 8 or $16 \mathrm{~cm}$ thick, can be bolted together and then supported by an external framework to generate a large array 
of target and active elements. However, particleboard might be strong enough to create a stable (container sized) module $\left(2.1 \times 2.3 \times 5.8 \mathrm{~m}^{3}\right)$ with, as described earlier, gaps for the active elements. Bonding or bolting a steel skin to four sides (top, bottom, and both ends) would stabilize the module against flexing. For active elements that must run the full height of the target, e.g., liquid scintillator filled tubes, internal bracing between the target slabs (instead of an external steel skin) can create continuous vertical slots. 


\section{A.1.10 Liquid Scintillator Option}

Liquid scintillator makes an ideal active detector for a large inexpensive tracking calorimeter. It is a proven technology that has been used in large quantities in detectors over long periods of time. It gives good energy resolution and charged particle tracking efficiency. Liquid scintillator allows for a flexible geometry and segmentation that can be optimized for the expected events. Because it is a liquid, it can be added to the detector after it is assembled to minimize assembly cost. It can even be removed if repair is necessary or if the detector needs to be disassembled and moved. Modern off-the-shelf photonics can be used to readout the detector so that additional electronics is minimal. Scintillator segmented into cells gives both pulse height information and tracking. Since this type of detector was investigated as a candidate for MINOS, very little additional R\&D and engineering is required to construct a $20 \mathrm{kTon}$ detector from this technology.

\section{A.1.10.1 Support Structure}

As a specific example, we present a conceptual design of a detector based on liquid scintillator interspersed between planes of water approximately $1 / 3$ of a radiation length thick. Both the liquid scintillator and the water would be contained in modules of extruded PVC plastic colored by titanium dioxide for good reflection. Although this is a conceptual design, its structure has been investigated previously and those investigations documented [37].

The design we consider here is based on modules that contain 6 layers of 30 cells each with the dimensions of each cell $3.3 \mathrm{~cm} \times 3.3 \mathrm{~cm} \times 12 \mathrm{~m}$ long. The outer walls are $1 \mathrm{~mm}$ thick while the inner webbing of the extrusion is $0.5 \mathrm{~mm}$ thick. One of the layers would hold liquid scintillator and the others would hold water. Although the cell sizes are chosen here for ease of calculation and would be optimized by Monte Carlo studies of neutrino events together with structural studies of the plastic, they are close to those required for a final detector and are structurally sound.

For each module, the cells are sealed at one end by a single plug of PVC glued in place. This construction has been tested to be reliable with no leaks to a pressure of over 6.5 atmospheres. If the detector consists of scintillator planes separated by $1 / 3$ radiation length of water as the target mass, a $12 \mathrm{~m} \times 12 \mathrm{~m}$ x $300 \mathrm{~m}$ detector will have a $20 \mathrm{kT}$ fiducial mass (assuming a $1 \mathrm{~m}$ fiducial cut around the edge.) Alternate planes of scintillator/water modules would have perpendicular cells to give an $\mathrm{X}-\mathrm{Y}$ readout. This geometry would give 1500 planes of liquid scintillator. 


\section{A.1.10.2 Signal Collection}

Light would be collected using a $1 \mathrm{~mm}$ diameter wavelength shifting fiber similar to that used in MINOS. Each cell would have a $12.5 \mathrm{~m}$ long fiber placed in it. Our previous studies have shown that the amount of light collected by the fiber is not sensitive to the position of the fiber in the tube. The end of the fiber will dipped in white paint and then in epoxy to provide $30 \%$ reflectivity at the end. Tests have shown a minimum ionizing particle this geometry with BC517L scintillator will give 40 photons from the end of the fiber. The fibers from module would be gathered in a manifold to an optical connector similar in design to that of MINOS. The design of the PVC manifold would also include fittings to fill and, if necessary empty the cells of scintillator. Chemical activity tests show [37] there will be no measurable effect of the scintillator on either the fiber or the PVC extrusion over the lifetime of the experiment.

\section{A.1.10.3 Support}

The construction of the detector is simplified because the light plastic extrusions would be stacked to form the detector before the liquid was added. Mounting the plastic extrusions with alternating planes at $45^{\circ}$ to the horizontal and $45^{\circ}$ to the vertical would allow the detector to be read out and filled from the top. A possible mounting structure would be a $\mathrm{V}$ shaped trench. The sloping walls could be supported by earth in a manner similar to culvert wall construction. The longitudinal direction the trench would be sloped at about $1^{\circ}$ for ease of construction and to allow drainage for water seepage from the ground.

The stacked extrusions would be supported primarily by the sides of the trench and at the ends of the trench by a bookend structure. Most of the weight of the detector is supported by compression on the floor of the trench carried by the entire length of the extrusion. In this configuration, all mechanical stresses are well below the tested strength of the extruded structure. We envision that the detector would be covered with a roof, possibly of Quonset hut design, which would then be covered by about 3 meters of earth. This would ensure a stable operating temperature for the detector and eliminate the soft component of the cosmic ray flux.

\section{A.1.10.4 Readout}

Image intensifiers provide a low cost readout well matched to the rates of cosmic rays through the detector. Reading out fibers into a standard $25 \mathrm{~mm}$ image intensifier will require one image intensifier for every plane of the detector. 
Standard image intensifiers have a quantum efficiency of better than $10 \%$ in the green and a gain of at least $10^{5}$. An image intensifier would be read out by a video camera. The CCD in a modern off-the-shelf video camera can be gated to have an exposure of as little as 10 microseconds and can be read out at 30 $\mathrm{Hz}$.

For this experiment we propose an exposure gate of 20 microseconds. Even on the surface, the occupancy rate of a single cell would then be about $1 \%$. The 360 cells of each plane of the detector could be read out by a single image intensifier and video camera. Standard firewire readout would be done into processor for each camera, the processors would be sparsified and read into a PC. If necessary, several PCs would alternate spills to assure adequate readout time.

\section{A.1.10.5 Cost Estimate}

The costs of constructing the detector described above is based on quotes and engineering estimates of the liquid scintillation detector proposed for MINOS[37]. It is expected that the cost of the 20 kton detector, excluding support structures and building, is of the order of $\$ 40 M$. 


\section{A.1.11 Backgrounds in a surface detector}

\section{A.1.11.1 Cosmic ray rates in the detector}

At the surface of the earth, the products of the extensive air showers initiated by primary cosmic rays high in the atmosphere provide a background flux to any detector. We present some preliminary estimates of these rates.

The principle component is muons with a flux of $110 \cos ^{2} \theta / m^{2} / s r / s[38]$. The net flux on a detector of area $3000 \mathrm{~m}^{2}$ is then about $7 \times 10^{5}$ muons per second. The average energy of these muons is $4 \mathrm{GeV}$ and about $30 \%$ will stop inside a detector of average thickness $1200 \mathrm{~g} / \mathrm{cm}^{2}$.

The live-time of the off-axis detector is given by the product of the $20 \mu \mathrm{s}$ spill length and the $10^{7}$ spills per year $=200$ seconds. There are about $1.4 \times 10^{8}$ muons passing through the detector in this time. In any given $20 \mu \mathrm{s}$ spill there are an average of 14 muons distributed throughout the detector.

Accompanying these muons are low energy electrons and photons with net flux about $30 \%$ of the muons and with energies in the range $\sim$ tens of $\mathrm{MeV}$. In addition, there is a small component of low energy neutrons and protons (the proton flux is $\sim 0.1$ the neutron flux) with net vertical flux approximately $3 \times E^{-1.5} / \mathrm{m}^{2} / \mathrm{sr} / \mathrm{s}(\mathrm{E}$ in $\mathrm{GeV})$ [39], i.e. the flux of neutrons with energy above $2 \mathrm{GeV}$ (the onset of inelastic pion production) is about 1.0\% of the muon flux. A small overburden is sufficient to effectively remove the soft electromagnetic component but it requires about $3 \mathrm{~m}$ of rock or earth ( $\sim 10$ interaction lengths) to attenuate the neutron flux so that there will be $\sim 50$ inelastic neutron interactions per year. Pattern recognition plus a moderate fiducial cut will be enough to make this small remnant negligible.

\section{A.1.11.2 Tertiary particle production by muons interacting in the environment outside the detector}

There will be a small flux of electrons and photons produced by muon bremsstrahlung in the overburden directly above the detector. This is a very characteristic signal that is trivially accounted for by a small fiducial cut. A neutral hadronic component of such interactions is potentially more serious.

An estimate of the rate of hadronic interactions of the muons passing through the detector can be made by finding the equivalent spectrum of photons using the Weizsacker-Williams formalism coupled with cross-sections for real photons. 
This has been done by Perkins with the result $d N / d E \approx(0.61 / E) \times 10^{-6} / \mathrm{g} / \mathrm{cm}^{2}$ where $E$ is in $G e V$ [40]. Integrating this over the muon energy spectrum we find that the probability of any single muon depositing more than $2 \mathrm{GeV}$ hadronic energy in one interaction length $\left(\sim 100 \mathrm{~g} / \mathrm{cm}^{2}\right.$ of water or other low-Z material $)$ is $\sim 5 \times 10^{-5}$. The fraction of these interactions giving a final state neutron with energy above $2 \mathrm{GeV}$ is $\sim 10^{-3}[41]$. Estimating the relevant muon flux as $10^{8}$ year and taking the angular acceptance into account, the corresponding flux of energetic neutrons into the detector is $\sim 1 /$ year.

\section{A.1.11.3 Particle production by unseen muons inside the detector}

The proposed detectors contain planes of inert material. A cosmic ray muon can pass through one of these regions undetected but might produce a hadronic or electromagnetic shower which is detected. The probability that a muon will pass through one of these spaces is approximately $w / L$ where $w$ is the width of the inert space and $L$ the transverse size of the detector. We will assume that this probability is $1 \%$. Any such interaction will have an energy flow exactly orthogonal to the events of interest. Simple pattern recognition should make any bremsstrahlung-induced background negligible.

Based on the previous discussion, the probability of a hadronic interaction ( $>2 \mathrm{GeV}$ ) in the full $12 \mathrm{~m}$ thickness of the detector is $\sim 6 \times 10^{-4}$. Thus the net background comes from a potential $1.4 \times 10^{8} \times .01 \times 6 \times 10^{-4} \approx 1000$ muon hadronic interactions/yr. The highest energy pions in these interactions are produced in the forward direction (vector dominance) which is again exactly orthogonal to the events of interest. Large angle pion production occurs at a rate comparable to the large angle nucleon production and will be accompanied by significant nuclear break-up. Pattern recognition must reduce this background to a negligible level.

\section{A.1.11.4 Gamma rays}

There will be a gamma ray flux around the periphery of the detector coming from decay of ${ }^{40} \mathrm{~K}$, and the $\mathrm{U}$ and $\mathrm{Th}$ decay chains in the rock. These are low energy gammas which will give very small signals in the detectors; the net rate will be similar to that in the MINOS detector, i.e. $1-2 \mathrm{kHz} /$ detector plane. 


\section{A.1.11.5 Measurement of cosmic ray backgrounds}

Very accurate background measurements will be made in a relatively small test setup, as the effective lifetime of the neutrino detector is of the order of $200 \mathrm{~s}$ per year of operation. These measurements will validate the correctness of the above estimates.

During the duration of the experiment the background rates will be monitored by triggering the detector during the beam-off time. 


\section{A.2 Water Cherenkov option}

Large volume water Cherenkov detectors provide a proven technology that must be considered as an off-axis neutrino beam far detector. The Super-Kamiokande detector is already planned as the far detector for the JHF neutrino beam[20]. Whether or not water Cherenkov is appropriate for the NuMI off-axis beam depends critically on the difference between the $0.8 \mathrm{GeV}$ peak neutrino energy planned for JHF and the $2 \mathrm{GeV}$ peak neutrino energy anticipated for the NuMI project. At the low energy of KEK/JHF beams the 22.5 kton Super-Kamiokande has proven capability for separating $\nu_{e} \mathrm{CC}$ events from NC $\pi^{0}$ background due to:

- quasi-elastic kinematic constraints,

- good $\pi^{0}$ reconstruction efficiency due to a larger opening angle between the gamma showers,

- low intrinsic NC $\pi^{0}$ background due to the low neutrino energy and small cross section for heavy resonance channels such as the delta.

These advantages can be recovered in the NuMI project by going further off-axis to utilize the 2nd oscillatory maximum; this will be briefly discussed at the end of this section.

The advantages of water Cherenkov for $2 \mathrm{GeV}$ off-axis experiment are:

- it is a mature and proven technology,

- the cost of such a detector can be accurately estimated,

- the cost per kiloton is low, no higher than $\$ 2 \mathrm{M} /$ kton $^{1}$,

- the energy resolution is good, for example at Super-K one finds for electromagnetic showers that $\sigma / E=0.5 \%+2.5 \% / \sqrt{E}$,

The experimental challenge facing this detector option is accurately counting a small sample of $\mathrm{CC} \nu_{e}$ interactions over a background of $\mathrm{NC}$ interactions with an associated $\pi^{0}$. In Super-K, the current $\pi^{0}$ analysis is based on ring-finding satisfactory for proton decay signatures (pion momentum $0.5 \mathrm{GeV} / \mathrm{c}$ or lower). The efficiency for identifying two separate showers, using the current algorithms, is approximately $10 \%$ at $0.8 \mathrm{GeV}[43]$ and nearly zero at higher energies. This is mostly due to events with a small opening angle between the gamma showers,

${ }^{1}$ Estimated in round-numbers from the $\$ 100 \mathrm{M}$ cost of the Super-K. 


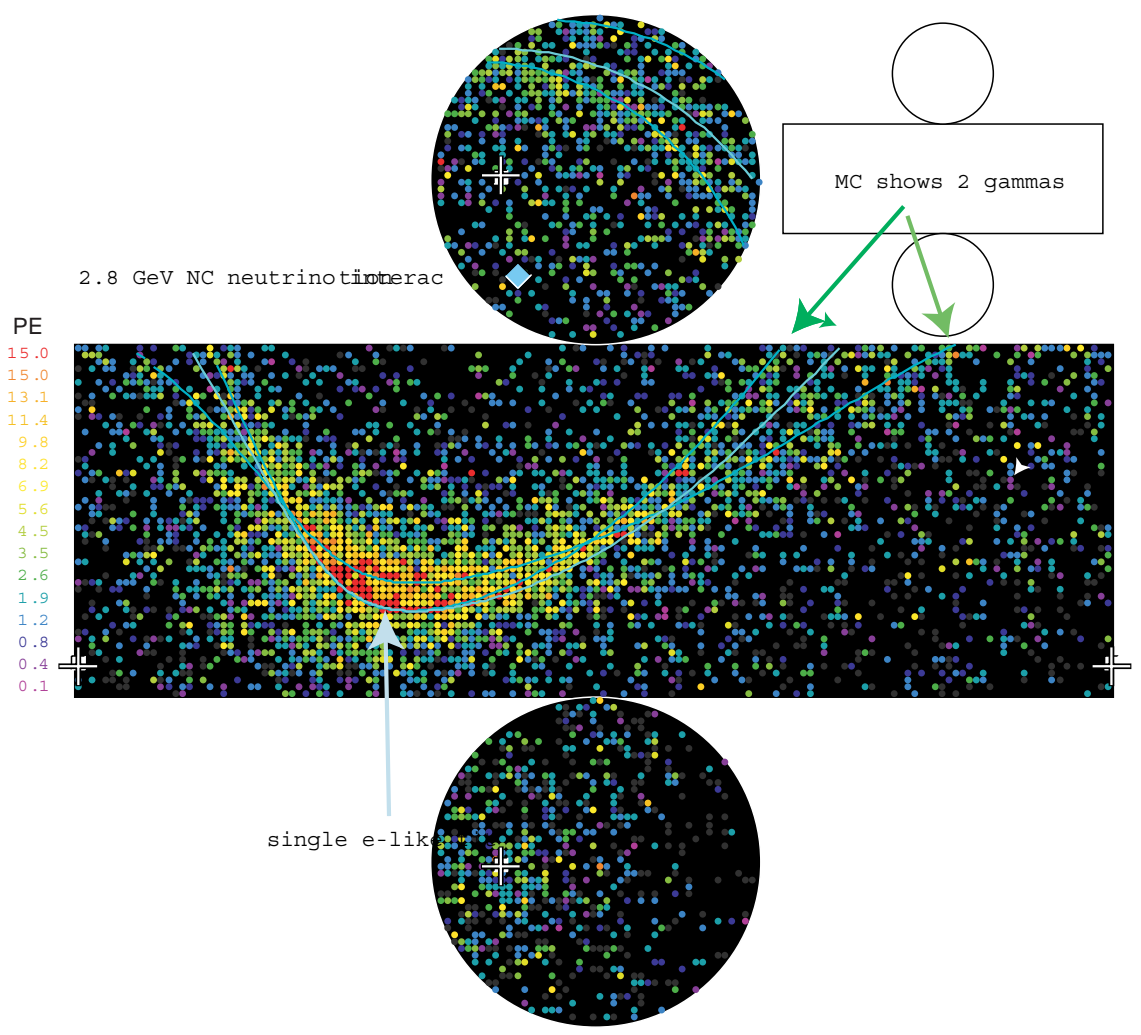

Figure A.14: An event display showing a typical neutral current $\pi^{0}$ event for the NuMI off-axis spectrum. The Super-K reconstruction software finds a single e-like ring.

typically 4 degrees for a $2 \mathrm{GeV} \pi^{0}$. Figure A.14 shows an event display of a simulated event in the Super-K detector. The event is a $2.5 \mathrm{GeV}$ neutral current $\pi^{0}$ where one Cherenkov ring was found. The Monte Carlo simulation shows two gamma rings, as does a reasonably trained human eye scan.

The problem in separating the two showers becomes more difficult up to about $0.8 \mathrm{GeV}$, but then should not get worse as one goes to higher energies. This is because the vertex separation becomes dominated by the photon attenuation length rather than the angle between the two decay gammas. Firstly, the mean of the angle $(\theta)$ between the two gammas is given by: 


$$
\overline{\cos \theta}=1-\frac{2}{\beta \gamma^{2}} \ln [\gamma(1+\beta)]
$$

At higher energies the second term becomes small, so that the mean separation angle goes as:

$$
\bar{\theta} \sim \frac{2}{\gamma} \sqrt{2 \gamma}=\frac{2 m_{\pi}}{E_{\pi}} \sqrt{2 \gamma}
$$

When the mean angle becomes less than the intrinsic detector resolution, angular separation becomes less important as a means to recognize $\pi^{0}$ 's from $e$ 's Figure A.15 shows the mean separation angle as a function of pion energy. This becomes equal to the Cherenkov light in water (dashed line) at about $0.6 \mathrm{GeV}$. At this energy, the rings from the shower begin to overlap and so algorithms must decide which PMT hit goes with which gamma. For a NuMI off-axis experiment, good separation to better than 10 degrees (dotted line) is required.

Until now, experiments have used standard TDC/ADC technology to record PMT data. It is clear that multi-hit waveform digitizers might prove to be very useful in better separating light from individual rings. This is because light from the two rings is likely to be separated in time even though overlapping in postion. Figure refF:gamdist shows the mean distance between the first interaction point of the two gammas. At energies below $1 \mathrm{GeV}$ this is influenced by the opening angle between the gammas so the difference is near a meter. At higher energies, however, the mean distance saturates due to the slow change of the photon attenuation length in water and so even near $3 \mathrm{GeV}$ the average distance is more than half a meter. This might be detectable with reasonably fast PMT's and standard waveform digitizers. Such studies are now in progress.

It is hopeful that improved and specialized reconstruction algorithms may have a significant impact on such high energy $\pi^{0}$ reconstruction. It is already the case that a specialized likelihood fitter has been employed to distinguish between single and double Cherenkov rings. This technique was employed in the studies for section 5.2 of the JHF neutrino proposal [20].

Using a prelimary version of a specialized $\pi^{0}$ fitter developed for Super-K, a study was performed of the hypothetical case of transplanting Super-K into the NuMI off-axis beam[42]. The standard Super-K atmospheric Monte Carlo was 


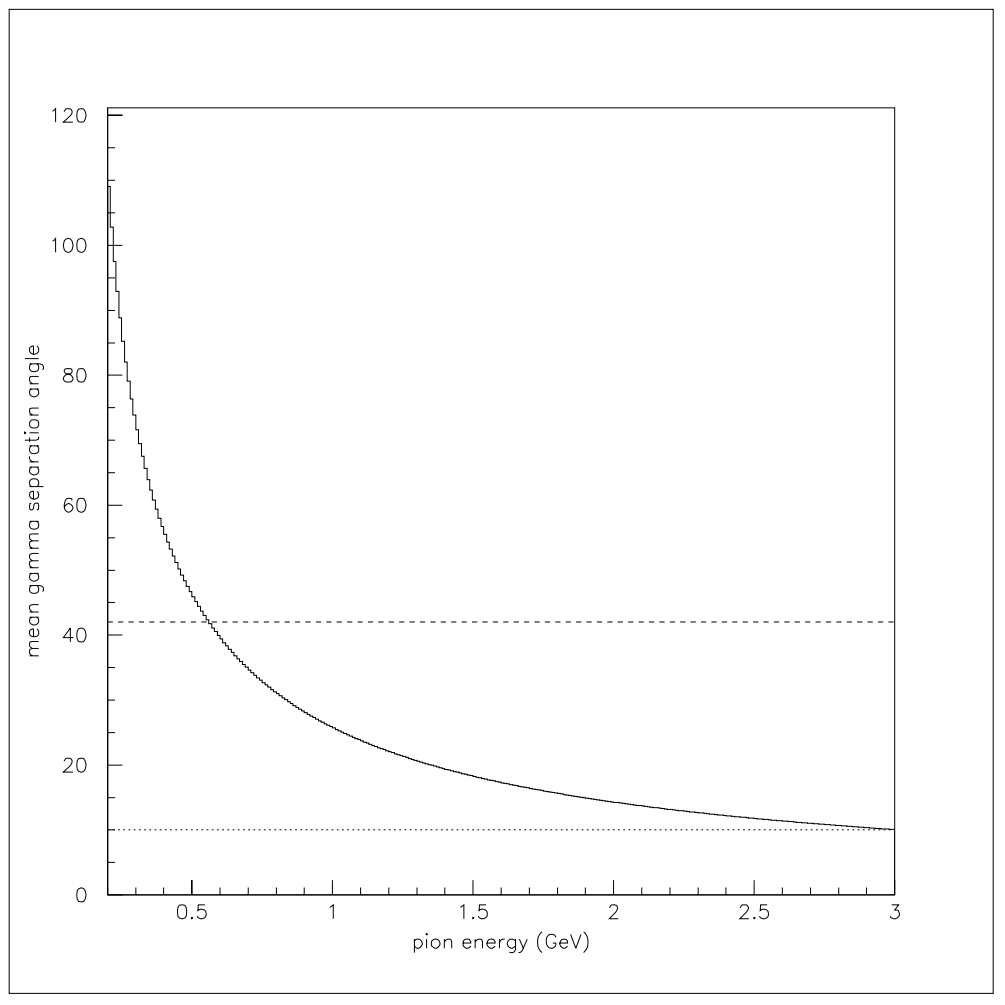

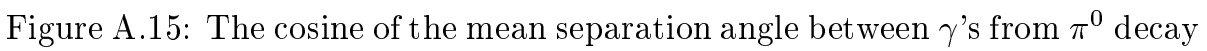
as a function of $E_{\pi}$.

used, reweighting each event to the NuMI beam spectrum using the Monte Carlo truth information. Nine event variables, such as decay electron tag, angle to the beam, the ratio of light in and outside of the single ring fit, and the value of the $\pi^{0}$ fit quality were combined into a likelihood to distinguish NC from $\nu_{e^{-}}$ $\mathrm{CC}$ interactions. The analysis was roughly $20-30 \%$ efficient for accepting $\nu_{e}-\mathrm{CC}$ signal, with a signal-to-background ratio of 0.7 -to- 1 .

There are further possibilities to be explored, including adding new transient recording electronics that can reconstruct multiple close in-time hits on a single PMT, to recover some events where one of the two gammas converts at a significantly displaced vertex.

With further study, the sensitivity of the analysis for $\nu_{e}$ appearance could be clarified using the comparably mature Monte Carlo and reconstruction software used for Super-K and IMB. At this time, it seems like a water Cherenkov is a difficult but but potentially viable detector for the off-axis experiment. 


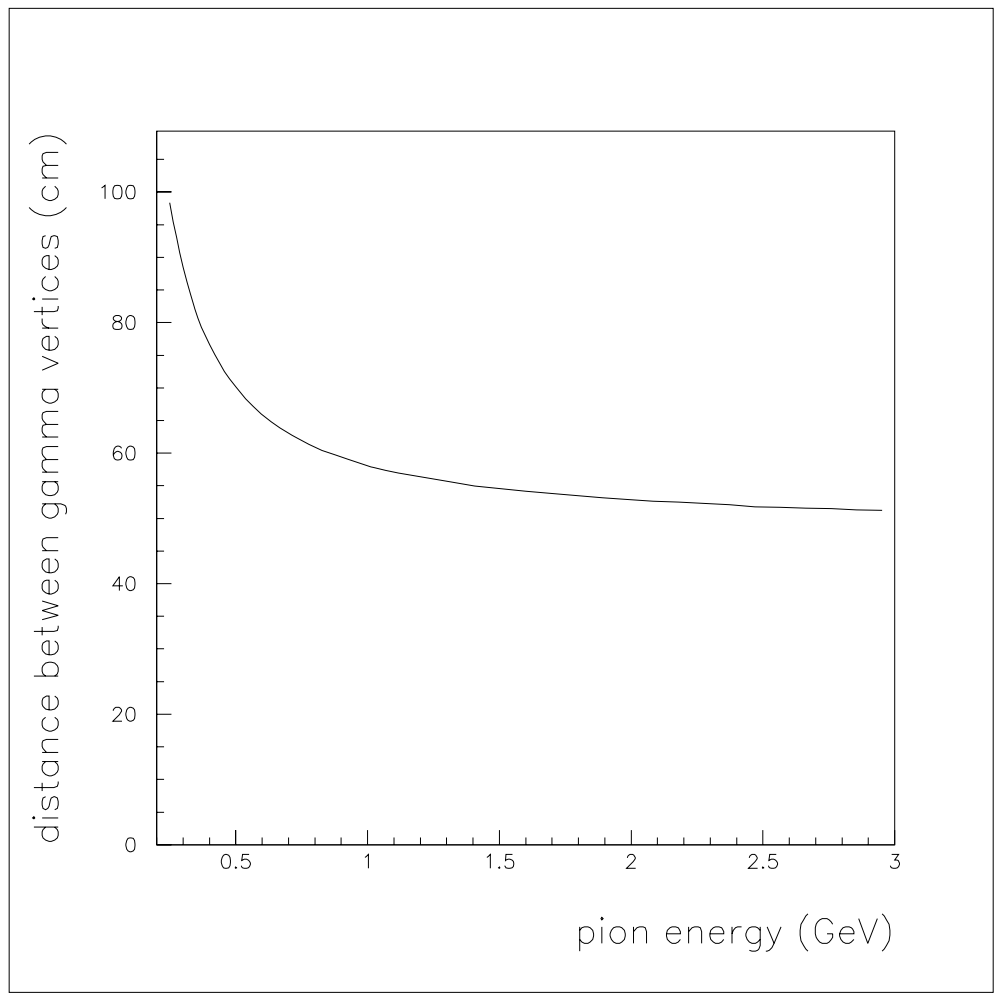

Figure A.16: The mean distance between the initial interaction point of the two $\gamma$ 's as a function of $E_{\pi}$.

There remains an interesting alternative path of inquiry. It is accepted that a Super-K like detector performs very well at lower energies (such as at 0.8 $\mathrm{GeV}$ at $\mathrm{K} 2 \mathrm{~K}$ and eventually the JHF). It may be interesting to consider moving such a detector even further off the NuMI axis, tuning the baseline and angle to second oscillation maximum. This would be at a lower peak energy, more suited to quasi-elastic kinematic reconstruction, and with considerably easier $\pi^{0}$ identification topologies. 


\section{A.3 Liquid Argon TPC}

The recent successful operation of the ICARUS 300-ton liquid-argon time-projectionchamber prototype $[44,45,46]$ indicates that it is timely to review the possibilities for large-scale application of this technology for accelerator-based neutrino physics, neutrino astrophysics, and proton decay [47, 48, 49].

A liquid argon detector for neutrino physics is a total absorption calorimeter with time-projection readout via the signal of drifting electrons collected in crossed planes of wires. The effective pixel size is about $5 \times 5 \times 5 \mathrm{~mm}^{3}$, compared to the radiation length of $14 \mathrm{~cm}$ and nuclear interaction length of $55 \mathrm{~cm}$. At a drift field strength of $500 \mathrm{~V} / \mathrm{cm}$, the drift velocity is about $1 \mathrm{~mm} / \mu \mathrm{s}$, so the drift time over, say, $5 \mathrm{~m}$ would be $5 \mathrm{~ms}$. Even if operated at the Earth's surface with no shielding, a liquid argon TPC has only about 1 (localized) cosmic-ray track per $\mathrm{m}^{2}$ of horizontal surface per drift time, so events appear very clean, as shown in Fig. A.17.

Because a liquid argon TPC is a total absorption detector with fine-grained sampling, it offers superior separation of charged-current and neutral-current neutrino interactions, as we as excellent identification of $e / \mu / \pi / K / p$, included $\pi^{0}$ 's. Hence, it is the most effective detector per unit mass for $\nu_{\mu} \rightarrow \nu_{e}$ appearance measurements (of $\sin ^{2} 2 \theta_{13}$, the sign of $\Delta m_{23}^{2}$, CP violation, ...), as shown in Fig. A.18.

To obtain economies of scale, a large liquid argon detector should be implemented in a single cryostat, such as those commonly used in the liquefied natural gas industry. Cryogenic volumes of up to $200,000 \mathrm{~m}^{3}$ (=280 kton if liquid argon) are now in use, as sketched in Fig. A.19.

An overall concept of a large magnetized liquid argon detector is shown in Fig. A.20. Anticipating the possibility that neutrino beams are eventually sent to it from more than one accelerator, the magnetic field is vertical so the trajectories of secondary particles are generally orthogonal to the magnetic field.

The usable drift distance in a liquid argon detector is limited by oxygen impurities to $2-5 \mathrm{~m}$. The diameter of a large liquid argon detector will be greater than (twice) the maximum drift distance, so the readout must consist of a set of parallel anode and cathode planes that subdivide the detector, as shown in Figs. A.20 and A.21.

A large liquid argon detector has excellent capability to search for nucleon decay [51], particularly because it is almost fully efficient for the decay $p \rightarrow K^{+} \bar{\nu}_{\mu}$ that is favored in many $\mathrm{SO}(10)$ SUSY models [52]. 


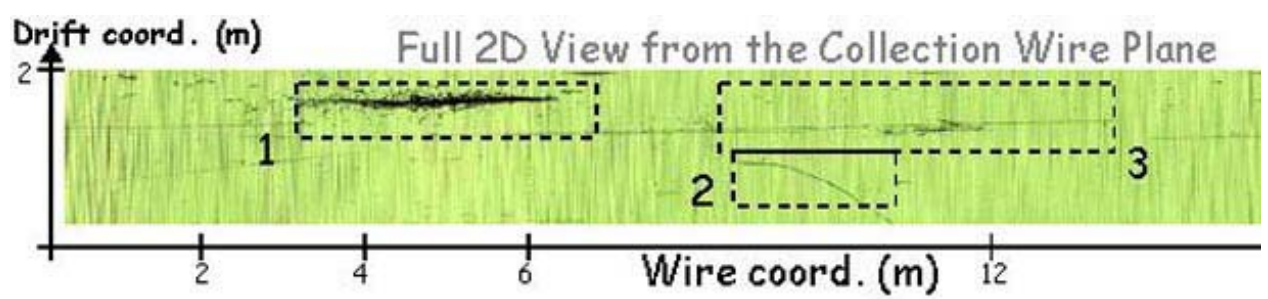

\section{Zoom details}

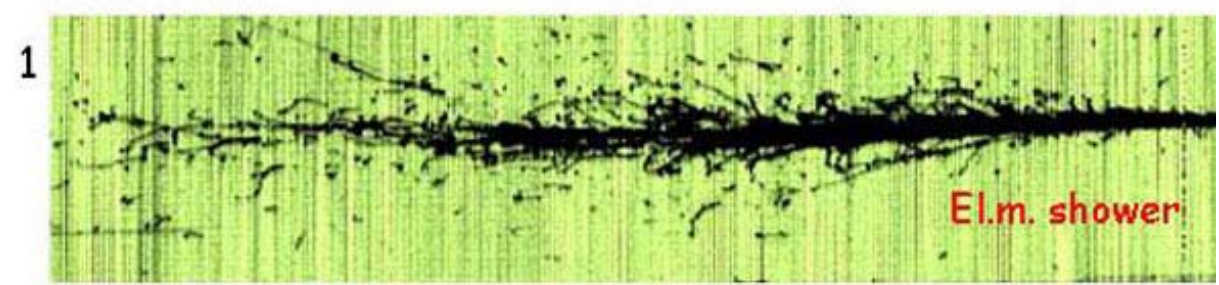

2
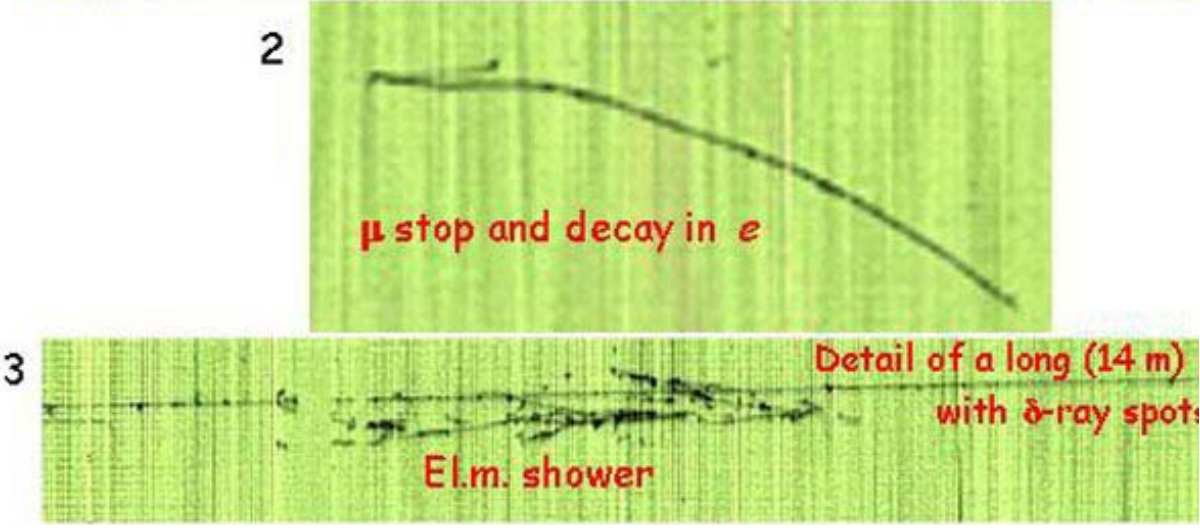

Figure A.17: An event from the recent cosmic-ray test run of ICARUS [45], showing excellent track resolution over long drift distances in zero magnetic field.

The data acquisition for a liquid-argon TPC can operate in a pipelined, deadtimeless mode, with zero-suppression [53]. This could permit fully live operation at the Earth's surface for nucleon decay studies, in addition to triggered data collection of neutrino interactions from a pulsed accelerator beam. The data rate would, of course, be quite high in this case. It may nonetheless be less costly to implement a high rate data-acquisition system than to reduce the un-triggered data rate by siting the detector deep underground.

In the future, neutrino beams from pion decay may be supplanted by those from muon decay [54], in which cases beams of $\nu_{\mu}$ and $\bar{\nu}_{e}$ or $\bar{\nu}_{\mu}$ and $\nu_{e}$ would be available. To take advantage of such beams for studies of $\mathrm{CP}$ violation, the 


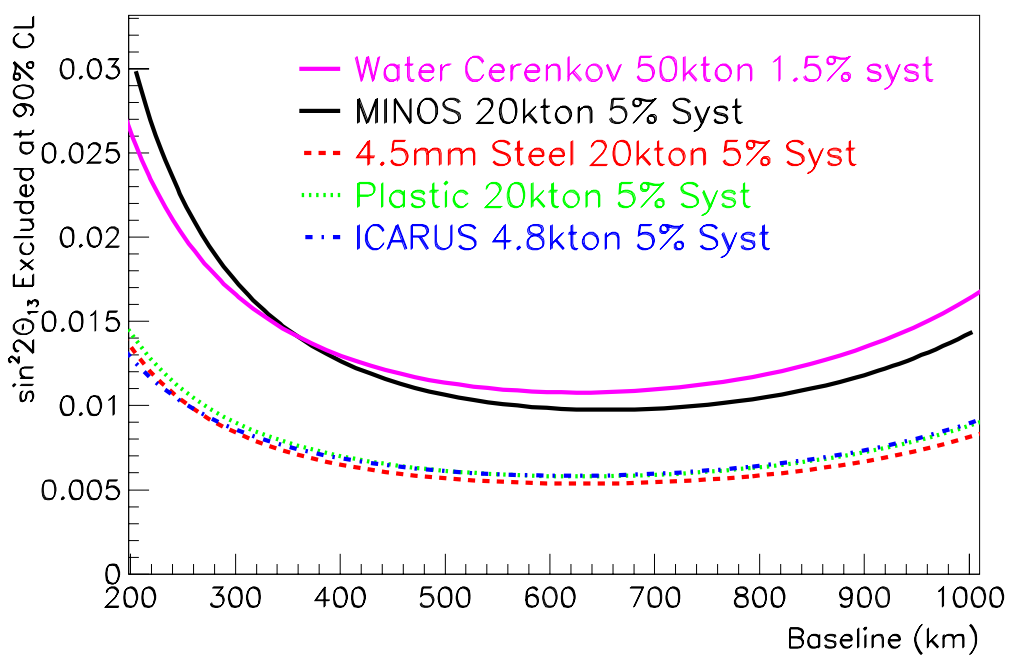

Figure A.18: Comparison of several types of detectors in measuring $\sin ^{2} 2 \theta_{13}$ in the presence of backgrounds typical of a pion-decay neutrino beam at intermediate baselines [50]. The detector labeled ICARUS [45] is a liquid argon time projection chamber. With a 25-kton liquid argon detector, and an off-axis NuMI neutrino beam, the sensitivity to $\sin ^{2} 2 \theta_{13}$ would be at least 0.002 .

sign of the final state lepton much be determined in the detector, which must therefore be immersed in a magnetic field. A liquid argon TPC is compatible with a superimposed magnetic field. The sign of muons could be well determined with a field of only $0.1 \mathrm{~T}$, and the sign of electrons up to a few $\mathrm{GeV}$ energy could be determined in a field of $0.5 \mathrm{~T}$ by analysis of the curvature of tracks in the electromagnetic shower $[55,56]$.

As well as being the highest-performance large detector for neutrinos, a liquid argon TPC is also one of the least costly. Liquid argon costs about $\$ 1 \mathrm{M}$ per kton, or $\$ 20 \mathrm{~m}$ for a 20 kton detector. Such a detector would require about $100 \mathrm{k}$ readout channels, at a cost of about $\$ 10 \mathrm{M}$. The greatest cost uncertainty at present is in the storage tank and associated cryogenic/purification system. A initial engineering study on this issue is underway [57]. If the cryo system cost proves to be of order the cost of the argon itself, then a 20 kton detector would cost about $\$ 50 \mathrm{M}$ - which would make a liquid argon TPC the price/performance leader for large neutrino detectors. 


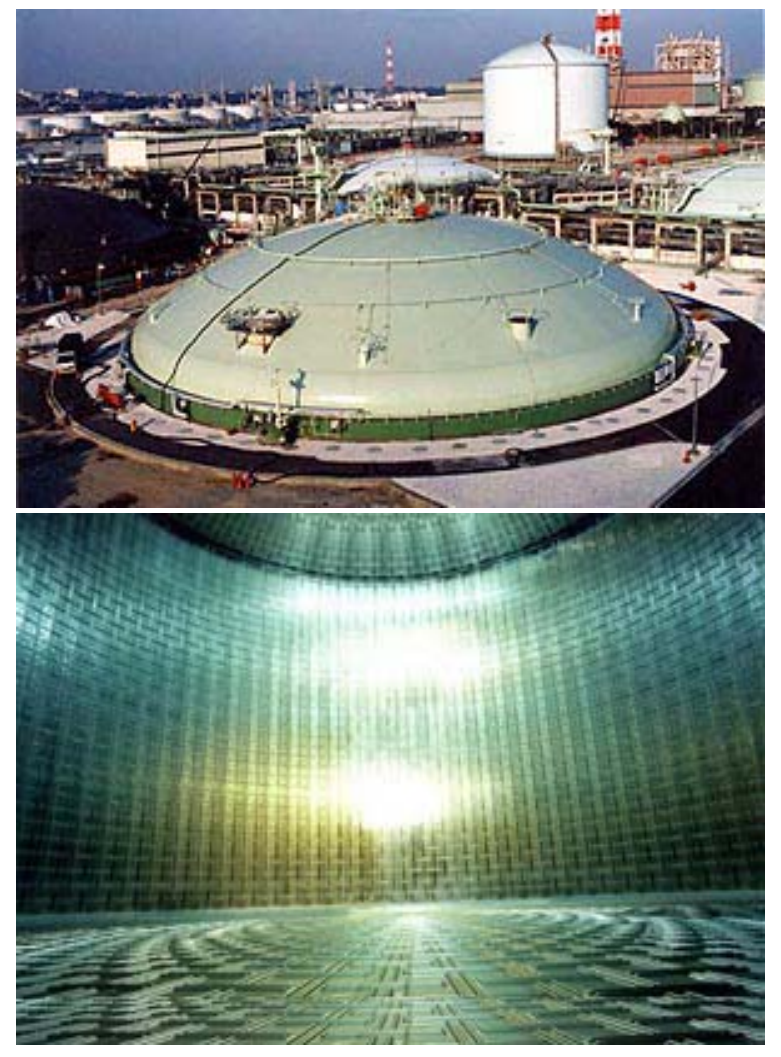

Figure A.19: Photographs of cryogenic storage tanks of volumes $\approx 100,000 \mathrm{~m}^{3}$.

A-36 


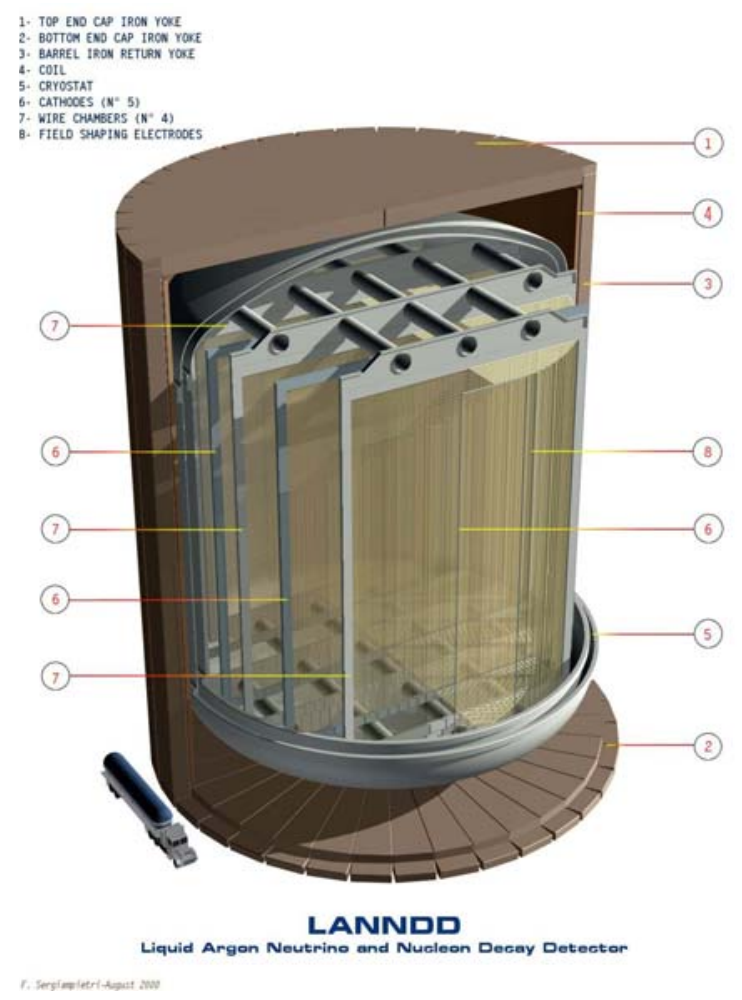

Figure A.20: Concept of a 70-kton Liquid Argon Neutrino and Nucleon Decay Detector (LANNDD) [47, 48]. 


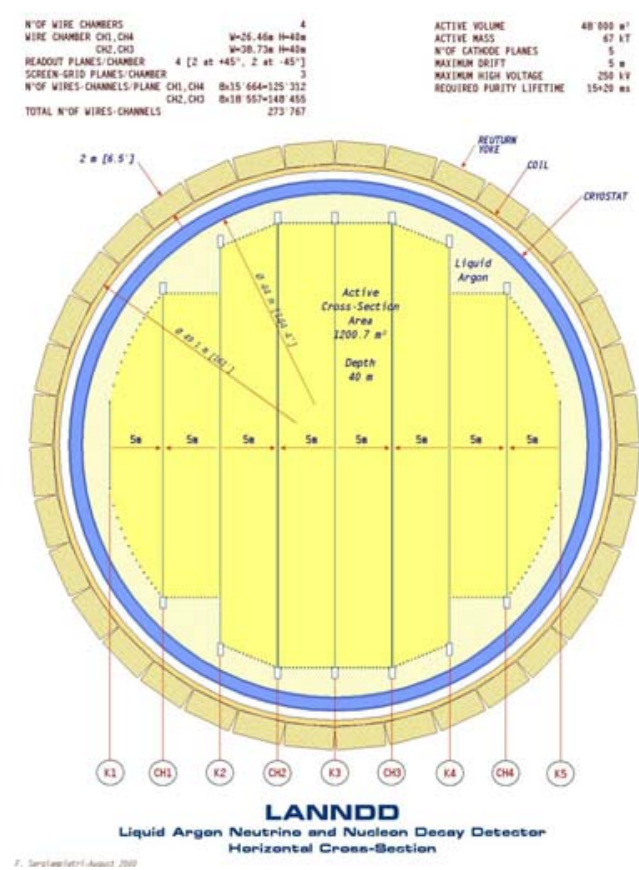

Figure A.21: Top view of the electrode arrangement of a 70-kton Liquid Argon Neutrino and Nucleon Decay Detector (LANNDD) [48].

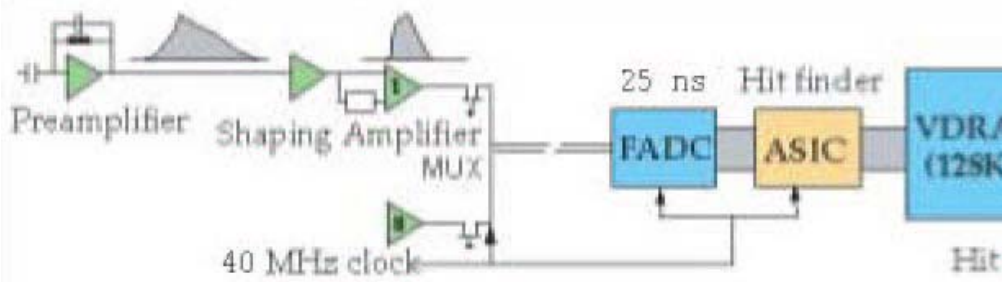

Figure A.22: Scheme of the front-end electronics of the ICARUS detector [53]. 


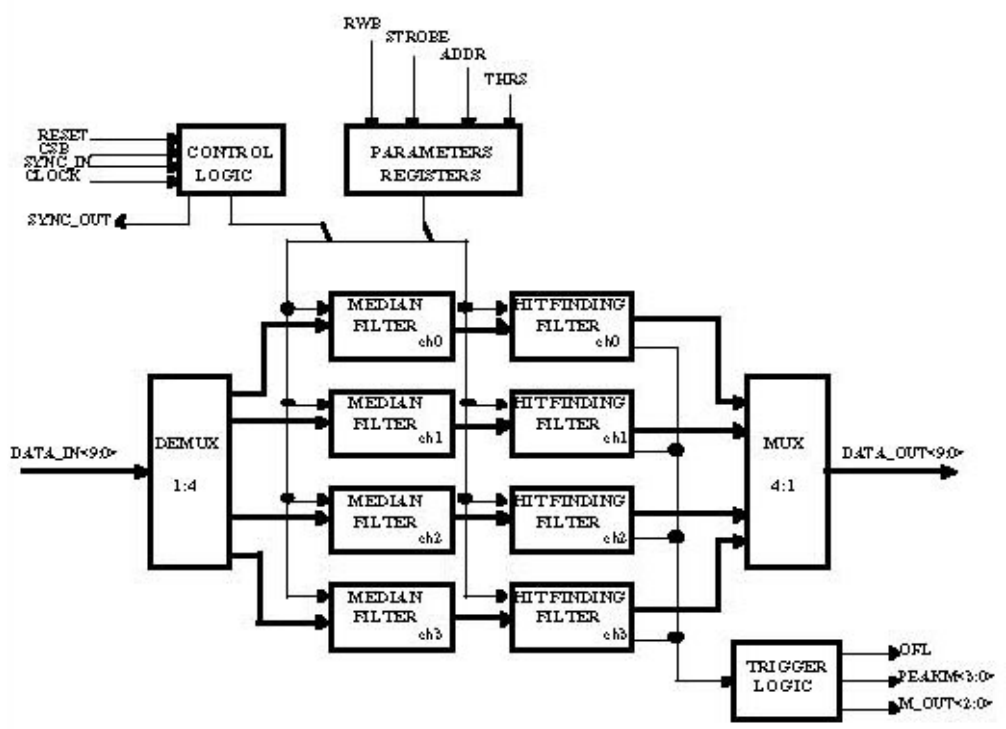

Figure A.23: Scheme of the back-end electronics of the ICARUS detector [53].

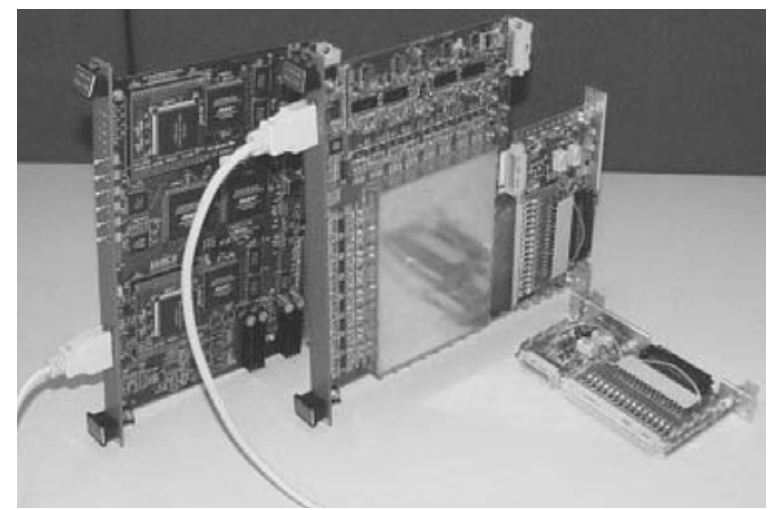

Figure A.24: Readout electronics of the ICARUS detector [53]. 


\section{Appendix B}

\section{Possible Sites for a NuMI Off-Axis Detector}

\section{B.1 Criteria for Site Selection}

There appears to be general agreement that because of the $10 \mu \mathrm{s}$ beam spill, a NuMI off-axis detector can be located in a surface or near-surface laboratory. Besides the general physics considerations discussed in the preceeding section there are more specific requirements that determine whether a give site is adequate. The most important ones are listed below.

a) The site should be at least $450 \mathrm{~m}$ along an axis pointing towards Fermilab and $100 \mathrm{~m}$ along the transverse access. Such a site will accommodate the largest possible detector, earth berms for shielding, if they are required, and space for ancillary facilities. Access to the entire site should be protectable by lease or options, even though only a portion of the site is necessary for the first stage laboratory.

b) The site should permit cost-effective construction of the detector and associated structures, if any. The detector may need shielding and good availability of material for shielding is an advantage.

c) The site should have good highway access. A location close to Soudan is not a requirement. However, a site close enough to Soudan to enable easy staff travel between the laboratories would facilitate laboratory management and result in savings in operating costs. 
d) The site should have good access to $\sim 1 \mathrm{MW}$ of electrical power and telecommunications, including fiber optic data links. The site needs access to water in quantities that depend on detector design. Existing sewage treatment access would be a plus for any site.

e) An ideal site would have low environmental and political risk, that is, not adjacent to large populations, to conservation or park areas or to areas of natural beauty, such as shorelines or other features. An ideal site would also have soil and rock with low content of sulfur and heavy metals, in order to minimize environmental issues due to leaching.

f) Depending on future information about information about $\Delta m^{2}$, matter effects or CP violation, the detector may be moved at some future time. Although such a relocation might need to be far, in order to substantially change the baseline, another possibility is a short move transverse to the beam. Thus, a site near other ideal sites located at different transverse dimensions from the beam center line would have some advantage.

g) An ideal site would have gravity drainage, even for an underground laboratory location. Such drainage reduces both operating cost and risk of flooding due to electrical or mechanical failure.

\section{B.2 Potential Sites}

We have initial examination of possible sites which might satisfy the above requirements. So far, only one has been sufficiently studied. This site, at $712 \mathrm{~km}$ from Fermilab is a former mining site owned by LTV. Preliminary discussions with the appropriate parties lead us to believe that there would be a positive response to our potential request to site the detector there. The principal negative factor is that the site is not as far away from Fermilab as one would desire ideally. It is probably adequate if $\Delta m_{32}^{2}$ is above $2.5 \times 10^{-3}$. The optimal energy for $\Delta m_{32}^{2}=2.5 \times 10^{-3}$ would be somewhat below $1.5 \mathrm{GeV}$. Such a low energy might make the rejection of $\mathrm{NC}$ background somewhat easier. The site is described in more detail in the Appendix B.

From examination of the map of Ontario, there are two potential site areas further north, in Canada. The first one lies along the southern Trans Canadian Highway, and is about $850 \mathrm{~km}$ away from Fermilab. A somewhat more distant variant in this site area would be along Rt 502 which runs north by north east from the main highway and could offer a site about $20-40 \mathrm{~km}$ further. The other site area would be at about $985 \mathrm{~km}$, paralleling the northern branch of the Trans-Canadian Highway. There are also two railroad tracks along the highway, one just south of it and the other one about $20 \mathrm{~km}$ north of it. This site would be a little too far if $\Delta m_{32}^{2}$ is above $2.5 \times 10^{-3} \mathrm{eV}^{2}$. At this time we do not 


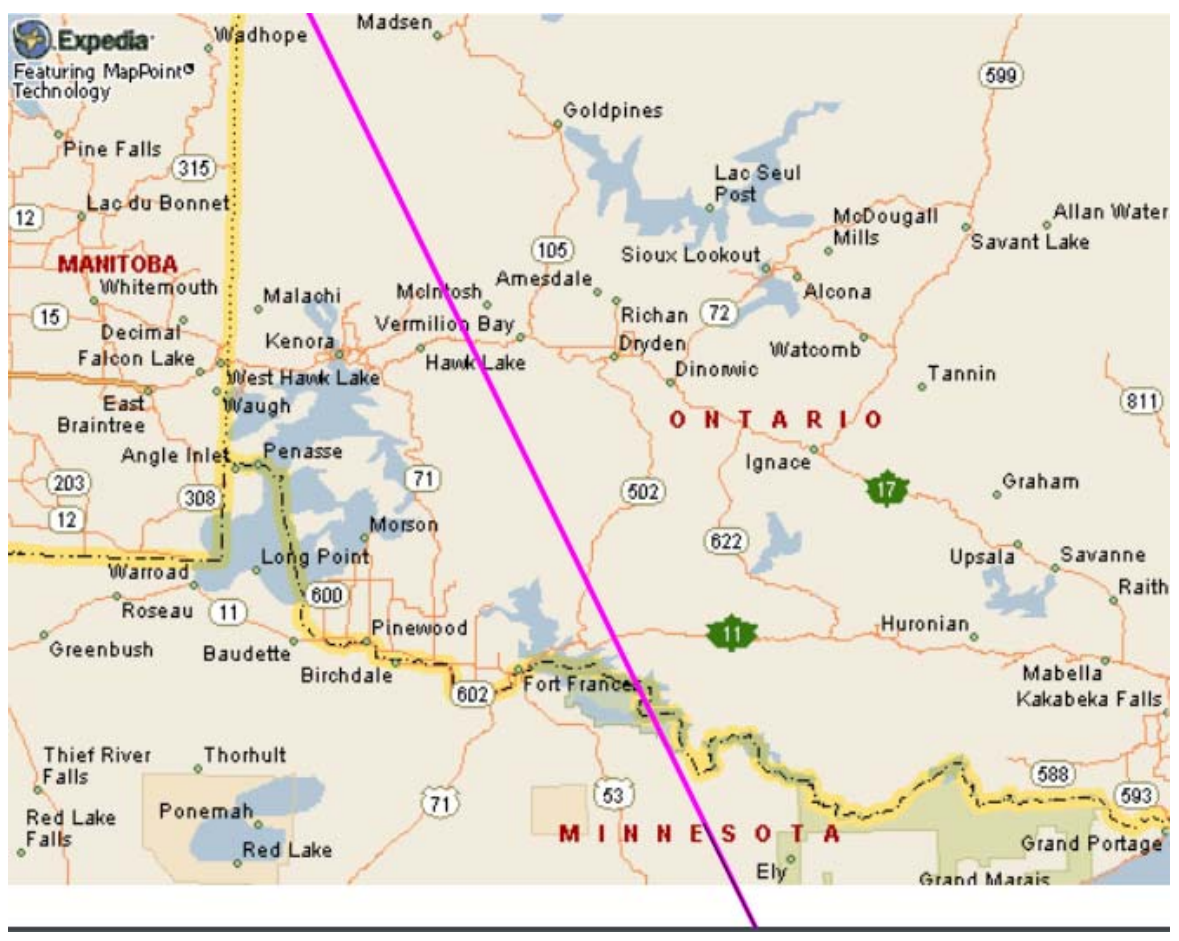

Figure B.1: Map of southern Ontario and the NuMI beam direction

know whether it would be possible to operate a neutrino detector in either one of these areas.

Looking further into the future one might look at possible sites for Phase II, perhaps at the $L / E$ corresponding to the second oscillation maximum. This would involve going further north, to a distance of about $1100 \mathrm{~km}$ and energy around $1 \mathrm{GeV}$. A possible site is in the neighborhood of Red Lake. This is a pretty desolate area and on-site investigation will be required to see if it could be suitable. A map of this area of Canada, with the 0 deg beam line superimposed, is shown in Fig. B.1. A list of the potential sites for an off-axis experiment is shown in Table B.2.

\section{B.3 LTV sites}

While the investigation of possible sites continues, the sites for which we have the most information already more than meet the minimum criteria for an ac- 


\begin{tabular}{|c|c|c|c|c|}
\hline Area & $\begin{array}{c}\text { Distance } \\
\text { to Fermilab }\end{array}$ & Depth & Access & Comments \\
\hline Iron River & $612 \mathrm{~km}$ & $6.5 \mathrm{~km}$ & $\begin{array}{c}\text { Wisconsin } \\
\text { Highway } 13\end{array}$ & $\begin{array}{c}\text { Near mouth of } \\
\text { Iron River; } \\
\text { rural, forest }\end{array}$ \\
\hline $\begin{array}{l}\text { Two Harbors } \\
\text { (Lake Superior } \\
\text { North Shore) }\end{array}$ & $643 \mathrm{~km}$ & $5.2 \mathrm{~km}$ & $\begin{array}{c}\text { U.S. Highway } \\
61\end{array}$ & $\begin{array}{l}\text { Two Harbors } \\
\text { MN; mixed } \\
\text { urban and rural }\end{array}$ \\
\hline Tower-Soudan-Embarass & 720 to $735 \mathrm{~km}$ & $1 \mathrm{~km}$ & $\begin{array}{c}\text { MN Highway } \\
169\end{array}$ & $\begin{array}{c}\text { Off-axis } \\
\text { locations rural, } \\
\text { forest }\end{array}$ \\
\hline Buyck & $775 \mathrm{~km}$ & $-1.8 \mathrm{~km}$ & $\begin{array}{c}\text { Orr-Buyck-Ely } \\
\text { Road (Echo } \\
\text { Trail) }\end{array}$ & $\begin{array}{c}\text { Off-axis } \\
\text { locations rural, } \\
\text { forest }\end{array}$ \\
\hline Ash River & $\sim 800 \mathrm{~km}$ & $-3.4 \mathrm{~km}$ & $\begin{array}{l}\text { Ash River Trail, } \\
\text { east of U.S. } 53\end{array}$ & $\begin{array}{c}\text { Kabetogama } \\
\text { State Forest } \\
\text { south side of } \\
\text { Voyageur's } \\
\text { National Park }\end{array}$ \\
\hline Farrington & $\sim 850 \mathrm{~km}$ & $-6.9 \mathrm{~km}$ & $\begin{array}{c}\text { Ontario } \\
\text { Highway } 11 \\
\text { east of Fort } \\
\text { Frances }\end{array}$ & \\
\hline Otukamamoan Lake & $\sim 900 \mathrm{~km}$ & $-10.9 \mathrm{~km}$ & $\begin{array}{c}\text { Ontario } \\
\text { Highway } 502 \\
\text { northeast of Fort } \\
\text { Frances }\end{array}$ & \\
\hline Vermilion Bay & $\sim 950 \mathrm{~km}$ & $-15.3 \mathrm{~km}$ & $\begin{array}{c}\text { Ontario } \\
\text { Highway } 17 \\
\text { east of Kenora, } \\
\text { west of Dryden }\end{array}$ & \\
\hline
\end{tabular}

Table B.1: Possible sites for an off-axis experiment

ceptable site. In particular, we have identified two sites that lie in Minnesota's Iron Range, a mixed hematite, magnetite and taconite deposit several kilometers in width that extends $\sim 200 \mathrm{~km}$ ENE from Grand Rapids MN to Babbitt MN. These two sites are just north of Erie Pit No. 1 in the former LTV Steel Mine about $10 \mathrm{~km}$ north of Aurora MN. Both sites consist of tailings piles, each $\sim 15-20 \mathrm{~m}$ in height, $500 \mathrm{~m}$ in the beam direction and $>200 \mathrm{~m}$ transverse to the beam direction. The westerly of the two sites is $10.3 \mathrm{~km}$ from the beam axis; the easterly site is $9.1 \mathrm{~km}$ from the beam axis. Both sites are $\sim 712 \mathrm{~km}$ from Fermilab. These sites are currently owned by Cliffs-Erie LLC, an iron mining company. There is neither active mining nor plans for mining within several kilometers of either site. Both sites are accessible by a private two-lane paved highway from MN Highway 135. The sites are located $\sim 20$ miles by state highway from Soudan.

Both LTV sites have good access to mine electrical power. An existing power line connects the two sites. Public utility power is available along Highway 135 and it 
may be possible to reconfigure the existing line to provide public utility power to either site. Fiber optic telecommunications exist along Highway 135 and connect to cell phone towers located on the peaks of the Embarrass Mountains less than $2 \mathrm{~km}$ north and west of the LTV sites. Erie Pit No. 1, which is about $6 \mathrm{~km}$ in length, is a potential source of water for a detector and also provides natural drainage for both sites. The LTV Mine is $\sim 20 \mathrm{~km}$ transverse to the beam line. It extends past the beam centerline to the east and about $5 \mathrm{~km}$ to the west of these two sites. Numerous tailings piles can be found on the LTV site if moving the detector becomes desirable at some later time. Tailings piles also exist further west along Highway 135, both east and west of Biwabik MN. Biwabik is $\sim 20 \mathrm{~km}$ west of the NuMI beam centerline.

Preliminary information from Michael Johnson, the site manager for Cliffs-Erie indicates low levels of sulfur and heavy metals at these sites. (The other end of the LTV property, east of the beam centerline is believed to have coppernickel-precious metal deposits in a sulfide ore, with new mining activity possible during the next several years.) Thus, we believe from current information the environmental risk associated with this property is low. Because of previous mining activity, the political risk is also low. Indeed, the IRRRB, the regional state economic development agency, has already indicated positive political and possible financial support for these sites. The positive reputation of the Soudan Lab is also strong in this area, which also minimizes political risk. Mr. Johnson on behalf of Cliffs-Erie has indicated a willingness of the company to discuss various acquisition options any time we are ready.

In summary, the LTV sites located 9.1 and $10.3 \mathrm{~km}$ transverse to the beam centerline, $712 \mathrm{~km}$ from Fermilab provide high-quality, low-risk areas for an off-axis detector, which could be accessed in a short period of time. 